\title{
Extragradient method for convex minimization problem
}

\author{
Lu-Chuan Ceng ${ }^{1,2}$, Yeong-Cheng Liou ${ }^{3,4^{*}}$ and Ching-Feng Wen ${ }^{4}$
}

\author{
"Correspondence: \\ simplex_liou@hotmail.com \\ ${ }^{3}$ Department of Information \\ Management, Cheng Shiu \\ University, Kaohsiung, 833, Taiwan \\ ${ }^{4}$ Center for Fundamental Science, \\ Kaohsiung Medical University, \\ Kaohsiung, 807, Taiwan \\ Full list of author information is \\ available at the end of the article
}

\begin{abstract}
In this paper, we introduce and analyze a multi-step hybrid extragradient algorithm by combining Korpelevich's extragradient method, the viscosity approximation method, the hybrid steepest-descent method, Mann's iteration method and the gradient-projection method (GPM) with regularization in the setting of infinite-dimensional Hilbert spaces. It is proven that, under appropriate assumptions, the proposed algorithm converges strongly to a solution of the convex minimization problem (CMP) with constraints of several problems: finitely many generalized mixed equilibrium problems (GMEPs), finitely many variational inclusions, and the fixed point problem of a strictly pseudocontractive mapping. In the meantime, we also prove the strong convergence of the proposed algorithm to the unique solution of a hierarchical variational inequality problem (over the fixed point set of a strictly pseudocontractive mapping) with constraints of finitely many GMEPs, finitely many variational inclusions and the CMP. The results presented in this paper improve and extend the corresponding results announced by many others.
\end{abstract}

MSC: 49J30; 47H09; 47J20; 49M05

Keywords: hybrid extragradient approach; split feasibility problem; generalized mixed equilibrium problem; variational inclusion; strictly pseudocontractive mapping; nonexpansive mapping

\section{Introduction}

Let $C$ be a nonempty closed convex subset of a real Hilbert space $H$ and $P_{C}$ be the metric projection of $H$ onto $C$. Let $S: C \rightarrow H$ be a nonlinear mapping on $C$. We denote by Fix $(S)$ the set of fixed points of $S$ and by $\mathbf{R}$ the set of all real numbers. A mapping $S: C \rightarrow H$ is called $L$-Lipschitz-continuous (or $L$-Lipschitzian) if there exists a constant $L \geq 0$ such that

$$
\|S x-S y\| \leq L\|x-y\|, \quad \forall x, y \in C .
$$

In particular, if $L=1$ then $S$ is called a nonexpansive mapping; if $L \in[0,1)$ then $S$ is called a contraction.

A mapping $A: C \rightarrow H$ is said to be $\zeta$-inverse-strongly monotone if there exists $\zeta>0$ such that

$$
\langle A x-A y, x-y\rangle \geq \zeta\|A x-A y\|^{2}, \quad \forall x, y \in C .
$$

O2014 Ceng et al.; licensee Springer. This is an Open Access article distributed under the terms of the Creative Commons Attribution License (http://creativecommons.org/licenses/by/2.0), which permits unrestricted use, distribution, and reproduction in any medium, provided the original work is properly cited. 
It is clear that every inverse-strongly monotone mapping is a monotone and Lipschitzcontinuous mapping. A mapping $T: C \rightarrow C$ is said to be $\xi$-strictly pseudocontractive if there exists $\xi \in[0,1)$ such that

$$
\|T x-T y\|^{2} \leq\|x-y\|^{2}+\xi\|(I-T) x-(I-T) y\|^{2}, \quad \forall x, y \in C .
$$

In this case, we also say that $T$ is a $\xi$-strict pseudocontraction. In particular, whenever $\xi=0, T$ becomes a nonexpansive mapping from $C$ into itself.

Let $A: C \rightarrow H$ be a nonlinear mapping on $C$. We consider the following variational inequality problem (VIP): find a point $x \in C$ such that

$$
\langle A x, y-x\rangle \geq 0, \quad \forall y \in C .
$$

The solution set of $\operatorname{VIP}(1.1)$ is denoted by $\operatorname{VI}(C, A)$.

The VIP (1.1) was first discussed by Lions [1]. There are many applications of VIP (1.1) in various fields; see e.g., [2-5]. It is well known that, if $A$ is a strongly monotone and Lipschitz-continuous mapping on $C$, then VIP (1.1) has a unique solution. In 1976, Korpelevich [6] proposed an iterative algorithm for solving the VIP (1.1) in Euclidean space $\mathbf{R}^{n}$ :

$$
\left\{\begin{array}{l}
y_{n}=P_{C}\left(x_{n}-\tau A x_{n}\right), \\
x_{n+1}=P_{C}\left(x_{n}-\tau A y_{n}\right), \quad \forall n \geq 0,
\end{array}\right.
$$

with $\tau>0$ a given number, which is known as the extragradient method. The literature on the VIP is vast and Korpelevich's extragradient method has received great attention given by many authors, who improved it in various ways; see e.g., [7-19] and references therein, to name but a few.

Consider the following constrained convex minimization problem (CMP):

$$
\operatorname{minimize}\{f(x): x \in C\}
$$

where $f: C \rightarrow \mathbf{R}$ is a real-valued convex functional. We denote by $\Gamma$ the solution set of the CMP (1.2). If $f$ is Fréchet differentiable, then the gradient-projection method (GPM) generates a sequence $\left\{x_{n}\right\}$ via the recursive formula

$$
x_{n+1}=P_{C}\left(x_{n}-\lambda \nabla f\left(x_{n}\right)\right), \quad \forall n \geq 0,
$$

or more generally,

$$
x_{n+1}=P_{C}\left(x_{n}-\lambda_{n} \nabla f\left(x_{n}\right)\right), \quad \forall n \geq 0,
$$

where in both (1.3) and (1.4), the initial guess $x_{0}$ is taken from $C$ arbitrarily, the parameters, $\lambda$ or $\lambda_{n}$, are positive real numbers, and $P_{C}$ is the metric projection from $H$ onto $C$. The convergence of the algorithms (1.3) and (1.4) depends on the behavior of the gradient $\nabla f$. As a matter of fact, it is well known that if $\nabla f$ is strongly monotone and Lipschitzian; namely, there are constants $\eta, L>0$ satisfying the properties

$$
\langle\nabla f(x)-\nabla f(y), x-y\rangle \geq \eta\|x-y\|^{2} \quad \text { and } \quad\|\nabla f(x)-\nabla f(y)\| \leq L\|x-y\|, \quad \forall x, y \in C,
$$


then, for $0<\lambda<2 \eta / L^{2}$, the operator $T:=P_{C}(I-\lambda \nabla f)$ is a contraction; hence, the sequence $\left\{x_{n}\right\}$ defined by (1.3) converges in norm to the unique solution of the CMP (1.2). More generally, if the sequence $\left\{\lambda_{n}\right\}$ is chosen to satisfy the property $0<\liminf _{n \rightarrow \infty} \lambda_{n} \leq$ $\lim \sup _{n \rightarrow \infty} \lambda_{n}<2 \eta / L^{2}$, then the sequence $\left\{x_{n}\right\}$ defined by (1.4) converges in norm to the unique minimizer of the CMP (1.2). However, if the gradient $\nabla f$ fails to be strongly monotone, the operator $T$ defined by $T:=P_{C}(I-\lambda \nabla f)$ would fail to be contractive; consequently, the sequence $\left\{x_{n}\right\}$ generated by (1.3) may fail to converge strongly (see Section 4 in $\mathrm{Xu}[20])$.

Theorem 1.1 (see [20, Theorem 5.2]) Assume the CMP (1.2) is consistent and let $\Gamma$ denote its solution set. Assume the gradient $\nabla f$ satisfies the Lipschitz condition with constant $L>0$. Let $V: C \rightarrow C$ be a $\rho$-contraction with coefficient $\rho \in[0,1)$. Let a sequence $\left\{x_{n}\right\}$ be generated by the following hybrid gradient-projection algorithm (GPA):

$$
x_{n+1}=\alpha_{n} V x_{n}+\left(1-\alpha_{n}\right) P_{C}\left(x_{n}-\lambda_{n} \nabla f\left(x_{n}\right)\right), \quad \forall n \geq 0 .
$$

Assume the sequence $\left\{\lambda_{n}\right\}$ satisfies the condition $0<\liminf _{n \rightarrow \infty} \lambda_{n} \leq \lim \sup _{n \rightarrow \infty} \lambda_{n}<2 / L$, and, in addition, the following conditions are satisfied for $\left\{\lambda_{n}\right\}$ and $\left\{\alpha_{n}\right\} \subset[0,1]:$ (i) $\alpha_{n} \rightarrow$ 0 , (ii) $\sum_{n=0}^{\infty} \alpha_{n}=\infty$, (iii) $\sum_{n=0}^{\infty}\left|\alpha_{n+1}-\alpha_{n}\right|<\infty$, and (iv) $\sum_{n=0}^{\infty}\left|\lambda_{n+1}-\lambda_{n}\right|<\infty$. Then the sequence $\left\{x_{n}\right\}$ converges in norm to a minimizer of CMP (1.2), which is also the unique solution $x^{*} \in \Gamma$ to the VIP

$$
\left\langle(I-V) x^{*}, x-x^{*}\right\rangle \geq 0, \quad \forall x \in \Gamma .
$$

In other words, $x^{*}$ is the unique fixed point of the contraction $P_{\Gamma} V, x^{*}=P_{\Gamma} V x^{*}$.

On the other hand, let $S$ and $T$ be two nonexpansive mappings. In 2009, Yao et al. [21] considered the following hierarchical variational inequality problem (HVIP): find hierarchically a fixed point of $T$, which is a solution to the VIP for monotone mapping $I-S$; namely, find $\tilde{x} \in \operatorname{Fix}(T)$ such that

$$
\langle(I-S) \tilde{x}, p-\tilde{x}\rangle \geq 0, \quad \forall p \in \operatorname{Fix}(T) .
$$

The solution set of the hierarchical VIP (1.7) is denoted by $\Lambda$. It is not hard to check that solving the hierarchical VIP (1.7) is equivalent to the fixed point problem of the composite mapping $P_{\mathrm{Fix}(T)} S$, i.e., find $\tilde{x} \in C$ such that $\tilde{x}=P_{\mathrm{Fix}(T)} S \tilde{x}$. The authors [21] introduced and analyzed the following iterative algorithm for solving the HVIP (1.7):

$$
\left\{\begin{array}{l}
y_{n}=\beta_{n} S x_{n}+\left(1-\beta_{n}\right) x_{n}, \\
x_{n+1}=\alpha_{n} V x_{n}+\left(1-\alpha_{n}\right) T y_{n}, \quad \forall n \geq 0 .
\end{array}\right.
$$

Theorem 1.2 (see [21, Theorem 3.2]) Let $C$ be a nonempty closed convex subset of a real Hilbert space H. Let $S$ and $T$ be two nonexpansive mappings of $C$ into itself. Let $V: C \rightarrow C$ be a fixed contraction with $\alpha \in(0,1)$. Let $\left\{\alpha_{n}\right\}$ and $\left\{\beta_{n}\right\}$ be two sequences in $(0,1)$. For any given $x_{0} \in C$, let $\left\{x_{n}\right\}$ be the sequence generated by (1.8). Assume that the sequence $\left\{x_{n}\right\}$ is bounded and that

(i) $\sum_{n=0}^{\infty} \alpha_{n}=\infty$; 
(ii) $\lim _{n \rightarrow \infty} \frac{1}{\alpha_{n}}\left|\frac{1}{\beta_{n}}-\frac{1}{\beta_{n-1}}\right|=0, \lim _{n \rightarrow \infty} \frac{1}{\beta_{n}}\left|1-\frac{\alpha_{n-1}}{\alpha_{n}}\right|=0$;

(iii) $\lim _{n \rightarrow \infty} \beta_{n}=0, \lim _{n \rightarrow \infty} \frac{\alpha_{n}}{\beta_{n}}=0$ and $\lim _{n \rightarrow \infty} \frac{\beta_{n}^{2}}{\alpha_{n}}=0$;

(iv) $\operatorname{Fix}(T) \cap$ int $C \neq \emptyset$;

(v) there exists a constant $k>0$ such that $\|x-T x\| \geq k \operatorname{Dist}(x$, Fix $(T))$ for each $x \in C$, where $\operatorname{Dist}(x, \operatorname{Fix}(T))=\inf _{y \in \operatorname{Fix}(T)}\|x-y\|$.

Then $\left\{x_{n}\right\}$ converges strongly to $\tilde{x}=P_{\Lambda} V \tilde{x}$ which solves the HVIP

$$
\langle(I-S) \tilde{x}, p-\tilde{x}\rangle \leq 0, \quad \forall p \in \operatorname{Fix}(T) .
$$

Furthermore, let $\varphi: C \rightarrow \mathbf{R}$ be a real-valued function, $A: H \rightarrow H$ be a nonlinear mapping and $\Theta: C \times C \rightarrow \mathbf{R}$ be a bifunction. In 2008, Peng and Yao [9] introduced the generalized mixed equilibrium problem (GMEP) of finding $x \in C$ such that

$$
\Theta(x, y)+\varphi(y)-\varphi(x)+\langle A x, y-x\rangle \geq 0, \quad \forall y \in C .
$$

We denote the set of solutions of $\operatorname{GMEP}(1.9)$ by $\operatorname{GMEP}(\Theta, \varphi, A)$. The $\operatorname{GMEP}(1.9)$ is very general in the sense that it includes, as special cases, optimization problems, variational inequalities, minimax problems, Nash equilibrium problems in noncooperative games and others. The GMEP is further considered and studied; see e.g., [7, 10, 15, 17, 19, 22-24]. In particular, if $\varphi=0$, then GMEP (1.9) reduces to the generalized equilibrium problem (GEP) which is to find $x \in C$ such that

$$
\Theta(x, y)+\langle A x, y-x\rangle \geq 0, \quad \forall y \in C .
$$

It was introduced and studied by Takahashi and Takahashi [25]. The set of solutions of GEP is denoted by $\operatorname{GEP}(\Theta, A)$.

If $A=0$, then GMEP (1.9) reduces to the mixed equilibrium problem (MEP) which is to find $x \in C$ such that

$$
\Theta(x, y)+\varphi(y)-\varphi(x) \geq 0, \quad \forall y \in C .
$$

It was considered and studied in [26]. The set of solutions of MEP is denoted by $\operatorname{MEP}(\Theta, \varphi)$.

If $\varphi=0, A=0$, then GMEP (1.9) reduces to the equilibrium problem (EP) which is to find $x \in C$ such that

$$
\Theta(x, y) \geq 0, \quad \forall y \in C .
$$

It was considered and studied in $[27,28]$. The set of solutions of EP is denoted by $\operatorname{EP}(\Theta)$. It is worth to mention that the EP is a unified model of several problems, namely, variational inequality problems, optimization problems, saddle point problems, complementarity problems, fixed point problems, Nash equilibrium problems, etc.

It was assumed in [9] that $\Theta: C \times C \rightarrow \mathbf{R}$ is a bifunction satisfying conditions (A1)-(A4) and $\varphi: C \rightarrow \mathbf{R}$ is a lower semicontinuous and convex function with restriction (B1) or (B2), where

(A1) $\Theta(x, x)=0$ for all $x \in C$;

(A2) $\Theta$ is monotone, i.e., $\Theta(x, y)+\Theta(y, x) \leq 0$ for any $x, y \in C$; 
(A3) $\Theta$ is upper-hemicontinuous, i.e., for each $x, y, z \in C$,

$$
\limsup _{t \rightarrow 0^{+}} \Theta(t z+(1-t) x, y) \leq \Theta(x, y)
$$

(A4) $\Theta(x, \cdot)$ is convex and lower semicontinuous for each $x \in C$;

(B1) for each $x \in H$ and $r>0$, there exists a bounded subset $D_{x} \subset C$ and $y_{x} \in C$ such that, for any $z \in C \backslash D_{x}$,

$$
\Theta\left(z, y_{x}\right)+\varphi\left(y_{x}\right)-\varphi(z)+\frac{1}{r}\left\langle y_{x}-z, z-x\right\rangle<0
$$

(B2) $C$ is a bounded set.

Given a positive number $r>0$. Let $T_{r}^{(\Theta, \varphi)}: H \rightarrow C$ be the solution set of the auxiliary mixed equilibrium problem, that is, for each $x \in H$,

$$
T_{r}^{(\Theta, \varphi)}(x):=\left\{y \in C: \Theta(y, z)+\varphi(z)-\varphi(y)+\frac{1}{r}\langle y-x, z-y\rangle \geq 0, \forall z \in C\right\} .
$$

In addition, let $B$ be a single-valued mapping of $C$ into $H$ and $R$ be a multivalued mapping with $D(R)=C$. Consider the following variational inclusion: find a point $x \in C$ such that

$$
0 \in B x+R x
$$

We denote by $\mathrm{I}(B, R)$ the solution set of the variational inclusion (1.10). In particular, if $B=R=0$, then $\mathrm{I}(B, R)=C$. If $B=0$, then problem (1.10) becomes the inclusion problem introduced by Rockafellar [29]. It is known that problem (1.10) provides a convenient framework for the unified study of optimal solutions in many optimization related areas including mathematical programming, complementarity problems, variational inequalities, optimal control, mathematical economics, equilibria, and game theory, etc. Let a set-valued mapping $R: D(R) \subset H \rightarrow 2^{H}$ be maximal monotone. We define the resolvent operator $J_{R, \lambda}: H \rightarrow \overline{D(R)}$ associated with $R$ and $\lambda$ as follows:

$$
J_{R, \lambda}=(I+\lambda R)^{-1}, \quad \forall x \in H,
$$

where $\lambda$ is a positive number.

In 1998, Huang [30] studied problem (1.10) in the case where $R$ is maximal monotone and $B$ is strongly monotone and Lipschitz-continuous with $D(R)=C=H$. Subsequently, Zeng et al. [31] further studied problem (1.10) in the case which is more general than Huang's one [30]. Moreover, the authors [31] obtained the same strong convergence conclusion as in Huang's result [30]. In addition, the authors also gave a geometric convergence rate estimate for approximate solutions. Also, various types of iterative algorithms for solving variational inclusions have been further studied and developed; for more details, refer to [12, 24, 32-34] and the references therein.

Motivated and inspired by the above facts, we introduce and analyze a multistep hybrid extragradient algorithm by combining Korpelevich's extragradient method, the viscosity approximation method, thehybrid steepest-descent method, Mann's iteration method, and the gradient-projection method (GPM) with regularization in the setting of infinitedimensional Hilbert spaces. It is proven that under appropriate assumptions the proposed 
algorithm converges strongly to a solution of the CMP (1.2) with constraints of several problems: finitely many GMEPs, finitely many variational inclusions, and the fixed point problem of a strictly pseudocontractive mapping. In the meantime, we also prove the strong convergence of the proposed algorithm to the unique solution of a hierarchical variational inequality problem (over the fixed point set of a strictly pseudocontractive mapping) with constraints of finitely many GMEPs, finitely many variational inclusions, and the CMP (1.2). Our results represent the supplementation, improvement, extension, and development of the corresponding results in $\mathrm{Xu}$ [20, Theorems 4.1 and 5.2] and Yao et al. [21, Theorems 3.1 and 3.2].

\section{Preliminaries}

Throughout this paper, we assume that $H$ is a real Hilbert space whose inner product and norm are denoted by $\langle\cdot, \cdot\rangle$ and $\|\cdot\|$, respectively. Let $C$ be a nonempty closed convex subset of $H$. We write $x_{n} \rightarrow x$ to indicate that the sequence $\left\{x_{n}\right\}$ converges weakly to $x$ and $x_{n} \rightarrow x$ to indicate that the sequence $\left\{x_{n}\right\}$ converges strongly to $x$. Moreover, we use $\omega_{w}\left(x_{n}\right)$ to denote the weak $\omega$-limit set of the sequence $\left\{x_{n}\right\}$, i.e.,

$$
\omega_{w}\left(x_{n}\right):=\left\{x \in H: x_{n_{i}} \rightarrow x \text { for some subsequence }\left\{x_{n_{i}}\right\} \text { of }\left\{x_{n}\right\}\right\} .
$$

The metric (or nearest point) projection from $H$ onto $C$ is the mapping $P_{C}: H \rightarrow C$ which assigns to each point $x \in H$ the unique point $P_{C} x \in C$ satisfying the property

$$
\left\|x-P_{C} x\right\|=\inf _{y \in C}\|x-y\|=: d(x, C)
$$

Definition 2.1 Let $T$ be a nonlinear operator with the domain $D(T) \subset H$ and the range $R(T) \subset H$. Then $T$ is said to be

(i) monotone if

$$
\langle T x-T y, x-y\rangle \geq 0, \quad \forall x, y \in D(T)
$$

(ii) $\beta$-strongly monotone if there exists a constant $\beta>0$ such that

$$
\langle T x-T y, x-y\rangle \geq \eta\|x-y\|^{2}, \quad \forall x, y \in D(T)
$$

(iii) $v$-inverse-strongly monotone if there exists a constant $v>0$ such that

$$
\langle T x-T y, x-y\rangle \geq v\|T x-T y\|^{2}, \quad \forall x, y \in D(T) .
$$

It is easy to see that the projection $P_{C}$ is 1-inverse-strongly monotone. Inverse-strongly monotone (also referred to as co-coercive) operators have been applied widely in solving practical problems in various fields, for instance, in traffic assignment problems; see e.g., [35]. It is obvious that if $T$ is $v$-inverse-strongly monotone, then $T$ is monotone and $\frac{1}{v}$ Lipschitz-continuous. Moreover, we also have, for all $u, v \in D(T)$ and $\lambda>0$,

$$
\begin{aligned}
& \|(I-\lambda T) u-(I-\lambda T) v\|^{2} \\
& \quad=\|(u-v)-\lambda(T u-T v)\|^{2}
\end{aligned}
$$




$$
\begin{aligned}
& =\|u-v\|^{2}-2 \lambda\langle T u-T v, u-v\rangle+\lambda^{2}\|T u-T v\|^{2} \\
& \leq\|u-v\|^{2}+\lambda(\lambda-2 v)\|T u-T v\|^{2} .
\end{aligned}
$$

So, if $\lambda \leq 2 v$, then $I-\lambda T$ is a nonexpansive mapping.

Some important properties of projections are gathered in the following proposition.

Proposition 2.1 For given $x \in H$ and $z \in C$ :

(i) $z=P_{C} x \Leftrightarrow\langle x-z, y-z\rangle \leq 0, \forall y \in C$;

(ii) $z=P_{C} x \Leftrightarrow\|x-z\|^{2} \leq\|x-y\|^{2}-\|y-z\|^{2}, \forall y \in C$;

(iii) $\left\langle P_{C} x-P_{C} y, x-y\right\rangle \geq\left\|P_{C} x-P_{C} y\right\|^{2}, \forall y \in H$.

Consequently, $P_{C}$ is nonexpansive and monotone.

Definition 2.2 A mapping $T: H \rightarrow H$ is said to be

(a) nonexpansive if

$$
\|T x-T y\| \leq\|x-y\|, \quad \forall x, y \in H
$$

(b) firmly nonexpansive if $2 T-I$ is nonexpansive, or equivalently, if $T$ is 1 -inverse-strongly monotone (1-ism),

$$
\langle x-y, T x-T y\rangle \geq\|T x-T y\|^{2}, \quad \forall x, y \in H
$$

alternatively, $T$ is firmly nonexpansive if and only if $T$ can be expressed as

$$
T=\frac{1}{2}(I+S)
$$

where $S: H \rightarrow H$ is nonexpansive; projections are firmly nonexpansive.

It can easily be seen that if $T$ is nonexpansive, then $I-T$ is monotone.

Next we list some elementary conclusions for the MEP.

Proposition 2.2 (see [26]) Assume that $\Theta: C \times C \rightarrow \mathbf{R}$ satisfies (A1)-(A4) and let $\varphi: C \rightarrow$ $\mathbf{R}$ be a proper lower semicontinuous and convex function. Assume that either (B1) or (B2) holds. For $r>0$ and $x \in H$, define a mapping $T_{r}^{(\Theta, \varphi)}: H \rightarrow C$ as follows:

$$
T_{r}^{(\Theta, \varphi)}(x)=\left\{z \in C: \Theta(z, y)+\varphi(y)-\varphi(z)+\frac{1}{r}\langle y-z, z-x\rangle \geq 0, \forall y \in C\right\}
$$

for all $x \in H$. Then the following hold:

(i) for each $x \in H, T_{r}^{(\Theta, \varphi)}(x)$ is nonempty and single-valued;

(ii) $T_{r}^{(\Theta, \varphi)}$ is firmly nonexpansive, that is, for any $x, y \in H$,

$$
\left\|T_{r}^{(\Theta, \varphi)} x-T_{r}^{(\Theta, \varphi)} y\right\|^{2} \leq\left\langle T_{r}^{(\Theta, \varphi)} x-T_{r}^{(\Theta, \varphi)} y, x-y\right\rangle
$$

(iii) $\operatorname{Fix}\left(T_{r}^{(\Theta, \varphi)}\right)=\operatorname{MEP}(\Theta, \varphi)$;

(iv) $\operatorname{MEP}(\Theta, \varphi)$ is closed and convex;

(v) $\left\|T_{s}^{(\Theta, \varphi)} x-T_{t}^{(\Theta, \varphi)} x\right\|^{2} \leq \frac{s-t}{s}\left\langle T_{s}^{(\Theta, \varphi)} x-T_{t}^{(\Theta, \varphi)} x, T_{s}^{(\Theta, \varphi)} x-x\right\rangle$ for all $s, t>0$ and $x \in H$. 
Definition 2.3 A mapping $T: H \rightarrow H$ is said to be an averaged mapping if it can be written as the average of the identity $I$ and a nonexpansive mapping, that is,

$$
T \equiv(1-\alpha) I+\alpha S,
$$

where $\alpha \in(0,1)$ and $S: H \rightarrow H$ is nonexpansive. More precisely, when the last equality holds, we say that $T$ is $\alpha$-averaged. Thus firmly nonexpansive mappings (in particular, projections) are $\frac{1}{2}$-averaged mappings.

Proposition 2.3 (see [36]) Let $T: H \rightarrow H$ be a given mapping.

(i) $T$ is nonexpansive if and only if the complement $I-T$ is $\frac{1}{2}-$ ism.

(ii) If $T$ is $v$-ism, then for $\gamma>0, \gamma T$ is $\frac{v}{\gamma}$-ism.

(iii) $T$ is averaged if and only if the complement $I-T$ is $v$-ism for some $v>1 / 2$. Indeed, for $\alpha \in(0,1), T$ is $\alpha$-averaged if and only if $I-T$ is $\frac{1}{2 \alpha}$-ism.

Proposition 2.4 (see $[36,37]$ ) Let $S, T, V: H \rightarrow H$ be given operators.

(i) If $T=(1-\alpha) S+\alpha V$ for some $\alpha \in(0,1)$ and if $S$ is averaged and $V$ is nonexpansive, then $T$ is averaged.

(ii) $T$ is firmly nonexpansive if and only if the complement $I-T$ is firmly nonexpansive.

(iii) If $T=(1-\alpha) S+\alpha V$ for some $\alpha \in(0,1)$ and if $S$ is firmly nonexpansive and $V$ is nonexpansive, then $T$ is averaged.

(iv) The composite of finitely many averaged mappings is averaged. That is, if each of the mappings $\left\{T_{i}\right\}_{i=1}^{N}$ is averaged, then so is the composite $T_{1} \cdots T_{N}$. In particular, if $T_{1}$ is $\alpha_{1}$-averaged and $T_{2}$ is $\alpha_{2}$-averaged, where $\alpha_{1}, \alpha_{2} \in(0,1)$, then the composite $T_{1} T_{2}$ is $\alpha$-averaged, where $\alpha=\alpha_{1}+\alpha_{2}-\alpha_{1} \alpha_{2}$.

(v) If the mappings $\left\{T_{i}\right\}_{i=1}^{N}$ are averaged and have a common fixed point, then

$$
\bigcap_{i=1}^{N} \operatorname{Fix}\left(T_{i}\right)=\operatorname{Fix}\left(T_{1} \cdots T_{N}\right)
$$

The notation $\operatorname{Fix}(T)$ denotes the set of all fixed points of the mapping $T$, that is, $\operatorname{Fix}(T)=\{x \in H: T x=x\}$.

We need some facts and tools in a real Hilbert space $H$ which are listed as lemmas below.

Lemma 2.1 Let $X$ be a real inner product space. Then we have the following inequality:

$$
\|x+y\|^{2} \leq\|x\|^{2}+2\langle y, x+y\rangle, \quad \forall x, y \in X .
$$

Lemma 2.2 Let $H$ be a real Hilbert space. Then the following hold:

(a) $\|x-y\|^{2}=\|x\|^{2}-\|y\|^{2}-2\langle x-y, y\rangle$ for all $x, y \in H$;

(b) $\|\lambda x+\mu y\|^{2}=\lambda\|x\|^{2}+\mu\|y\|^{2}-\lambda \mu\|x-y\|^{2}$ for all $x, y \in H$ and $\lambda, \mu \in[0,1]$ with $\lambda+\mu=1$

(c) if $\left\{x_{n}\right\}$ is a sequence in $H$ such that $x_{n} \rightarrow x$, it follows that

$$
\limsup _{n \rightarrow \infty}\left\|x_{n}-y\right\|^{2}=\limsup _{n \rightarrow \infty}\left\|x_{n}-x\right\|^{2}+\|x-y\|^{2}, \quad \forall y \in H .
$$


It is clear that, in a real Hilbert space $H, T: C \rightarrow C$ is $\xi$-strictly pseudocontractive if and only if the following inequality holds:

$$
\langle T x-T y, x-y\rangle \leq\|x-y\|^{2}-\frac{1-\xi}{2}\|(I-T) x-(I-T) y\|^{2}, \quad \forall x, y \in C .
$$

This immediately implies that if $T$ is a $\xi$-strictly pseudocontractive mapping, then $I-T$ is $\frac{1-\xi}{2}$-inverse strongly monotone; for further details, we refer to [38] and the references therein. It is well known that the class of strict pseudocontractions strictly includes the class of nonexpansive mappings and that the class of pseudocontractions strictly includes the class of strict pseudocontractions.

Lemma 2.3 (see [38, Proposition 2.1]) Let C be a nonempty closed convex subset of a real Hilbert space $H$ and $T: C \rightarrow C$ be a mapping.

(i) If $T$ is a $\xi$-strictly pseudocontractive mapping, then $T$ satisfies the Lipschitzian condition

$$
\|T x-T y\| \leq \frac{1+\xi}{1-\xi}\|x-y\|, \quad \forall x, y \in C .
$$

(ii) If $T$ is a $\xi$-strictly pseudocontractive mapping, then the mapping $I-T$ is semiclosed at 0 , that is, if $\left\{x_{n}\right\}$ is a sequence in $C$ such that $x_{n} \rightarrow \tilde{x}$ and $(I-T) x_{n} \rightarrow 0$, then $(I-T) \tilde{x}=0$.

(iii) If $T$ is $\xi$-(quasi-)strict pseudocontraction, then the fixed point set $\operatorname{Fix}(T)$ of $T$ is closed and convex so that the projection $P_{\mathrm{Fix}(T)}$ is well defined.

Lemma 2.4 (see [11]) Let $C$ be a nonempty closed convex subset of a real Hilbert space $H$. Let $T: C \rightarrow C$ be a $\xi$-strictly pseudocontractive mapping. Let $\gamma$ and $\delta$ be two nonnegative real numbers such that $(\gamma+\delta) \xi \leq \gamma$. Then

$$
\|\gamma(x-y)+\delta(T x-T y)\| \leq(\gamma+\delta)\|x-y\|, \quad \forall x, y \in C .
$$

Lemma 2.5 (see [39, Demiclosedness principle]) Let C be a nonempty closed convex subset of a real Hilbert space $H$. Let $S$ be a nonexpansive self-mapping on $C$ with $\operatorname{Fix}(S) \neq \emptyset$. Then $I-S$ is demiclosed. That is, whenever $\left\{x_{n}\right\}$ is a sequence in $C$ weakly converging to some $x \in C$ and the sequence $\left\{(I-S) x_{n}\right\}$ strongly converges to some $y$, it follows that $(I-S) x=y$. Here I is the identity operator of $H$.

Lemma 2.6 Let $A: C \rightarrow H$ be a monotone mapping. In the context of the variational inequality problem the characterization of the projection (see Proposition 2.1(i)) implies

$$
u \in \operatorname{VI}(C, A) \quad \Leftrightarrow \quad u=P_{C}(u-\lambda A u), \quad \lambda>0 .
$$

Let $C$ be a nonempty closed convex subset of a real Hilbert space $H$. We introduce some notations. Let $\lambda$ be a number in $(0,1]$ and let $\mu>0$. Associated with a nonexpansive mapping $T: C \rightarrow H$, we define the mapping $T^{\lambda}: C \rightarrow H$ by

$$
T^{\lambda} x:=T x-\lambda \mu F(T x), \quad \forall x \in C,
$$


where $F: H \rightarrow H$ is an operator such that, for some positive constants $\kappa, \eta>0, F$ is $\kappa$-Lipschitzian and $\eta$-strongly monotone on $H$; that is, $F$ satisfies the conditions:

$$
\|F x-F y\| \leq \kappa\|x-y\| \quad \text { and } \quad\langle F x-F y, x-y\rangle \geq \eta\|x-y\|^{2}
$$

for all $x, y \in H$.

Lemma 2.7 (see [40, Lemma 3.1]) $T^{\lambda}$ is a contraction provided $0<\mu<\frac{2 \eta}{\kappa^{2}}$; that is,

$$
\left\|T^{\lambda} x-T^{\lambda} y\right\| \leq(1-\lambda \tau)\|x-y\|, \quad \forall x, y \in C,
$$

where $\tau=1-\sqrt{1-\mu\left(2 \eta-\mu \kappa^{2}\right)} \in(0,1]$.

Remark 2.1 (i) Since $F$ is $\kappa$-Lipschitzian and $\eta$-strongly monotone on $H$, we get $0<\eta \leq \kappa$. Hence, whenever $0<\mu<\frac{2 \eta}{\kappa^{2}}$, we have $\tau=1-\sqrt{1-\mu\left(2 \eta-\mu \kappa^{2}\right)} \in(0,1]$.

(ii) In Lemma 2.7, put $F=\frac{1}{2} I$ and $\mu=2$. Then we know that $\kappa=\eta=\frac{1}{2}, 0<\mu=2<\frac{2 \eta}{\kappa^{2}}=4$, and $\tau=1$.

Lemma 2.8 (see [41]) Let $\left\{a_{n}\right\}$ be a sequence of nonnegative real numbers satisfying the property:

$$
a_{n+1} \leq\left(1-s_{n}\right) a_{n}+s_{n} b_{n}+t_{n}, \quad \forall n \geq 0,
$$

where $\left\{s_{n}\right\} \subset(0,1]$ and $\left\{b_{n}\right\}$ are such that:

(i) $\sum_{n=0}^{\infty} s_{n}=\infty$;

(ii) either $\limsup _{n \rightarrow \infty} b_{n} \leq 0$ or $\sum_{n=0}^{\infty}\left|s_{n} b_{n}\right|<\infty$;

(iii) $\sum_{n=0}^{\infty} t_{n}<\infty$ where $t_{n} \geq 0$, for all $n \geq 0$.

Then $\lim _{n \rightarrow \infty} a_{n}=0$.

Recall that a set-valued mapping $T: D(T) \subset H \rightarrow 2^{H}$ is called monotone if for all $x, y \in$ $D(T), f \in T x$ and $g \in T y$ imply

$$
\langle f-g, x-y\rangle \geq 0
$$

A set-valued mapping $T$ is called maximal monotone if $T$ is monotone and $(I+\lambda T) D(T)=$ $H$ for each $\lambda>0$, where $I$ is the identity mapping of $H$. We denote by $G(T)$ the graph of $T$. It is well known that a monotone mapping $T$ is maximal if and only if, for $(x, f) \in H \times H$, $\langle f-g, x-y\rangle \geq 0$ for every $(y, g) \in G(T)$ implies $f \in T x$. Next we provide an example to illustrate the concept of a maximal monotone mapping.

Let $A: C \rightarrow H$ be a monotone, $k$-Lipschitz-continuous mapping and let $N_{C} v$ be the normal cone to $C$ at $v \in C$, i.e.,

$$
N_{C} v=\{u \in H:\langle v-p, u\rangle \geq 0, \forall p \in C\} .
$$

Define

$$
\widetilde{T} v= \begin{cases}A v+N_{C} v, & \text { if } v \in C, \\ \emptyset, & \text { if } v \notin C .\end{cases}
$$


Then $\widetilde{T}$ is maximal monotone (see [29]) such that

$$
0 \in \widetilde{T} v \quad \Leftrightarrow \quad v \in \operatorname{VI}(C, A) .
$$

Let $R: D(R) \subset H \rightarrow 2^{H}$ be a maximal monotone mapping. Let $\lambda, \mu>0$ be two positive numbers.

Lemma 2.9 (see [42]) We have the resolvent identity

$$
J_{R, \lambda} x=J_{R, \mu}\left(\frac{\mu}{\lambda} x+\left(1-\frac{\mu}{\lambda}\right) J_{R, \lambda} x\right), \quad \forall x \in H
$$

Remark 2.2 For $\lambda, \mu>0$, we have the following relation:

$$
\left\|J_{R, \lambda} x-J_{R, \mu} y\right\| \leq\|x-y\|+|\lambda-\mu|\left(\frac{1}{\lambda}\left\|J_{R, \lambda} x-y\right\|+\frac{1}{\mu}\left\|x-J_{R, \mu} y\right\|\right), \quad \forall x, y \in H .
$$

In terms of Huang [30] (see also [31]), we have the following property for the resolvent operator $J_{R, \lambda}: H \rightarrow \overline{D(R)}$.

Lemma 2.10 $J_{R, \lambda}$ is single-valued and firmly nonexpansive, i.e.,

$$
\left\langle J_{R, \lambda} x-J_{R, \lambda} y, x-y\right\rangle \geq\left\|J_{R, \lambda} x-J_{R, \lambda} y\right\|^{2}, \quad \forall x, y \in H .
$$

Consequently, $J_{R, \lambda}$ is nonexpansive and monotone.

Lemma 2.11 (see [12]) Let $R$ be a maximal monotone mapping with $D(R)=C$. Then for any given $\lambda>0, u \in C$ is a solution of problem (1.5) if and only if $u \in C$ satisfies

$$
u=J_{R, \lambda}(u-\lambda B u) .
$$

Lemma 2.12 (see [31]) Let $R$ be a maximal monotone mapping with $D(R)=C$ and let $B: C \rightarrow H$ be a strongly monotone, continuous, and single-valued mapping. Then for each $z \in H$, the equation $z \in(B+\lambda R) x$ has a unique solution $x_{\lambda}$ for $\lambda>0$.

Lemma 2.13 (see [12]) Let $R$ be a maximal monotone mapping with $D(R)=C$ and $B$ : $C \rightarrow H$ be a monotone, continuous and single-valued mapping. Then $(I+\lambda(R+B)) C=H$ for each $\lambda>0$. In this case, $R+B$ is maximal monotone.

\section{Main results}

In this section, we will introduce and analyze a multistep hybrid extragradient algorithm for finding a solution of the CMP (1.2) with constraints of several problems: finitely many GMEPs and finitely many variational inclusions and the fixed point problem of a strict pseudocontraction in a real Hilbert space. This algorithm is based on Korpelevich's extragradient method, the viscosity approximation method, the hybrid steepest-descent method [43], Mann's iteration method and the gradient-projection method (GPM) with regularization. Under appropriate assumptions, we prove the strong convergence of the 
proposed algorithm to a solution of the CMP (1.2), which is also the unique solution of a hierarchical variational inequality problem (HVIP).

Let $C$ be a nonempty closed convex subset of a real Hilbert space $H$ and $f: C \rightarrow \mathbf{R}$ be a convex functional with $L$-Lipschitz-continuous gradient $\nabla f$. We denote by $\Gamma$ the solution set of the CMP (1.2). Then the CMP (1.2) is generally ill-posed. Consider the following Tikhonov regularization problem:

$$
\min _{x \in C} f_{\alpha}(x):=f(x)+\frac{1}{2} \alpha\|x\|^{2},
$$

where $\alpha>0$ is the regularization parameter. Hence, we have

$$
\nabla f_{\alpha}=\nabla f+\alpha I
$$

We are now in a position to state and prove the main result in this paper.

Theorem 3.1 Let $C$ be a nonempty closed convex subset of a real Hilbert space H. Let $M, N$ be two positive integers. Let $f: C \rightarrow \mathbf{R}$ be a convex functional with L-Lipschitz-continuous gradient $\nabla f$. Let $\Theta_{k}$ be a bifunction from $C \times C$ to $\mathbf{R}$ satisfying (A1)-(A4) and $\varphi_{k}: C \rightarrow$ $\mathbf{R} \cup\{+\infty\}$ be a proper lower semicontinuous and convex function with restriction (B1) or (B2), where $k \in\{1,2, \ldots, M\}$. Let $R_{i}: C \rightarrow 2^{H}$ be a maximal monotone mapping and let $A_{k}$ : $H \rightarrow H$ and $B_{i}: C \rightarrow H$ be $\mu_{k}$-inverse-strongly monotone and $\eta_{i}$-inverse-strongly monotone, respectively, where $k \in\{1,2, \ldots, M\}, i \in\{1,2, \ldots, N\}$. Let $T: C \rightarrow C$ be a $\xi$-strictly pseudocontractive mapping, $S: H \rightarrow H$ be a nonexpansive mapping and $V: H \rightarrow H$ be a $\rho$-contraction with coefficient $\rho \in[0,1)$. Let $F: H \rightarrow H$ be $\kappa$-Lipschitzian and $\eta$-strongly monotone with positive constants $\kappa, \eta>0$ such that $0 \leq \gamma<\tau$ and $0<\mu<\frac{2 \eta}{\kappa^{2}}$ where $\tau=$ $1-\sqrt{1-\mu\left(2 \eta-\mu \kappa^{2}\right)}$. Assume that $\Omega:=\bigcap_{k=1}^{M} \operatorname{GMEP}\left(\Theta_{k}, \varphi_{k}, A_{k}\right) \cap \bigcap_{i=1}^{N} \mathrm{I}\left(B_{i}, R_{i}\right) \cap \operatorname{Fix}(T) \cap$ $\Gamma \neq \emptyset$. Let $\left\{\lambda_{n}\right\} \subset[a, b] \subset\left(0, \frac{2}{L}\right),\left\{\alpha_{n}\right\} \subset(0, \infty)$ with $\sum_{n=0}^{\infty} \alpha_{n}<\infty,\left\{\epsilon_{n}\right\},\left\{\delta_{n}\right\},\left\{\beta_{n}\right\},\left\{\gamma_{n}\right\}$, $\left\{\sigma_{n}\right\} \subset(0,1)$ with $\beta_{n}+\gamma_{n}+\sigma_{n}=1$, and $\left\{\lambda_{i, n}\right\} \subset\left[a_{i}, b_{i}\right] \subset\left(0,2 \eta_{i}\right),\left\{r_{k, n}\right\} \subset\left[c_{k}, d_{k}\right] \subset\left(0,2 \mu_{k}\right)$ where $i \in\{1,2, \ldots, N\}$ and $k \in\{1,2, \ldots, M\}$. For arbitrarily given $x_{0} \in H$, let $\left\{x_{n}\right\}$ be a sequence generated by

$$
\left\{\begin{array}{l}
u_{n}=T_{r_{M, n}}^{\left(\Theta_{M}, \varphi_{M}\right)}\left(I-r_{M, n} A_{M}\right) T_{r_{M-1, n}}^{\left(\Theta_{M-1}, \varphi_{M-1}\right)}\left(I-r_{M-1, n} A_{M-1}\right) \cdots T_{r_{1, n}}^{\left(\Theta_{1}, \varphi_{1}\right)}\left(I-r_{1, n} A_{1}\right) x_{n}, \\
v_{n}=J_{R_{N}, \lambda_{N, n}}\left(I-\lambda_{N, n} B_{N}\right) J_{R_{N-1}, \lambda_{N-1, n}}\left(I-\lambda_{N-1, n} B_{N-1}\right) \cdots J_{R_{1}, \lambda_{1, n}}\left(I-\lambda_{1, n} B_{1}\right) u_{n}, \\
y_{n}=\beta_{n} x_{n}+\gamma_{n} P_{C}\left(I-\lambda_{n} \nabla f_{\alpha_{n}}\right) v_{n}+\sigma_{n} T P_{C}\left(I-\lambda_{n} \nabla f_{\alpha_{n}}\right) v_{n} \\
x_{n+1}=\epsilon_{n} \gamma\left(\delta_{n} V x_{n}+\left(1-\delta_{n}\right) S x_{n}\right)+\left(I-\epsilon_{n} \mu F\right) y_{n}, \quad \forall n \geq 0
\end{array}\right.
$$

where $\nabla f_{\alpha_{n}}=\alpha_{n} I+\nabla f$ for all $n \geq 0$. Suppose that

(C1) $\lim _{n \rightarrow \infty} \epsilon_{n}=0, \sum_{n=0}^{\infty} \epsilon_{n}=\infty$ and $\lim _{n \rightarrow \infty} \frac{1}{\epsilon_{n}}\left|1-\frac{\delta_{n-1}}{\delta_{n}}\right|=0$;

(C2) $\lim \sup _{n \rightarrow \infty} \frac{\delta_{n}}{\epsilon_{n}}<\infty, \lim _{n \rightarrow \infty} \frac{1}{\epsilon_{n}}\left|\frac{1}{\delta_{n}}-\frac{1}{\delta_{n-1}}\right|=0$ and $\lim _{n \rightarrow \infty} \frac{1}{\delta_{n}}\left|1-\frac{\epsilon_{n-1}}{\epsilon_{n}}\right|=0$;

(C3) $\lim _{n \rightarrow \infty} \frac{\left|\beta_{n}-\beta_{n-1}\right|}{\epsilon_{n} \delta_{n}}=0$ and $\lim _{n \rightarrow \infty} \frac{\left|\gamma_{n}-\gamma_{n-1}\right|}{\epsilon_{n} \delta_{n}}=0$;

(C4) $\lim _{n \rightarrow \infty} \frac{\left|\lambda_{i, n}-\lambda_{i, n-1}\right|}{\epsilon_{n} \delta_{n}}=0$ and $\lim _{n \rightarrow \infty} \frac{\left|r_{k, n}-r_{k, n-1}\right|}{\epsilon_{n} \delta_{n}}=0$ for $i=1,2, \ldots, N$ and $k=1,2, \ldots, M$

(C5) $\lim _{n \rightarrow \infty} \frac{\left|\lambda_{n}-\lambda_{n-1}\right|}{\epsilon_{n} \delta_{n}}=0, \lim _{n \rightarrow \infty} \frac{\left|\lambda_{n} \alpha_{n}-\lambda_{n-1} \alpha_{n-1}\right|}{\epsilon_{n} \delta_{n}}=0$ and $\left(\gamma_{n}+\sigma_{n}\right) \xi \leq \gamma_{n}$ for all $n \geq 0$;

(C6) $\left\{\beta_{n}\right\} \subset[c, d] \subset(0,1), \lim _{n \rightarrow \infty} \frac{\alpha_{n}}{\delta_{n}}=0$ and $\liminf _{n \rightarrow \infty} \sigma_{n}>0$.

Then we have:

(i) $\lim _{n \rightarrow \infty} \frac{\left\|x_{n+1}-x_{n}\right\|}{\delta_{n}}=0$; 
(ii) $\omega_{w}\left(x_{n}\right) \subset \Omega$;

(iii) $\left\{x_{n}\right\}$ converges strongly to a minimizer $x^{*}$ of the CMP (1.2), which is a unique solution $x^{*}$ in $\Omega$ to the HVIP

$$
\left\langle(\mu F-\gamma S) x^{*}, p-x^{*}\right\rangle \geq 0, \quad \forall p \in \Omega
$$

Proof First of all, observe that

$$
\begin{aligned}
\mu \eta \geq \tau & \Leftrightarrow \mu \eta \geq 1-\sqrt{1-\mu\left(2 \eta-\mu \kappa^{2}\right)} \\
& \Leftrightarrow \quad \sqrt{1-\mu\left(2 \eta-\mu \kappa^{2}\right)} \geq 1-\mu \eta \\
& \Leftrightarrow 1-2 \mu \eta+\mu^{2} \kappa^{2} \geq 1-2 \mu \eta+\mu^{2} \eta^{2} \\
& \Leftrightarrow \quad \kappa^{2} \geq \eta^{2} \\
& \Leftrightarrow \kappa \geq \eta
\end{aligned}
$$

and

$$
\begin{aligned}
& \langle(\mu F-\gamma S) x-(\mu F-\gamma S) y, x-y\rangle \\
& \quad=\mu\langle F x-F y, x-y\rangle-\gamma\langle S x-S y, x-y\rangle \\
& \quad \geq \mu \eta\|x-y\|^{2}-\gamma\|x-y\|^{2} \\
& \quad=(\mu \eta-\gamma)\|x-y\|^{2}, \quad \forall x, y \in H .
\end{aligned}
$$

Since $0 \leq \gamma<\tau$ and $\kappa \geq \eta$, we know that $\mu \eta \geq \tau>\gamma$ and hence the mapping $\mu F-\gamma S$ is $(\mu \eta-\gamma)$-strongly monotone. Moreover, it is clear that the mapping $\mu F-\gamma S$ is $(\mu \kappa+\gamma)$ Lipschitzian. Thus, there exists a unique solution $x^{*}$ in $\Omega$ to the VIP

$$
\left\langle(\mu F-\gamma S) x^{*}, p-x^{*}\right\rangle \geq 0, \quad \forall p \in \Omega \text {. }
$$

That is, $\left\{x^{*}\right\}=\operatorname{VI}(\Omega, \mu F-\gamma S)$. Now, we put

$$
\Delta_{n}^{k}=T_{r_{k, n}}^{\left(\Theta_{k}, \varphi_{k}\right)}\left(I-r_{k, n} A_{k}\right) T_{r_{k-1, n}}^{\left(\Theta_{k-1}, \varphi_{k-1}\right)}\left(I-r_{k-1, n} A_{k-1}\right) \cdots T_{r_{1, n}}^{\left(\Theta_{1}, \varphi_{1}\right)}\left(I-r_{1, n} A_{1}\right) x_{n}
$$

for all $k \in\{1,2, \ldots, M\}$ and $n \geq 1$,

$$
\Lambda_{n}^{i}=J_{R_{i}, \lambda_{i, n}}\left(I-\lambda_{i, n} B_{i}\right) J_{R_{i-1}, \lambda_{i-1, n}}\left(I-\lambda_{i-1, n} B_{i-1}\right) \cdots J_{R_{1}, \lambda_{1, n}}\left(I-\lambda_{1, n} B_{1}\right)
$$

for all $i \in\{1,2, \ldots, N\}, \Delta_{n}^{0}=I$ and $\Lambda_{n}^{0}=I$, where $I$ is the identity mapping on $H$. Then we have $u_{n}=\Delta_{n}^{M} x_{n}$ and $v_{n}=\Lambda_{n}^{N} u_{n}$.

In addition, we show that $P_{C}\left(I-\lambda \nabla f_{\alpha}\right)$ is $\nu$-averaged for each $\lambda \in\left(0, \frac{2}{\alpha+L}\right)$, where

$$
v=\frac{2+\lambda(\alpha+L)}{4} \in(0,1)
$$

Indeed, the Lipschitz continuity of $\nabla f$ implies that $\nabla f$ is $\frac{1}{L}$-ism (see [20] (also [44])); that is,

$$
\langle\nabla f(x)-\nabla f(y), x-y\rangle \geq \frac{1}{L}\|\nabla f(x)-\nabla f(y)\|^{2}
$$


Observe that

$$
\begin{aligned}
(\alpha & +L)\left\langle\nabla f_{\alpha}(x)-\nabla f_{\alpha}(y), x-y\right\rangle \\
& =(\alpha+L)\left[\alpha\|x-y\|^{2}+\langle\nabla f(x)-\nabla f(y), x-y\rangle\right] \\
& =\alpha^{2}\|x-y\|^{2}+\alpha\langle\nabla f(x)-\nabla f(y), x-y\rangle+\alpha L\|x-y\|^{2}+L\langle\nabla f(x)-\nabla f(y), x-y\rangle \\
& \geq \alpha^{2}\|x-y\|^{2}+2 \alpha\langle\nabla f(x)-\nabla f(y), x-y\rangle+\|\nabla f(x)-\nabla f(y)\|^{2} \\
& =\|\alpha(x-y)+\nabla f(x)-\nabla f(y)\|^{2}=\left\|\nabla f_{\alpha}(x)-\nabla f_{\alpha}(y)\right\|^{2} .
\end{aligned}
$$

Therefore, it follows that $\nabla f_{\alpha}=\nabla f+\alpha I$ is $\frac{1}{\alpha+L}$-ism. Thus, by Proposition 2.3(ii), $\lambda \nabla f_{\alpha}$ is $\frac{1}{\lambda(\alpha+L)}$-ism. From Proposition 2.3(iii), the complement $I-\lambda \nabla f_{\alpha}$ is $\frac{\lambda(\alpha+L)}{2}$-averaged. Consequently, noting that $P_{C}$ is $\frac{1}{2}$-averaged and utilizing Proposition 2.4(iv), we find that, for each $\lambda \in\left(0, \frac{2}{\alpha+L}\right), P_{C}\left(I-\lambda \nabla f_{\alpha}\right)$ is $v$-averaged with

$$
v=\frac{1}{2}+\frac{\lambda(\alpha+L)}{2}-\frac{1}{2} \cdot \frac{\lambda(\alpha+L)}{2}=\frac{2+\lambda(\alpha+L)}{4} \in(0,1) .
$$

This shows that $P_{C}\left(I-\lambda \nabla f_{\alpha}\right)$ is nonexpansive. Taking into account that $\left\{\lambda_{n}\right\} \subset[a, b] \subset$ $\left(0, \frac{2}{L}\right)$ and $\alpha_{n} \rightarrow 0$, we get

$$
\limsup _{n \rightarrow \infty} \frac{2+\lambda_{n}\left(\alpha_{n}+L\right)}{4} \leq \frac{2+b L}{4}<1
$$

Without loss of generality, we may assume that $v_{n}:=\frac{2+\lambda_{n}\left(\alpha_{n}+L\right)}{4}<1$ for each $n \geq 0$. So, $P_{C}\left(I-\lambda_{n} \nabla f_{\alpha_{n}}\right)$ is nonexpansive for each $n \geq 0$. Similarly, since

$$
\limsup _{n \rightarrow \infty} \frac{\lambda_{n}\left(\alpha_{n}+L\right)}{2} \leq \frac{b L}{2}<1,
$$

it is well known that $I-\lambda_{n} \nabla f_{\alpha_{n}}$ is nonexpansive for each $n \geq 0$.

We divide the rest of the proof into several steps.

Step 1. We prove that $\left\{x_{n}\right\}$ is bounded.

Indeed, take a fixed $p \in \Omega$ arbitrarily. Utilizing (2.1) and Proposition 2.2(ii) we have

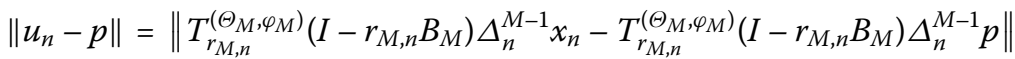

$$
\begin{aligned}
& \leq\left\|\left(I-r_{M, n} B_{M}\right) \Delta_{n}^{M-1} x_{n}-\left(I-r_{M, n} B_{M}\right) \Delta_{n}^{M-1} p\right\| \\
& \leq\left\|\Delta_{n}^{M-1} x_{n}-\Delta_{n}^{M-1} p\right\| \\
& \text {. } \\
& \leq\left\|\Delta_{n}^{0} x_{n}-\Delta_{n}^{0} p\right\| \\
& =\left\|x_{n}-p\right\| \text {. }
\end{aligned}
$$

Utilizing (2.1) and Lemma 2.10 we have

$$
\begin{aligned}
\left\|v_{n}-p\right\| & =\left\|J_{R_{N}, \lambda_{N, n}}\left(I-\lambda_{N, n} A_{N}\right) \Lambda_{n}^{N-1} u_{n}-J_{R_{N}, \lambda_{N, n}}\left(I-\lambda_{N, n} A_{N}\right) \Lambda_{n}^{N-1} p\right\| \\
& \leq\left\|\left(I-\lambda_{N, n} A_{N}\right) \Lambda_{n}^{N-1} u_{n}-\left(I-\lambda_{N, n} A_{N}\right) \Lambda_{n}^{N-1} p\right\|
\end{aligned}
$$




$$
\begin{aligned}
\leq & \left\|\Lambda_{n}^{N-1} u_{n}-\Lambda_{n}^{N-1} p\right\| \\
& \ldots \\
\leq & \left\|\Lambda_{n}^{0} u_{n}-\Lambda_{n}^{0} p\right\| \\
= & \left\|u_{n}-p\right\| .
\end{aligned}
$$

Combining (3.2) and (3.3), we have

$$
\left\|v_{n}-p\right\| \leq\left\|x_{n}-p\right\|
$$

For simplicity, put $t_{n}=P_{C}\left(I-\lambda_{n} \nabla f_{\alpha_{n}}\right) v_{n}$ for each $n \geq 0$. Note that $P_{C}(I-\lambda \nabla f) p=p$ for $\lambda \in\left(0, \frac{2}{L}\right)$. Hence, from (3.4), it follows that

$$
\begin{aligned}
& \left\|t_{n}-p\right\| \\
& \quad=\left\|P_{C}\left(I-\lambda_{n} \nabla f_{\alpha_{n}}\right) v_{n}-P_{C}\left(I-\lambda_{n} \nabla f\right) p\right\| \\
& \quad \leq\left\|P_{C}\left(I-\lambda_{n} \nabla f_{\alpha_{n}}\right) v_{n}-P_{C}\left(I-\lambda_{n} \nabla f_{\alpha_{n}}\right) p\right\|+\left\|P_{C}\left(I-\lambda_{n} \nabla f_{\alpha_{n}}\right) p-P_{C}\left(I-\lambda_{n} \nabla f\right) p\right\| \\
& \quad \leq\left\|v_{n}-p\right\|+\left\|\left(I-\lambda_{n} \nabla f_{\alpha_{n}}\right) p-\left(I-\lambda_{n} \nabla f\right) p\right\| \\
& \quad=\left\|v_{n}-p\right\|+\lambda_{n} \alpha_{n}\|p\| \\
& \quad \leq\left\|x_{n}-p\right\|+\lambda_{n} \alpha_{n}\|p\| .
\end{aligned}
$$

Since $\left(\gamma_{n}+\sigma_{n}\right) \xi \leq \gamma_{n}$ for all $n \geq 0$ and $T$ is $\xi$-strictly pseudocontractive, utilizing Lemma 2.4 we obtain from (3.1) and (3.5)

$$
\begin{aligned}
\left\|y_{n}-p\right\| & =\left\|\beta_{n} x_{n}+\gamma_{n} t_{n}+\sigma_{n} T t_{n}-p\right\| \\
& =\left\|\beta_{n}\left(x_{n}-p\right)+\gamma_{n}\left(t_{n}-p\right)+\sigma_{n}\left(T t_{n}-p\right)\right\| \\
& \leq \beta_{n}\left\|x_{n}-p\right\|+\left\|\gamma_{n}\left(t_{n}-p\right)+\sigma_{n}\left(T t_{n}-p\right)\right\| \\
& \leq \beta_{n}\left\|x_{n}-p\right\|+\left(\gamma_{n}+\sigma_{n}\right)\left\|t_{n}-p\right\| \\
& \leq \beta_{n}\left\|x_{n}-p\right\|+\left(\gamma_{n}+\sigma_{n}\right)\left[\left\|x_{n}-p\right\|+\lambda_{n} \alpha_{n}\|p\|\right] \\
& \leq \beta_{n}\left\|x_{n}-p\right\|+\left(\gamma_{n}+\delta_{n}\right)\left\|x_{n}-p\right\|+\lambda_{n} \alpha_{n}\|p\| \\
& =\left\|x_{n}-p\right\|+\lambda_{n} \alpha_{n}\|p\| .
\end{aligned}
$$

Utilizing Lemma 2.7, we deduce from (3.1), (3.6), $\left\{\lambda_{n}\right\} \subset[a, b] \subset\left(0, \frac{2}{L}\right)$, and $0 \leq \gamma<\tau$ that, for all $n \geq 0$,

$$
\begin{aligned}
& \left\|x_{n+1}-p\right\| \\
& \quad=\left\|\epsilon_{n} \gamma\left(\delta_{n} V x_{n}+\left(1-\delta_{n}\right) S x_{n}\right)+\left(I-\epsilon_{n} \mu F\right) y_{n}-p\right\| \\
& \quad=\left\|\epsilon_{n} \gamma\left(\delta_{n} V x_{n}+\left(1-\delta_{n}\right) S x_{n}\right)-\epsilon_{n} \mu F p+\left(I-\epsilon_{n} \mu F\right) y_{n}-\left(I-\epsilon_{n} \mu F\right) p\right\| \\
& \quad \leq\left\|\epsilon_{n} \gamma\left(\delta_{n} V x_{n}+\left(1-\delta_{n}\right) S x_{n}\right)-\epsilon_{n} \mu F p\right\|+\left\|\left(I-\epsilon_{n} \mu F\right) y_{n}-\left(I-\epsilon_{n} \mu F\right) p\right\| \\
& =\epsilon_{n}\left\|\delta_{n}\left(\gamma V x_{n}-\mu F p\right)+\left(1-\delta_{n}\right)\left(\gamma S x_{n}-\mu F p\right)\right\|+\left\|\left(I-\epsilon_{n} \mu F\right) y_{n}-\left(I-\epsilon_{n} \mu F\right) p\right\| \\
& \quad \leq \epsilon_{n}\left[\delta_{n}\left\|\gamma V x_{n}-\mu F p\right\|+\left(1-\delta_{n}\right)\left\|\gamma S x_{n}-\mu F p\right\|\right]+\left\|\left(I-\epsilon_{n} \mu F\right) y_{n}-\left(I-\epsilon_{n} \mu F\right) p\right\|
\end{aligned}
$$




$$
\begin{aligned}
\leq & \epsilon_{n}\left[\delta_{n}\left(\gamma\left\|V x_{n}-V p\right\|+\|\gamma V p-\mu F p\|\right)+\left(1-\delta_{n}\right)\left(\gamma\left\|S x_{n}-S p\right\|+\|\gamma S p-\mu F p\|\right)\right] \\
& +\left\|\left(I-\epsilon_{n} \mu F\right) y_{n}-\left(I-\lambda_{n} \mu F\right) p\right\| \\
\leq & \epsilon_{n}\left[\delta_{n}\left(\gamma \rho\left\|x_{n}-p\right\|+\|\gamma V p-\mu F p\|\right)+\left(1-\delta_{n}\right)\left(\gamma\left\|x_{n}-p\right\|+\|\gamma S p-\mu F p\|\right)\right] \\
& +\left(1-\epsilon_{n} \tau\right)\left\|y_{n}-p\right\| \\
\leq & \epsilon_{n}\left[\left(1-\delta_{n}(1-\rho)\right) \gamma\left\|x_{n}-p\right\|+\max \{\|\gamma V p-\mu F p\|,\|\gamma S p-\mu F p\|\}\right] \\
& +\left(1-\epsilon_{n} \tau\right)\left[\left\|x_{n}-p\right\|+\lambda_{n} \alpha_{n}\|p\|\right] \\
= & \epsilon_{n}\left(1-\delta_{n}(1-\rho)\right) \gamma\left\|x_{n}-p\right\|+\lambda \max \{\|\gamma V p-\mu F p\|,\|\gamma S p-\mu F p\|\} \\
& +\left(1-\epsilon_{n} \tau\right)\left\|x_{n}-p\right\|+\lambda_{n} \alpha_{n}\|p\| \\
\leq & \epsilon_{n} \gamma\left\|x_{n}-p\right\|+\epsilon_{n} \max \{\|\gamma V p-\mu F p\|,\|\gamma S p-\mu F p\|\}+\left(1-\epsilon_{n} \tau\right)\left\|x_{n}-p\right\| \\
= & \left(1-\epsilon_{n}(\tau-\gamma)\right)\left\|x_{n}-p\right\|+\epsilon_{n} \max \{\|\gamma V p-\mu F p\|,\|\gamma S p-\mu F p\|\}+\alpha_{n} b\|p\| \\
= & \left(1-\epsilon_{n}(\tau-\gamma)\right)\left\|x_{n}-p\right\|+\epsilon_{n}(\tau-\gamma) \frac{\max \{\|\gamma V p-\mu F p\|,\|\gamma S p-\mu F p\|\}}{\tau-\alpha}+\alpha_{n} b\|p\| \\
\leq & \max \left\{\left\|x_{n}-p\right\|, \frac{\|\gamma V p-\mu F p\|}{\tau-\gamma}, \frac{\|\gamma S p-\mu F p\|}{\tau-\gamma}\right\}+\alpha_{n} b\|p\| .
\end{aligned}
$$

By induction, we get

$$
\left\|x_{n+1}-p\right\| \leq \max \left\{\left\|x_{0}-p\right\|, \frac{\|\gamma V p-\mu F p\|}{\tau-\gamma}, \frac{\|\gamma S p-\mu F p\|}{\tau-\gamma}\right\}+\sum_{j=0}^{n} \alpha_{n} b\|p\|, \quad \forall n \geq 0
$$

Thus, $\left\{x_{n}\right\}$ is bounded (due to $\sum_{n=0}^{\infty} \alpha_{n}<\infty$ ) and so are the sequences $\left\{t_{n}\right\},\left\{u_{n}\right\},\left\{v_{n}\right\}$ and $\left\{y_{n}\right\}$.

Step 2. We prove that $\lim _{n \rightarrow \infty} \frac{\left\|x_{n+1}-x_{n}\right\|}{\delta_{n}}=0$.

Indeed, utilizing (2.1) and (2.3), we obtain

$$
\begin{aligned}
&\left\|v_{n+1}-v_{n}\right\| \\
&=\left\|\Lambda_{n+1}^{N} u_{n+1}-\Lambda_{n}^{N} u_{n}\right\| \\
&=\left\|J_{R_{N}, \lambda_{N, n+1}}\left(I-\lambda_{N, n+1} B_{N}\right) \Lambda_{n+1}^{N-1} u_{n+1}-J_{R_{N}, \lambda_{N, n}}\left(I-\lambda_{N, n} B_{N}\right) \Lambda_{n}^{N-1} u_{n}\right\| \\
& \leq\left\|J_{R_{N}, \lambda_{N, n+1}}\left(I-\lambda_{N, n+1} B_{N}\right) \Lambda_{n+1}^{N-1} u_{n+1}-J_{R_{N}, \lambda_{N, n+1}}\left(I-\lambda_{N, n} B_{N}\right) \Lambda_{n+1}^{N-1} u_{n+1}\right\| \\
& \quad+\left\|J_{R_{N}, \lambda_{N, n+1}}\left(I-\lambda_{N, n} B_{N}\right) \Lambda_{n+1}^{N-1} u_{n+1}-J_{R_{N}, \lambda_{N, n}}\left(I-\lambda_{N, n} B_{N}\right) \Lambda_{n}^{N-1} u_{n}\right\| \\
& \leq\left\|\left(I-\lambda_{N, n+1} B_{N}\right) \Lambda_{n+1}^{N-1} u_{n+1}-\left(I-\lambda_{N, n} B_{N}\right) \Lambda_{n+1}^{N-1} u_{n+1}\right\| \\
& \quad+\left\|\left(I-\lambda_{N, n} B_{N}\right) \Lambda_{n+1}^{N-1} u_{n+1}-\left(I-\lambda_{N, n} B_{N}\right) \Lambda_{n}^{N-1} u_{n}\right\|+\left|\lambda_{N, n+1}-\lambda_{N, n}\right| \\
& \quad \times\left(\frac{1}{\lambda_{N, n+1}}\left\|J_{R_{N}, \lambda_{N, n+1}}\left(I-\lambda_{N, n} B_{N}\right) \Lambda_{n+1}^{N-1} u_{n+1}-\left(I-\lambda_{N, n} B_{N}\right) \Lambda_{n}^{N-1} u_{n}\right\|\right. \\
&\left.\quad+\frac{1}{\lambda_{N, n}}\left\|\left(I-\lambda_{N, n} B_{N}\right) \Lambda_{n+1}^{N-1} u_{n+1}-J_{R_{N}, \lambda_{N, n}}\left(I-\lambda_{N, n} B_{N}\right) \Lambda_{n}^{N-1} u_{n}\right\|\right) \\
& \leq\left|\lambda_{N, n+1}-\lambda_{N, n}\right|\left(\left\|B_{N} \Lambda_{n+1}^{N-1} u_{n+1}\right\|+\tilde{M}\right)+\left\|\Lambda_{n+1}^{N-1} u_{n+1}-\Lambda_{n}^{N-1} u_{n}\right\| \\
& \leq \mid \lambda_{N, n+1}-\lambda_{N, n} \mid\left(\left\|B_{N} \Lambda_{n+1}^{N-1} u_{n+1}\right\|+\tilde{M}\right)
\end{aligned}
$$




$$
\begin{aligned}
& +\left|\lambda_{N-1, n+1}-\lambda_{N-1, n}\right|\left(\left\|B_{N-1} \Lambda_{n+1}^{N-2} u_{n+1}\right\|+\tilde{M}\right)+\left\|\Lambda_{n+1}^{N-2} u_{n+1}-\Lambda_{n}^{N-2} u_{n}\right\| \\
& \quad \ldots \\
\leq & \left|\lambda_{N, n+1}-\lambda_{N, n}\right|\left(\left\|B_{N} \Lambda_{n+1}^{N-1} u_{n+1}\right\|+\tilde{M}\right) \\
& +\left|\lambda_{N-1, n+1}-\lambda_{N-1, n}\right|\left(\left\|B_{N-1} \Lambda_{n+1}^{N-2} u_{n+1}\right\|+\tilde{M}\right) \\
& +\cdots+\left|\lambda_{1, n+1}-\lambda_{1, n}\right|\left(\left\|B_{1} \Lambda_{n+1}^{0} u_{n+1}\right\|+\tilde{M}\right)+\left\|\Lambda_{n+1}^{0} u_{n+1}-\Lambda_{n}^{0} u_{n}\right\| \\
& +\tilde{M}_{0} \sum_{i=1}^{N}\left|\lambda_{i, n+1}-\lambda_{i, n}\right|+\left\|u_{n+1}-u_{n}\right\|,
\end{aligned}
$$

where

$$
\begin{aligned}
& \sup _{n \geq 0}\left\{\frac{1}{\lambda_{N, n+1}}\left\|J_{R_{N}, \lambda_{N, n+1}}\left(I-\lambda_{N, n} B_{N}\right) \Lambda_{n+1}^{N-1} u_{n+1}-\left(I-\lambda_{N, n} B_{N}\right) \Lambda_{n}^{N-1} u_{n}\right\|\right. \\
& \left.\quad+\frac{1}{\lambda_{N, n}}\left\|\left(I-\lambda_{N, n} B_{N}\right) \Lambda_{n+1}^{N-1} u_{n+1}-J_{R_{N}, \lambda_{N, n}}\left(I-\lambda_{N, n} B_{N}\right) \Lambda_{n}^{N-1} u_{n}\right\|\right\} \leq \tilde{M}
\end{aligned}
$$

for some $\tilde{M}>0$ and $\sup _{n \geq 0}\left\{\sum_{i=1}^{N}\left\|B_{i} \Lambda_{n+1}^{i-1} u_{n+1}\right\|+\tilde{M}\right\} \leq \tilde{M}_{0}$ for some $\widetilde{M}_{0}>0$.

Utilizing Proposition 2.2(ii), (v), we deduce that

$$
\begin{aligned}
& \left\|u_{n+1}-u_{n}\right\| \\
& =\left\|\Delta_{n+1}^{M} x_{n+1}-\Delta_{n}^{M} x_{n}\right\|
\end{aligned}
$$

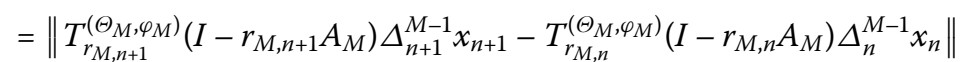

$$
\begin{aligned}
& \leq\left\|T_{r_{M, n+1}}^{\left(\Theta_{M}, \varphi_{M}\right)}\left(I-r_{M, n+1} A_{M}\right) \Delta_{n+1}^{M-1} x_{n+1}-T_{r_{M, n}}^{\left(\Theta_{M}, \varphi_{M}\right)}\left(I-r_{M, n} A_{M}\right) \Delta_{n+1}^{M-1} x_{n+1}\right\| \\
& +\left\|T_{r_{M, n}}^{\left(\Theta_{M}, \varphi_{M}\right)}\left(I-r_{M, n} A_{M}\right) \Delta_{n+1}^{M-1} x_{n+1}-T_{r_{M, n}}^{\left(\Theta_{M}, \varphi_{M}\right)}\left(I-r_{M, n} A_{M}\right) \Delta_{n}^{M-1} x_{n}\right\|
\end{aligned}
$$

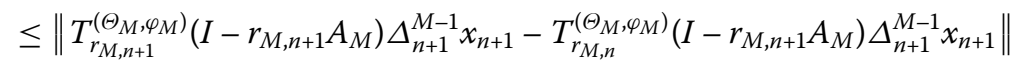

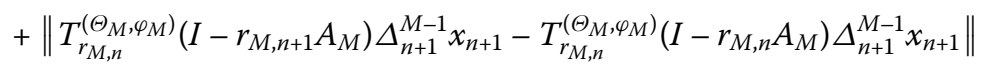

$$
\begin{aligned}
& +\left\|\left(I-r_{M, n} A_{M}\right) \Delta_{n+1}^{M-1} x_{n+1}-\left(I-r_{M, n} A_{M}\right) \Delta_{n}^{M-1} x_{n}\right\| \\
& \leq \frac{\left|r_{M, n+1}-r_{M, n}\right|}{r_{M, n+1}}\left\|T_{r_{M, n+1}}^{\left(\Theta_{\left.M, \varphi_{M}\right)}\right.}\left(I-r_{M, n+1} A_{M}\right) \Delta_{n+1}^{M-1} x_{n+1}-\left(I-r_{M, n+1} A_{M}\right) \Delta_{n+1}^{M-1} x_{n+1}\right\| \\
& +\left|r_{M, n+1}-r_{M, n}\right|\left\|A_{M} \Delta_{n+1}^{M-1} x_{n+1}\right\|+\left\|\Delta_{n+1}^{M-1} x_{n+1}-\Delta_{n}^{M-1} x_{n}\right\|
\end{aligned}
$$

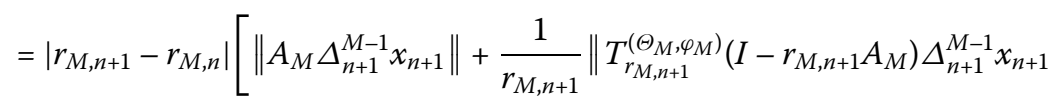

$$
\begin{aligned}
& \left.-\left(I-r_{M, n+1} A_{M}\right) \Delta_{n+1}^{M-1} x_{n+1} \|\right]+\left\|\Delta_{n+1}^{M-1} x_{n+1}-\Delta_{n}^{M-1} x_{n}\right\|
\end{aligned}
$$

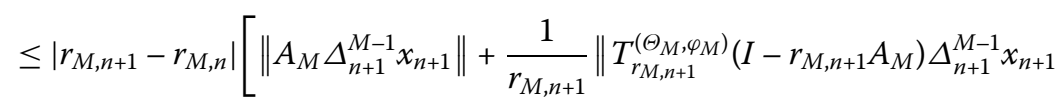

$$
\begin{aligned}
& \left.-\left(I-r_{M, n+1} A_{M}\right) \Delta_{n+1}^{M-1} x_{n+1} \|\right]+\cdots+\left|r_{1, n+1}-r_{1, n}\right|\left[\left\|A_{1} \Delta_{n+1}^{0} x_{n+1}\right\|\right. \\
& \left.+\frac{1}{r_{1, n+1}}\left\|T_{r_{1, n+1}}^{\left(\Theta_{1}, \varphi_{1}\right)}\left(I-r_{1, n+1} A_{1}\right) \Delta_{n+1}^{0} x_{n+1}-\left(I-r_{1, n+1} A_{1}\right) \Delta_{n+1}^{0} x_{n+1}\right\|\right]
\end{aligned}
$$




$$
\begin{gathered}
+\left\|\Delta_{n+1}^{0} x_{n+1}-\Delta_{n}^{0} x_{n}\right\| \\
\leq \widetilde{M}_{1} \sum_{k=1}^{M}\left|r_{k, n+1}-r_{k, n}\right|+\left\|x_{n+1}-x_{n}\right\|,
\end{gathered}
$$

where $\widetilde{M}_{1}>0$ is a constant such that, for each $n \geq 0$,

$$
\begin{aligned}
& \sum_{k=1}^{M}\left[\left\|A_{k} \Delta_{n+1}^{k-1} x_{n+1}\right\|+\frac{1}{r_{k, n+1}}\left\|T_{r_{k, n+1}}^{\left(\Theta_{k}, \varphi_{k}\right)}\left(I-r_{k, n+1} A_{k}\right) \Delta_{n+1}^{k-1} x_{n+1}-\left(I-r_{k, n+1} A_{k}\right) \Delta_{n+1}^{k-1} x_{n+1}\right\|\right] \\
& \quad \leq \tilde{M}_{1} .
\end{aligned}
$$

Furthermore, we define $y_{n}=\beta_{n} x_{n}+\left(1-\beta_{n}\right) w_{n}$ for all $n \geq 0$. It follows that

$$
\begin{aligned}
& w_{n+1}-w_{n} \\
&= \frac{y_{n+1}-\beta_{n+1} x_{n+1}}{1-\beta_{n+1}}-\frac{y_{n}-\beta_{n} x_{n}}{1-\beta_{n}} \\
&= \frac{\gamma_{n+1} t_{n+1}+\sigma_{n+1} T t_{n+1}}{1-\beta_{n+1}}-\frac{\gamma_{n} t_{n}+\sigma_{n} T t_{n}}{1-\beta_{n}} \\
&= \frac{\gamma_{n+1}\left(t_{n+1}-t_{n}\right)+\sigma_{n+1}\left(T t_{n+1}-T t_{n}\right)}{1-\beta_{n+1}} \\
& \quad+\left(\frac{\gamma_{n+1}}{1-\beta_{n+1}}-\frac{\gamma_{n}}{1-\beta_{n}}\right) t_{n}+\left(\frac{\sigma_{n+1}}{1-\beta_{n+1}}-\frac{\sigma_{n}}{1-\beta_{n}}\right) T t_{n} .
\end{aligned}
$$

Since $\left(\gamma_{n}+\sigma_{n}\right) \xi \leq \gamma_{n}$ for all $n \geq 0$, utilizing Lemma 2.4 and the nonexpansivity of $P_{C}(I-$ $\left.\lambda_{n} \nabla f_{\alpha_{n}}\right)$ we have

$$
\left\|\gamma_{n+1}\left(t_{n+1}-t_{n}\right)+\sigma_{n+1}\left(T t_{n+1}-T t_{n}\right)\right\| \leq\left(\gamma_{n+1}+\sigma_{n+1}\right)\left\|t_{n+1}-t_{n}\right\|
$$

and

$$
\begin{aligned}
\left\|t_{n+1}-t_{n}\right\|= & \left\|P_{C}\left(I-\lambda_{n+1} \nabla f_{\alpha_{n+1}}\right) v_{n+1}-P_{C}\left(I-\lambda_{n} \nabla f_{\alpha_{n}}\right) v_{n}\right\| \\
\leq & \left\|P_{C}\left(I-\lambda_{n+1} \nabla f_{\alpha_{n+1}}\right) v_{n+1}-P_{C}\left(I-\lambda_{n+1} \nabla f_{\alpha_{n+1}}\right) v_{n}\right\| \\
& \quad\left\|P_{C}\left(I-\lambda_{n+1} \nabla f_{\alpha_{n+1}}\right) v_{n}-P_{C}\left(I-\lambda_{n} \nabla f_{\alpha_{n}}\right) v_{n}\right\| \\
\leq & \left\|v_{n+1}-v_{n}\right\|+\left\|\left(I-\lambda_{n+1} \nabla f_{\alpha_{n+1}}\right) v_{n}-\left(I-\lambda_{n} \nabla f_{\alpha_{n}}\right) v_{n}\right\| \\
\leq & \left\|v_{n+1}-v_{n}\right\|+\left|\lambda_{n+1} \alpha_{n+1}-\lambda_{n} \alpha_{n}\right|\left\|v_{n}\right\|+\left|\lambda_{n+1}-\lambda_{n}\right|\left\|\nabla f\left(v_{n}\right)\right\| .
\end{aligned}
$$

Hence it follows from (3.7)-(3.11) that

$$
\begin{aligned}
&\left\|w_{n+1}-w_{n}\right\| \\
& \leq \frac{\left\|\gamma_{n+1}\left(t_{n+1}-t_{n}\right)+\sigma_{n+1}\left(T t_{n+1}-T t_{n}\right)\right\|}{1-\beta_{n+1}} \\
&+\left|\frac{\gamma_{n+1}}{1-\beta_{n+1}}-\frac{\gamma_{n}}{1-\beta_{n}}\right|\left\|t_{n}\right\|+\left|\frac{\sigma_{n+1}}{1-\beta_{n+1}}-\frac{\sigma_{n}}{1-\beta_{n}}\right|\left\|T t_{n}\right\| \\
& \leq \frac{\left(\gamma_{n+1}+\sigma_{n+1}\right)}{1-\beta_{n+1}}\left\|t_{n+1}-t_{n}\right\|+\left|\frac{\gamma_{n+1}}{1-\beta_{n+1}}-\frac{\gamma_{n}}{1-\beta_{n}}\right|\left(\left\|t_{n}\right\|+\left\|T t_{n}\right\|\right)
\end{aligned}
$$




$$
\begin{aligned}
= & \left\|t_{n+1}-t_{n}\right\|+\left|\frac{\gamma_{n+1}}{1-\beta_{n+1}}-\frac{\gamma_{n}}{1-\beta_{n}}\right|\left(\left\|t_{n}\right\|+\left\|T t_{n}\right\|\right) \\
\leq & \left\|v_{n+1}-v_{n}\right\|+\left|\lambda_{n+1} \alpha_{n+1}-\lambda_{n} \alpha_{n}\right|\left\|v_{n}\right\|+\left|\lambda_{n+1}-\lambda_{n}\right|\left\|\nabla f\left(v_{n}\right)\right\| \\
& +\left|\frac{\gamma_{n+1}}{1-\beta_{n+1}}-\frac{\gamma_{n}}{1-\beta_{n}}\right|\left(\left\|t_{n}\right\|+\left\|T t_{n}\right\|\right) \\
\leq & \tilde{M}_{0} \sum_{i=1}^{N}\left|\lambda_{i, n+1}-\lambda_{i, n}\right|+\left\|u_{n+1}-u_{n}\right\|+\left|\lambda_{n+1} \alpha_{n+1}-\lambda_{n} \alpha_{n}\right|\left\|v_{n}\right\| \\
& +\left|\lambda_{n+1}-\lambda_{n}\right|\left\|\left|\nabla f\left(v_{n}\right) \|+\right| \frac{\gamma_{n+1}}{1-\beta_{n+1}}-\frac{\gamma_{n}}{1-\beta_{n}} \mid\left(\left\|t_{n}\right\|+\left\|T t_{n}\right\|\right)\right. \\
\leq & \tilde{M}_{0} \sum_{i=1}^{N}\left|\lambda_{i, n+1}-\lambda_{i, n}\right|+\tilde{M}_{1} \sum_{k=1}^{M}\left|r_{k, n+1}-r_{k, n}\right|+\left\|x_{n+1}-x_{n}\right\| \\
& +\left|\lambda_{n+1} \alpha_{n+1}-\lambda_{n} \alpha_{n}\right|\left\|v_{n}\right\|+\left|\lambda_{n+1}-\lambda_{n}\right|\left\|\nabla f\left(v_{n}\right)\right\| \\
& +\left|\frac{\gamma_{n+1}}{1-\beta_{n+1}}-\frac{\gamma_{n}}{1-\beta_{n}}\right|\left(\left\|t_{n}\right\|+\left\|T t_{n}\right\|\right) .
\end{aligned}
$$

In the meantime, a simple calculation shows that

$$
y_{n+1}-y_{n}=\beta_{n}\left(x_{n+1}-x_{n}\right)+\left(1-\beta_{n}\right)\left(w_{n+1}-w_{n}\right)+\left(\beta_{n+1}-\beta_{n}\right)\left(x_{n+1}-w_{n+1}\right) .
$$

So, it follows from (3.12) that

$$
\begin{aligned}
& \left\|y_{n+1}-y_{n}\right\| \\
& \leq \beta_{n}\left\|x_{n+1}-x_{n}\right\|+\left(1-\beta_{n}\right)\left\|w_{n+1}-w_{n}\right\|+\left|\beta_{n+1}-\beta_{n}\right|\left\|x_{n+1}-w_{n+1}\right\| \\
& \leq \beta_{n}\left\|x_{n+1}-x_{n}\right\|+\left(1-\beta_{n}\right)\left[\tilde{M}_{0} \sum_{i=1}^{N}\left|\lambda_{i, n+1}-\lambda_{i, n}\right|+\tilde{M}_{1} \sum_{k=1}^{M}\left|r_{k, n+1}-r_{k, n}\right|\right. \\
& +\left\|x_{n+1}-x_{n}\right\|+\left|\frac{\gamma_{n+1}}{1-\beta_{n+1}}-\frac{\gamma_{n}}{1-\beta_{n}}\right|\left(\left\|t_{n}\right\|+\left\|T t_{n}\right\|\right)+\left|\lambda_{n+1} \alpha_{n+1}-\lambda_{n} \alpha_{n}\right|\left\|v_{n}\right\| \\
& \left.+\left|\lambda_{n+1}-\lambda_{n}\right|\left\|\nabla f\left(v_{n}\right)\right\|\right]+\left|\beta_{n+1}-\beta_{n}\right||| x_{n+1}-w_{n+1}|| \\
& \leq\left\|x_{n+1}-x_{n}\right\|+\widetilde{M}_{0} \sum_{i=1}^{N}\left|\lambda_{i, n+1}-\lambda_{i, n}\right|+\widetilde{M}_{1} \sum_{k=1}^{M}\left|r_{k, n+1}-r_{k, n}\right| \\
& +\frac{\left|\gamma_{n+1}-\gamma_{n}\right|\left(1-\beta_{n}\right)+\gamma_{n}\left|\beta_{n+1}-\beta_{n}\right|}{1-\beta_{n+1}}\left(\left\|t_{n}\right\|+\left\|T t_{n}\right\|\right)+\left|\lambda_{n+1} \alpha_{n+1}-\lambda_{n} \alpha_{n}\right|\left\|v_{n}\right\| \\
& +\left|\lambda_{n+1}-\lambda_{n}\right|\left\|\nabla f\left(v_{n}\right)\right\|+\left|\beta_{n+1}-\beta_{n}\right|\left\|x_{n+1}-w_{n+1}\right\| \\
& \leq\left\|x_{n+1}-x_{n}\right\|+\tilde{M}_{0} \sum_{i=1}^{N}\left|\lambda_{i, n+1}-\lambda_{i, n}\right|+\widetilde{M}_{1} \sum_{k=1}^{M}\left|r_{k, n+1}-r_{k, n}\right| \\
& +\left|\gamma_{n+1}-\gamma_{n}\right| \frac{\left\|t_{n}\right\|+\left\|T t_{n}\right\|}{1-d}+\left|\beta_{n+1}-\beta_{n}\right|\left(\left\|x_{n+1}-w_{n+1}\right\|+\frac{\left\|t_{n}\right\|+\left\|T t_{n}\right\|}{1-d}\right) \\
& +\left|\lambda_{n+1} \alpha_{n+1}-\lambda_{n} \alpha_{n}\right|\left\|v_{n}\right\|+\left|\lambda_{n+1}-\lambda_{n}\right|\left\|\nabla f\left(v_{n}\right)\right\|
\end{aligned}
$$




$$
\begin{aligned}
& \leq|| x_{n+1}-x_{n} \|+\tilde{M}_{2}\left(\sum_{i=1}^{N}\left|\lambda_{i, n+1}-\lambda_{i, n}\right|+\sum_{k=1}^{M}\left|r_{k, n+1}-r_{k, n}\right|+\left|\gamma_{n+1}-\gamma_{n}\right|\right. \\
& \left.\quad+\left|\beta_{n+1}-\beta_{n}\right|+\left|\lambda_{n+1} \alpha_{n+1}-\lambda_{n} \alpha_{n}\right|+\left|\lambda_{n+1}-\lambda_{n}\right|\right)
\end{aligned}
$$

where $\sup _{n \geq 0}\left\{\left\|x_{n+1}-w_{n+1}\right\|+\frac{\left\|t_{n}\right\|+\left\|T t_{n}\right\|}{1-d}+\left\|v_{n}\right\|+\left\|\nabla f\left(v_{n}\right)\right\|+\widetilde{M}_{0}+\widetilde{M}_{1}\right\} \leq \widetilde{M}_{2}$ for some $\tilde{M}_{2}>0$.

On the other hand, we define $z_{n}:=\delta_{n} V x_{n}+\left(1-\delta_{n}\right) S x_{n}$ for all $n \geq 0$. Then it is well known that $x_{n+1}=\epsilon_{n} \gamma z_{n}+\left(I-\epsilon_{n} \mu F\right) y_{n}$ for all $n \geq 0$. Simple calculations show that

$$
\left\{\begin{aligned}
z_{n+1}-z_{n}= & \left(\delta_{n+1}-\delta_{n}\right)\left(V x_{n}-S x_{n}\right)+\delta_{n+1}\left(V x_{n+1}-V x_{n}\right) \\
+ & \left(1-\delta_{n+1}\right)\left(S x_{n+1}-S x_{n}\right) \\
x_{n+2}-x_{n+1}= & \left(\epsilon_{n+1}-\epsilon_{n}\right)\left(\gamma z_{n}-\mu F y_{n}\right)+\epsilon_{n+1} \gamma\left(z_{n+1}-z_{n}\right) \\
& +\left(I-\lambda_{n+1} \mu F\right) y_{n+1}-\left(I-\lambda_{n+1} \mu F\right) y_{n} .
\end{aligned}\right.
$$

Since $V$ is a $\rho$-contraction with coefficient $\rho \in[0,1)$ and $S$ is a nonexpansive mapping, we conclude that

$$
\begin{aligned}
\left\|z_{n+1}-z_{n}\right\| & \leq\left|\delta_{n+1}-\delta_{n}\right|\left\|V x_{n}-S x_{n}\right\|+\delta_{n+1}\left\|V x_{n+1}-V x_{n}\right\|+\left(1-\delta_{n+1}\right)\left\|S x_{n+1}-S x_{n}\right\| \\
& \leq\left|\delta_{n+1}-\delta_{n}\right|\left\|V x_{n}-S x_{n}\right\|+\delta_{n+1} \rho\left\|x_{n+1}-x_{n}\right\|+\left(1-\delta_{n+1}\right)\left\|x_{n+1}-x_{n}\right\| \\
& =\left(1-\delta_{n+1}(1-\rho)\right)\left\|x_{n+1}-x_{n}\right\|+\left|\delta_{n+1}-\delta_{n}\right|\left\|V x_{n}-S x_{n}\right\|,
\end{aligned}
$$

which, together with (3.13) and $0 \leq \gamma<\tau$, implies that

$$
\begin{aligned}
& \left\|x_{n+2}-x_{n+1}\right\| \\
& \leq\left|\epsilon_{n+1}-\epsilon_{n}\right|\left\|\gamma z_{n}-\mu F y_{n}\right\|+\epsilon_{n+1} \gamma\left\|z_{n+1}-z_{n}\right\| \\
& +\left\|\left(I-\epsilon_{n+1} \mu F\right) y_{n+1}-\left(I-\epsilon_{n+1} \mu F\right) y_{n}\right\| \\
& \leq\left|\epsilon_{n+1}-\epsilon_{n}\right|\left\|\gamma z_{n}-\mu F y_{n}\right\|+\epsilon_{n+1} \gamma\left\|z_{n+1}-z_{n}\right\|+\left(1-\epsilon_{n+1} \tau\right)\left\|y_{n+1}-y_{n}\right\| \\
& \leq\left|\epsilon_{n+1}-\epsilon_{n}\right|\left\|\gamma z_{n}-\mu F y_{n}\right\|+\epsilon_{n+1} \gamma\left[\left(1-\delta_{n+1}(1-\rho)\right)\left\|x_{n+1}-x_{n}\right\|\right. \\
& \left.+\left|\delta_{n+1}-\delta_{n}\right|\left\|V x_{n}-S x_{n}\right\|\right]+\left(1-\epsilon_{n+1} \tau\right)\left[\left\|x_{n+1}-x_{n}\right\|+\tilde{M}_{2}\left(\sum_{i=1}^{N}\left|\lambda_{i, n+1}-\lambda_{i, n}\right|\right.\right. \\
& +\sum_{k=1}^{M}\left|r_{k, n+1}-r_{k, n}\right|+\left|\gamma_{n+1}-\gamma_{n}\right|+\left|\beta_{n+1}-\beta_{n}\right| \\
& \left.\left.+\left|\lambda_{n+1} \alpha_{n+1}-\lambda_{n} \alpha_{n}\right|+\left|\lambda_{n+1}-\lambda_{n}\right|\right)\right] \\
& \leq\left(1-\epsilon_{n+1}(\tau-\gamma)\right)\left\|x_{n+1}-x_{n}\right\|+\left|\epsilon_{n+1}-\epsilon_{n}\right|\left\|\gamma z_{n}-\mu F y_{n}\right\|+\left|\delta_{n+1}-\delta_{n}\right|\left\|V x_{n}-S x_{n}\right\| \\
& +\tilde{M}_{2}\left(\sum_{i=1}^{N}\left|\lambda_{i, n+1}-\lambda_{i, n}\right|+\sum_{k=1}^{M}\left|r_{k, n+1}-r_{k, n}\right|+\left|\gamma_{n+1}-\gamma_{n}\right|+\left|\beta_{n+1}-\beta_{n}\right|\right. \\
& \left.+\left|\lambda_{n+1} \alpha_{n+1}-\lambda_{n} \alpha_{n}\right|+\left|\lambda_{n+1}-\lambda_{n}\right|\right) \\
& \leq\left(1-\epsilon_{n+1}(\tau-\gamma)\right)\left\|x_{n+1}-x_{n}\right\|
\end{aligned}
$$




$$
\begin{aligned}
& +\widetilde{M}_{3}\left\{\sum_{i=1}^{N}\left|\lambda_{i, n+1}-\lambda_{i, n}\right|+\sum_{k=1}^{M}\left|r_{k, n+1}-r_{k, n}\right|+\left|\epsilon_{n+1}-\epsilon_{n}\right|\right. \\
& \left.+\left|\delta_{n+1}-\delta_{n}\right|+\left|\beta_{n+1}-\beta_{n}\right|+\left|\gamma_{n+1}-\gamma_{n}\right|+\left|\lambda_{n+1} \alpha_{n+1}-\lambda_{n} \alpha_{n}\right|+\left|\lambda_{n+1}-\lambda_{n}\right|\right\},
\end{aligned}
$$

where $\sup _{n \geq 0}\left\{\left\|\gamma z_{n}-\mu F y_{n}\right\|+\left\|V x_{n}-S x_{n}\right\|+\widetilde{M}_{2}\right\} \leq \widetilde{M}_{3}$ for some $\widetilde{M}_{3}>0$. Consequently,

$$
\begin{aligned}
& \frac{\left\|x_{n+1}-x_{n}\right\|}{\delta_{n}} \\
& \leq\left(1-\epsilon_{n}(\tau-\gamma)\right) \frac{|| x_{n}-x_{n-1} \mid}{\delta_{n}}+\widetilde{M}_{3}\left\{\sum_{i=1}^{N} \frac{\left|\lambda_{i, n}-\lambda_{i, n-1}\right|}{\delta_{n}}\right. \\
& \quad+\sum_{k=1}^{M} \frac{\left|r_{k, n}-r_{k, n-1}\right|}{\delta_{n}}+\frac{\left|\epsilon_{n}-\epsilon_{n-1}\right|}{\delta_{n}}+\frac{\left|\delta_{n}-\delta_{n-1}\right|}{\delta_{n}}+\frac{\left|\beta_{n}-\beta_{n-1}\right|}{\delta_{n}} \\
&\left.\quad+\frac{\left|\gamma_{n}-\gamma_{n-1}\right|}{\delta_{n}}+\frac{\left|\lambda_{n+1} \alpha_{n+1}-\lambda_{n} \alpha_{n}\right|}{\delta_{n}}+\frac{\left|\lambda_{n+1}-\lambda_{n}\right|}{\delta_{n}}\right\} \\
&=\left(1-\epsilon_{n}(\tau-\gamma)\right) \frac{\left\|x_{n}-x_{n-1}\right\|}{\delta_{n-1}}+\left(1-\epsilon_{n}(\tau-\gamma)\right) \| x_{n}-x_{n-1}||\left(\frac{1}{\delta_{n}}-\frac{1}{\delta_{n-1}}\right) \\
& \quad+\widetilde{M}_{3}\left\{\sum_{i=1}^{N} \frac{\left|\lambda_{i, n}-\lambda_{i, n-1}\right|}{\delta_{n}}+\sum_{k=1}^{M} \frac{\left|r_{k, n}-r_{k, n-1}\right|}{\delta_{n}}+\frac{\left|\epsilon_{n}-\epsilon_{n-1}\right|}{\delta_{n}}+\frac{\left|\delta_{n}-\delta_{n-1}\right|}{\delta_{n}}\right. \\
&\left.\quad+\frac{\left|\beta_{n}-\beta_{n-1}\right|}{\delta_{n}}+\frac{\left|\gamma_{n}-\gamma_{n-1}\right|}{\delta_{n}}+\frac{\left|\lambda_{n+1} \alpha_{n+1}-\lambda_{n} \alpha_{n}\right|}{\delta_{n}}+\frac{\left|\lambda_{n+1}-\lambda_{n}\right|}{\delta_{n}}\right\} \\
& \leq\left(1-\epsilon_{n}(\tau-\gamma)\right) \frac{|| x_{n}-x_{n-1} \|}{\delta_{n-1}}+\epsilon_{n}(\tau-\gamma) \cdot \frac{\tilde{M}_{4}}{\tau-\gamma}\left\{\frac{1}{\epsilon_{n}}\left|\frac{1}{\delta_{n}}-\frac{1}{\delta_{n-1}}\right|+\sum_{i=1}^{N} \frac{\left|\lambda_{i, n}-\lambda_{i, n-1}\right|}{\epsilon_{n} \delta_{n}}\right. \\
&+\sum_{k=1}^{M} \frac{\left|r_{k, n}-r_{k, n-1}\right|}{\epsilon_{n} \delta_{n}}+\frac{1}{\delta_{n}}\left|1-\frac{\epsilon_{n-1}}{\epsilon_{n}}\right|+\frac{1}{\epsilon_{n}}\left|1-\frac{\delta_{n-1}}{\delta_{n}}\right|+\frac{\left|\beta_{n}-\beta_{n-1}\right|}{\epsilon_{n} \delta_{n}}+\frac{\left|\gamma_{n}-\gamma_{n-1}\right|}{\epsilon_{n} \delta_{n}} \\
&\left.+\frac{\lambda_{n+1} \alpha_{n+1}-\lambda_{n} \alpha_{n} \mid}{\epsilon_{n} \delta_{n}}+\frac{\left|\lambda_{n+1}-\lambda_{n}\right|}{\epsilon_{n} \delta_{n}}\right\},
\end{aligned}
$$

where $\sup _{n \geq 1}\left\{\left\|x_{n}-x_{n-1}\right\|+\widetilde{M}_{3}\right\} \leq \widetilde{M}_{4}$ for some $\widetilde{M}_{4}>0$. From conditions (C1)-(C5) it follows that $\sum_{n=0}^{\infty} \epsilon_{n}(\tau-\gamma)=\infty$ and

$$
\begin{aligned}
\lim _{n \rightarrow \infty} \frac{\tilde{M}_{4}}{\tau-\gamma}\left\{\frac{1}{\epsilon_{n}}\left|\frac{1}{\delta_{n}}-\frac{1}{\delta_{n-1}}\right|+\sum_{i=1}^{N} \frac{\left|\lambda_{i, n}-\lambda_{i, n-1}\right|}{\epsilon_{n} \delta_{n}}+\sum_{k=1}^{M} \frac{\left|r_{k, n}-r_{k, n-1}\right|}{\epsilon_{n} \delta_{n}}+\frac{1}{\delta_{n}}\left|1-\frac{\epsilon_{n-1}}{\epsilon_{n}}\right|\right. \\
\left.+\frac{1}{\epsilon_{n}}\left|1-\frac{\delta_{n-1}}{\delta_{n}}\right|+\frac{\left|\beta_{n}-\beta_{n-1}\right|}{\epsilon_{n} \delta_{n}}+\frac{\left|\gamma_{n}-\gamma_{n-1}\right|}{\epsilon_{n} \delta_{n}}+\frac{\left|\lambda_{n+1} \alpha_{n+1}-\lambda_{n} \alpha_{n}\right|}{\epsilon_{n} \delta_{n}}+\frac{\left|\lambda_{n+1}-\lambda_{n}\right|}{\epsilon_{n} \delta_{n}}\right\}=0 .
\end{aligned}
$$

Thus, utilizing Lemma 2.8, we immediately conclude that

$$
\lim _{n \rightarrow \infty} \frac{\left\|x_{n+1}-x_{n}\right\|}{\delta_{n}}=0
$$


So, from $\delta_{n} \rightarrow 0$ it follows that

$$
\lim _{n \rightarrow \infty}\left\|x_{n+1}-x_{n}\right\|=0
$$

Step 3. We prove that $\lim _{n \rightarrow \infty}\left\|x_{n}-u_{n}\right\|=0, \lim _{n \rightarrow \infty}\left\|x_{n}-v_{n}\right\|=0, \lim _{n \rightarrow \infty}\left\|v_{n}-t_{n}\right\|=0$ and $\lim _{n \rightarrow \infty}\left\|t_{n}-T t_{n}\right\|=0$.

Indeed, utilizing Lemmas 2.1 and 2.2(b), from (3.1), (3.4)-(3.5), and $0 \leq \gamma<\tau$ we deduce that

$$
\begin{aligned}
\| y_{n} & -p \|^{2} \\
& =\left\|\beta_{n} x_{n}+\gamma_{n} t_{n}+\sigma_{n} T t_{n}-p\right\|^{2} \\
& =\left\|\beta_{n}\left(x_{n}-p\right)+\left(1-\beta_{n}\right)\left(\frac{\gamma_{n} t_{n}+\sigma_{n} T t_{n}}{1-\beta_{n}}-p\right)\right\|^{2} \\
& =\beta_{n}\left\|x_{n}-p\right\|^{2}+\left(1-\beta_{n}\right)\left\|\frac{\gamma_{n} t_{n}+\sigma_{n} T t_{n}}{1-\beta_{n}}-p\right\|^{2}-\beta_{n}\left(1-\beta_{n}\right)\left\|\frac{\gamma_{n} t_{n}+\sigma_{n} T t_{n}}{1-\beta_{n}}-x_{n}\right\|^{2} \\
& =\beta_{n}\left\|x_{n}-p\right\|^{2}+\left(1-\beta_{n}\right)\left\|\frac{\gamma_{n}\left(t_{n}-p\right)+\sigma_{n}\left(T t_{n}-p\right)}{1-\beta_{n}}\right\|^{2}-\beta_{n}\left(1-\beta_{n}\right)\left\|\frac{y_{n}-x_{n}}{1-\beta_{n}}\right\|^{2} \\
& \leq \beta_{n}\left\|x_{n}-p\right\|^{2}+\left(1-\beta_{n}\right) \frac{\left(\gamma_{n}+\sigma_{n}\right)^{2}\left\|t_{n}-p\right\|^{2}}{\left(1-\beta_{n}\right)^{2}}-\frac{\beta_{n}}{1-\beta_{n}}\left\|y_{n}-x_{n}\right\|^{2} \\
& =\beta_{n}\left\|x_{n}-p\right\|^{2}+\left(1-\beta_{n}\right)\left\|t_{n}-p\right\|^{2}-\frac{\beta_{n}}{1-\beta_{n}}\left\|y_{n}-x_{n}\right\|^{2} \\
& \leq \beta_{n}\left\|x_{n}-p\right\|^{2}+\left(1-\beta_{n}\right)\left(\left\|x_{n}-p\right\|+\lambda_{n} \alpha_{n}\|p\|\right)^{2}-\frac{\beta_{n}}{1-\beta_{n}}\left\|y_{n}-x_{n}\right\|^{2} \\
& \leq \beta_{n}\left(\left\|x_{n}-p\right\|+\lambda_{n} \alpha_{n}\|p\|\right)^{2}+\left(1-\beta_{n}\right)\left(\left\|x_{n}-p\right\|+\lambda_{n} \alpha_{n}\|p\|\right)^{2}-\frac{\beta_{n}}{1-\beta_{n}}\left\|y_{n}-x_{n}\right\|^{2} \\
& =\left(\left\|x_{n}-p\right\|+\lambda_{n} \alpha_{n}\|p\|\right)^{2}-\frac{\beta_{n}}{1-\beta_{n}}\left\|y_{n}-x_{n}\right\|^{2} \\
& \leq\left(\left\|x_{n}-p\right\|+\alpha_{n} b\|p\|\right)^{2}-\frac{\beta_{n}}{1-\beta_{n}}\left\|y_{n}-x_{n}\right\|^{2},
\end{aligned}
$$

and hence

$$
\begin{aligned}
&\left\|x_{n+1}-p\right\|^{2} \\
&=\left\|\epsilon_{n} \gamma\left(\delta_{n} V x_{n}+\left(1-\delta_{n}\right) S x_{n}\right)+\left(I-\epsilon_{n} \mu F\right) y_{n}-p\right\|^{2} \\
&=\left\|\epsilon_{n} \gamma\left(\delta_{n} V x_{n}+\left(1-\delta_{n}\right) S x_{n}\right)-\epsilon_{n} \mu F p+\left(I-\epsilon_{n} \mu F\right) y_{n}-\left(I-\epsilon_{n} \mu F\right) p\right\|^{2} \\
&=\left\|\epsilon_{n}\left[\delta_{n}\left(\gamma V x_{n}-\mu F p\right)+\left(1-\delta_{n}\right)\left(\gamma S x_{n}-\mu F p\right)\right]+\left(I-\epsilon_{n} \mu F\right) y_{n}-\left(I-\epsilon_{n} \mu F\right) p\right\|^{2} \\
&=\| \epsilon_{n}\left[\delta_{n}\left(\gamma V x_{n}-\gamma V p\right)+\left(1-\delta_{n}\right)\left(\gamma S x_{n}-\gamma S p\right)\right]+\left(I-\epsilon_{n} \mu F\right) y_{n}-\left(I-\epsilon_{n} \mu F\right) p \\
& \quad+\epsilon_{n}\left[\delta_{n}(\gamma V p-\mu F p)+\left(1-\delta_{n}\right)(\gamma S p-\mu F p)\right] \|^{2} \\
& \leq\left\|\epsilon_{n}\left[\delta_{n}\left(\gamma V x_{n}-\gamma V p\right)+\left(1-\delta_{n}\right)\left(\gamma S x_{n}-\gamma S p\right)\right]+\left(I-\epsilon_{n} \mu F\right) y_{n}-\left(I-\epsilon_{n} \mu F\right) p\right\|^{2} \\
& \quad+2 \epsilon_{n} \delta_{n}\left\langle(\gamma V p-\mu F p), x_{n+1}-p\right\rangle+2 \epsilon_{n}\left(1-\delta_{n}\right)\left((\gamma S p-\mu F p), x_{n+1}-p\right\rangle \\
& \leq {\left[\epsilon_{n}\left\|\delta_{n}\left(\gamma V x_{n}-\gamma V p\right)+\left(1-\delta_{n}\right)\left(\gamma S x_{n}-\gamma S p\right)\right\|+\left\|\left(I-\epsilon_{n} \mu F\right) y_{n}-\left(I-\epsilon_{n} \mu F\right) p\right\|\right]^{2} }
\end{aligned}
$$




$$
\begin{aligned}
& +2 \epsilon_{n} \delta_{n}\left\langle(\gamma V p-\mu F p), x_{n+1}-p\right\rangle+2 \epsilon_{n}\left(1-\delta_{n}\right)\left\langle(\gamma S p-\mu F p), x_{n+1}-p\right\rangle \\
& \leq\left[\epsilon_{n}\left(\delta_{n} \gamma \rho\left\|x_{n}-p\right\|+\left(1-\delta_{n}\right) \gamma\left\|x_{n}-p\right\|\right)+\left(1-\epsilon_{n} \tau\right)\left\|y_{n}-p\right\|\right]^{2} \\
& +2 \epsilon_{n} \delta_{n}\left\langle(\gamma V p-\mu F p), x_{n+1}-p\right\rangle+2\left(1-\delta_{n}\right) \epsilon_{n}\left\langle(\gamma S p-\mu F p), x_{n+1}-p\right\rangle \\
& =\left[\epsilon_{n}\left(1-\delta_{n}(1-\rho)\right) \gamma\left\|x_{n}-p\right\|+\left(1-\epsilon_{n} \tau\right)\left\|y_{n}-p\right\|\right]^{2} \\
& +2 \epsilon_{n} \delta_{n}\left\langle(\gamma V p-\mu F p), x_{n+1}-p\right\rangle+2 \epsilon_{n}\left(1-\delta_{n}\right)\left\langle(\gamma S p-\mu F p), x_{n+1}-p\right\rangle \\
& \leq\left[\epsilon_{n} \gamma\left\|x_{n}-p\right\|+\left(1-\epsilon_{n} \tau\right)\left\|y_{n}-p\right\|\right]^{2} \\
& +2 \epsilon_{n} \delta_{n}\left\langle(\gamma V p-\mu F p), x_{n+1}-p\right\rangle+2 \epsilon_{n}\left(1-\delta_{n}\right)\left\langle(\gamma S p-\mu F p), x_{n+1}-p\right\rangle \\
& =\left[\epsilon_{n} \tau \cdot \frac{\gamma}{\tau}\left\|x_{n}-p\right\|+\left(1-\epsilon_{n} \tau\right)\left\|y_{n}-p\right\|\right]^{2} \\
& +2 \epsilon_{n} \delta_{n}\left\langle(\gamma V p-\mu F p), x_{n+1}-p\right\rangle+2 \epsilon_{n}\left(1-\delta_{n}\right)\left\langle(\gamma S p-\mu F p), x_{n+1}-p\right\rangle \\
& \leq \epsilon_{n} \frac{\gamma^{2}}{\tau}\left\|x_{n}-p\right\|^{2}+\left(1-\epsilon_{n} \tau\right)\left\|y_{n}-p\right\|^{2} \\
& +2 \epsilon_{n} \delta_{n}\left\langle(\gamma V p-\mu F p), x_{n+1}-p\right\rangle+2 \epsilon_{n}\left(1-\delta_{n}\right)\left\langle(\gamma S p-\mu F p), x_{n+1}-p\right\rangle \\
& \leq \epsilon_{n} \frac{\gamma^{2}}{\tau}\left\|x_{n}-p\right\|^{2}+\left(1-\epsilon_{n} \tau\right)\left[\left(\left\|x_{n}-p\right\|+\alpha_{n} b\|p\|\right)^{2}-\frac{\beta_{n}}{1-\beta_{n}}\left\|y_{n}-x_{n}\right\|^{2}\right] \\
& +2 \epsilon_{n} \delta_{n}\left\langle(\gamma V p-\mu F p), x_{n+1}-p\right\rangle+2 \epsilon_{n}\left(1-\delta_{n}\right)\left\langle(\gamma S p-\mu F p), x_{n+1}-p\right\rangle \\
& \leq \epsilon_{n} \frac{\gamma^{2}}{\tau}\left(\left\|x_{n}-p\right\|+\alpha_{n} b\|p\|\right)^{2}+\left(1-\epsilon_{n} \tau\right)\left[\left(\left\|x_{n}-p\right\|+\alpha_{n} b\|p\|\right)^{2}\right. \\
& \left.-\frac{\beta_{n}}{1-\beta_{n}}\left\|y_{n}-x_{n}\right\|^{2}\right]+2 \epsilon_{n} \delta_{n}\left\langle(\gamma V p-\mu F p), x_{n+1}-p\right\rangle \\
& +2 \epsilon_{n}\left(1-\delta_{n}\right)\left\langle(\gamma S p-\mu F p), x_{n+1}-p\right\rangle \\
& =\left(1-\epsilon_{n} \frac{\tau^{2}-\gamma^{2}}{\tau}\right)\left(\left\|x_{n}-p\right\|+\alpha_{n} b\|p\|\right)^{2}-\frac{\beta_{n}\left(1-\epsilon_{n} \tau\right)}{1-\beta_{n}}\left\|y_{n}-x_{n}\right\|^{2} \\
& +2 \epsilon_{n} \delta_{n}\left\langle(\gamma V p-\mu F p), x_{n+1}-p\right\rangle+2 \epsilon_{n}\left(1-\delta_{n}\right)\left\langle(\gamma S p-\mu F p), x_{n+1}-p\right\rangle \\
& \leq\left(\left\|x_{n}-p\right\|+\alpha_{n} b\|p\|\right)^{2}-\frac{\beta_{n}\left(1-\epsilon_{n} \tau\right)}{1-\beta_{n}}\left\|y_{n}-x_{n}\right\|^{2} \\
& +2 \epsilon_{n} \delta_{n}\|\gamma V p-\mu F p\|\left\|x_{n+1}-p\right\|+2 \epsilon_{n}\|\gamma S p-\mu F p\|\left\|x_{n+1}-p\right\|,
\end{aligned}
$$

which, together with $\left\{\beta_{n}\right\} \subset[c, d] \subset(0,1)$, immediately yields

$$
\begin{aligned}
& \frac{c\left(1-\epsilon_{n} \tau\right)}{1-c}\left\|y_{n}-x_{n}\right\|^{2} \\
& \leq \frac{\beta_{n}\left(1-\epsilon_{n} \tau\right)}{1-\beta_{n}}\left\|y_{n}-x_{n}\right\|^{2} \\
& \leq \quad\left(\left\|x_{n}-p\right\|+\alpha_{n} b\|p\|\right)^{2}-\left\|x_{n+1}-p\right\|^{2}+2 \epsilon_{n} \delta_{n}\|\gamma V p-\mu F p\|\left\|x_{n+1}-p\right\| \\
& \quad+2 \epsilon_{n}\|\gamma S p-\mu F p\|\left\|x_{n+1}-p\right\| \\
& \leq \quad\left(\left\|x_{n}-x_{n+1}\right\|+\alpha_{n} b\|p\|\right)\left(\left\|x_{n}-p\right\|+\left\|x_{n+1}-p\right\|+\alpha_{n} b\|p\|\right) \\
& \quad+2 \epsilon_{n} \delta_{n}\|\gamma V p-\mu F p\|\left\|x_{n+1}-p\right\|+2 \epsilon_{n}\|\gamma S p-\mu F p\|\left\|x_{n+1}-p\right\| .
\end{aligned}
$$


Since $\epsilon_{n} \rightarrow 0, \delta_{n} \rightarrow 0, \alpha_{n} \rightarrow 0,\left\|x_{n+1}-x_{n}\right\| \rightarrow 0$, and $\left\{x_{n}\right\}$ is bounded, we have

$$
\lim _{n \rightarrow \infty}\left\|y_{n}-x_{n}\right\|=0
$$

Observe that

$$
\begin{aligned}
\left\|\Delta_{n}^{k} x_{n}-p\right\|^{2} & =\left\|T_{r_{k, n}}^{\left(\Theta_{k}, \varphi_{k}\right)}\left(I-r_{k, n} A_{k}\right) \Delta_{n}^{k-1} x_{n}-T_{r_{k, n}}^{\left(\Theta_{k}, \varphi_{k}\right)}\left(I-r_{k, n} A_{k}\right) p\right\|^{2} \\
& \leq\left\|\left(I-r_{k, n} A_{k}\right) \Delta_{n}^{k-1} x_{n}-\left(I-r_{k, n} A_{k}\right) p\right\|^{2} \\
& \leq\left\|\Delta_{n}^{k-1} x_{n}-p\right\|^{2}+r_{k, n}\left(r_{k, n}-2 \mu_{k}\right)\left\|A_{k} \Delta_{n}^{k-1} x_{n}-A_{k} p\right\|^{2} \\
& \leq\left\|x_{n}-p\right\|^{2}+r_{k, n}\left(r_{k, n}-2 \mu_{k}\right)\left\|A_{k} \Delta_{n}^{k-1} x_{n}-A_{k} p\right\|^{2}
\end{aligned}
$$

and

$$
\begin{aligned}
\left\|\Lambda_{n}^{i} u_{n}-p\right\|^{2} & =\left\|J_{R_{i}, \lambda_{i, n}}\left(I-\lambda_{i, n} B_{i}\right) \Lambda_{n}^{i-1} u_{n}-J_{R_{i}, \lambda_{i, n}}\left(I-\lambda_{i, n} B_{i}\right) p\right\|^{2} \\
& \leq\left\|\left(I-\lambda_{i, n} B_{i}\right) \Lambda_{n}^{i-1} u_{n}-\left(I-\lambda_{i, n} B_{i}\right) p\right\|^{2} \\
& \leq\left\|\Lambda_{n}^{i-1} u_{n}-p\right\|^{2}+\lambda_{i, n}\left(\lambda_{i, n}-2 \eta_{i}\right)\left\|B_{i} \Lambda_{n}^{i-1} u_{n}-B_{i} p\right\|^{2} \\
& \leq\left\|u_{n}-p\right\|^{2}+\lambda_{i, n}\left(\lambda_{i, n}-2 \eta_{i}\right)\left\|B_{i} \Lambda_{n}^{i-1} u_{n}-B_{i} p\right\|^{2} \\
& \leq\left\|x_{n}-p\right\|^{2}+\lambda_{i, n}\left(\lambda_{i, n}-2 \eta_{i}\right)\left\|B_{i} \Lambda_{n}^{i-1} u_{n}-B_{i} p\right\|^{2}
\end{aligned}
$$

for $i \in\{1,2, \ldots, N\}$ and $k \in\{1,2, \ldots, M\}$. Combining (3.5), (3.15), (3.18), and (3.19), we get

$$
\begin{aligned}
\left\|y_{n}-p\right\|^{2} \leq & \beta_{n}\left\|x_{n}-p\right\|^{2}+\left(1-\beta_{n}\right)\left\|t_{n}-p\right\|^{2}-\frac{\beta_{n}}{1-\beta_{n}}\left\|y_{n}-x_{n}\right\|^{2} \\
\leq & \beta_{n}\left\|x_{n}-p\right\|^{2}+\left(1-\beta_{n}\right)\left\|t_{n}-p\right\|^{2} \\
\leq & \beta_{n}\left\|x_{n}-p\right\|^{2}+\left(1-\beta_{n}\right)\left(\left\|v_{n}-p\right\|+\lambda_{n} \alpha_{n}\|p\|\right)^{2} \\
\leq & \beta_{n}\left\|x_{n}-p\right\|^{2}+\left(1-\beta_{n}\right)\left(\left\|v_{n}-p\right\|+\alpha_{n} b\|p\|\right)^{2} \\
= & \beta_{n}\left\|x_{n}-p\right\|^{2}+\left(1-\beta_{n}\right)\left[\left\|v_{n}-p\right\|^{2}+\alpha_{n} b\|p\|\left(2\left\|v_{n}-p\right\|+\alpha_{n} b\|p\|\right)\right] \\
\leq & \beta_{n}\left\|x_{n}-p\right\|^{2}+\left(1-\beta_{n}\right)\left\|v_{n}-p\right\|^{2}+\alpha_{n} b\|p\|\left(2\left\|v_{n}-p\right\|+\alpha_{n} b\|p\|\right) \\
\leq & \beta_{n}\left\|x_{n}-p\right\|^{2}+\left(1-\beta_{n}\right)\left\|\Lambda_{n}^{i} u_{n}-p\right\|^{2}+\alpha_{n} b\|p\|\left(2\left\|v_{n}-p\right\|+\alpha_{n} b\|p\|^{2}\right) \\
\leq & \beta_{n}\left\|x_{n}-p\right\|^{2}+\left(1-\beta_{n}\right)\left[\left\|u_{n}-p\right\|^{2}+\lambda_{i, n}\left(\lambda_{i, n}-2 \eta_{i}\right)\left\|B_{i} \Lambda_{n}^{i-1} u_{n}-B_{i} p\right\|^{2}\right] \\
& +\alpha_{n} b\|p\|\left(2\left\|v_{n}-p\right\|+\alpha_{n} b\|p\|\right) \\
\leq & \beta_{n}\left\|x_{n}-p\right\|^{2}+\left(1-\beta_{n}\right)\left[\left\|\Delta_{n}^{k} x_{n}-p\right\|^{2}+\lambda_{i, n}\left(\lambda_{i, n}-2 \eta_{i}\right)\left\|B_{i} \Lambda_{n}^{i-1} u_{n}-B_{i} p\right\|^{2}\right] \\
& +\alpha_{n} b\|p\|\left(2\left\|v_{n}-p\right\|+\alpha_{n} b\|p\|\right) \\
\leq & \beta_{n}\left\|x_{n}-p\right\|^{2}+\left(1-\beta_{n}\right)\left[\left\|x_{n}-p\right\|^{2}+r_{k, n}\left(r_{k, n}-2 \mu_{k}\right)\left\|A_{k} \Delta_{n}^{k-1} x_{n}-A_{k} p\right\|^{2}\right. \\
& \left.+\lambda_{i, n}\left(\lambda_{i, n}-2 \eta_{i}\right)\left\|B_{i} \Lambda_{n}^{i-1} u_{n}-B_{i} p\right\|^{2}\right]+\alpha_{n} b\|p\|\left(2\left\|v_{n}-p\right\|+\alpha_{n} b\|p\|\right) \\
= & \left\|x_{n}-p\right\|^{2}+\left(1-\beta_{n}\right)\left[r_{k, n}\left(r_{k, n}-2 \mu \mu_{k}\right)\left\|A_{k} \Delta_{n}^{k-1} x_{n}-A_{k} p\right\|^{2}\right. \\
& \left.+\lambda_{i, n}\left(\lambda_{i, n}-2 \eta_{i}\right)\left\|B_{i} \Lambda_{n}^{i-1} u_{n}-B_{i} p\right\|^{2}\right] \\
& \left.+2\left\|v_{n}-p\right\|+\alpha_{n} b\|p\|\right) \\
& \\
& \\
&
\end{aligned}
$$


which immediately leads to

$$
\begin{aligned}
& \left(1-\beta_{n}\right)\left[r_{k, n}\left(2 \mu_{k}-r_{k, n}\right)\left\|A_{k} \Delta_{n}^{k-1} x_{n}-A_{k} p\right\|^{2}+\lambda_{i, n}\left(2 \eta_{i}-\lambda_{i, n}\right)\left\|B_{i} \Lambda_{n}^{i-1} u_{n}-B_{i} p\right\|^{2}\right] \\
& \quad \leq\left\|x_{n}-p\right\|^{2}-\left\|y_{n}-p\right\|^{2}+\alpha_{n} b\|p\|\left(2\left\|v_{n}-p\right\|+\alpha_{n} b\|p\|\right) \\
& \quad \leq\left\|x_{n}-y_{n}\right\|\left(\left\|x_{n}-p\right\|+\left\|y_{n}-p\right\|\right)+\alpha_{n} b\|p\|\left(2\left\|v_{n}-p\right\|+\alpha_{n} b\|p\|\right) .
\end{aligned}
$$

Since $\left\|x_{n}-y_{n}\right\| \rightarrow 0, \alpha_{n} \rightarrow 0,\left\{\beta_{n}\right\} \subset[c, d] \subset(0,1),\left\{\lambda_{i, n}\right\} \subset\left[a_{i}, b_{i}\right] \subset\left(0,2 \eta_{i}\right),\left\{r_{k, n}\right\} \subset$ $\left[c_{k}, d_{k}\right] \subset\left(0,2 \mu_{k}\right), i \in\{1,2, \ldots, N\}, k \in\{1,2, \ldots, M\}$, and $\left\{v_{n}\right\},\left\{x_{n}\right\},\left\{y_{n}\right\}$ are bounded sequences, we have

$$
\lim _{n \rightarrow \infty}\left\|A_{k} \Delta_{n}^{k-1} x_{n}-A_{k} p\right\|=0 \quad \text { and } \quad \lim _{n \rightarrow \infty}\left\|B_{i} \Lambda_{n}^{i-1} u_{n}-B_{i} p\right\|=0
$$

for all $k \in\{1,2, \ldots, M\}$ and $i \in\{1,2, \ldots, N\}$.

Furthermore, by Proposition 2.2(ii) and Lemma 2.2(a) we have

$$
\begin{aligned}
&\left\|\Delta_{n}^{k} x_{n}-p\right\|^{2} \\
&=\left\|T_{r_{k, n}}^{\left(\Theta_{k}, \varphi_{k}\right)}\left(I-r_{k, n} A_{k}\right) \Delta_{n}^{k-1} x_{n}-T_{r_{k, n}}^{\left(\Theta_{k}, \varphi_{k}\right)}\left(I-r_{k, n} A_{k}\right) p\right\|^{2} \\
& \leq\left\langle\left(I-r_{k, n} A_{k}\right) \Delta_{n}^{k-1} x_{n}-\left(I-r_{k, n} A_{k}\right) p, \Delta_{n}^{k} x_{n}-p\right\rangle \\
&= \frac{1}{2}\left(\left\|\left(I-r_{k, n} A_{k}\right) \Delta_{n}^{k-1} x_{n}-\left(I-r_{k, n} A_{k}\right) p\right\|^{2}+\left\|\Delta_{n}^{k} x_{n}-p\right\|^{2}\right. \\
&\left.\quad-\left\|\left(I-r_{k, n} A_{k}\right) \Delta_{n}^{k-1} x_{n}-\left(I-r_{k, n} A_{k}\right) p-\left(\Delta_{n}^{k} x_{n}-p\right)\right\|^{2}\right) \\
& \leq \frac{1}{2}\left(\left\|\Delta_{n}^{k-1} x_{n}-p\right\|^{2}+\left\|\Delta_{n}^{k} x_{n}-p\right\|^{2}-\left\|\Delta_{n}^{k-1} x_{n}-\Delta_{n}^{k} x_{n}-r_{k, n}\left(A_{k} \Delta_{n}^{k-1} x_{n}-A_{k} p\right)\right\|^{2}\right),
\end{aligned}
$$

which implies that

$$
\begin{aligned}
&\left\|\Delta_{n}^{k} x_{n}-p\right\|^{2} \\
& \leq\left\|\Delta_{n}^{k-1} x_{n}-p\right\|^{2}-\left\|\Delta_{n}^{k-1} x_{n}-\Delta_{n}^{k} x_{n}-r_{k, n}\left(A_{k} \Delta_{n}^{k-1} x_{n}-A_{k} p\right)\right\|^{2} \\
&=\left\|\Delta_{n}^{k-1} x_{n}-p\right\|^{2}-\left\|\Delta_{n}^{k-1} x_{n}-\Delta_{n}^{k} x_{n}\right\|^{2}-r_{k, n}^{2}\left\|A_{k} \Delta_{n}^{k-1} x_{n}-A_{k} p\right\|^{2} \\
&+2 r_{k, n}\left(\Delta_{n}^{k-1} x_{n}-\Delta_{n}^{k} x_{n}, A_{k} \Delta_{n}^{k-1} x_{n}-A_{k} p\right\rangle \\
& \leq\left\|\Delta_{n}^{k-1} x_{n}-p\right\|^{2}-\left\|\Delta_{n}^{k-1} x_{n}-\Delta_{n}^{k} x_{n}\right\|^{2}+2 r_{k, n}\left\|\Delta_{n}^{k-1} x_{n}-\Delta_{n}^{k} x_{n}\right\|\left\|A_{k} \Delta_{n}^{k-1} x_{n}-A_{k} p\right\| \\
& \leq\left\|x_{n}-p\right\|^{2}-\left\|\Delta_{n}^{k-1} x_{n}-\Delta_{n}^{k} x_{n}\right\|^{2} \\
&+2 r_{k, n}\left\|\Delta_{n}^{k-1} x_{n}-\Delta_{n}^{k} x_{n}\right\|\left\|A_{k} \Delta_{n}^{k-1} x_{n}-A_{k} p\right\| .
\end{aligned}
$$

By Lemma 2.2(a) and Lemma 2.10, we obtain

$$
\begin{aligned}
& \left\|\Lambda_{n}^{i} u_{n}-p\right\|^{2} \\
& \quad=\left\|J_{R_{i}, \lambda_{i, n}}\left(I-\lambda_{i, n} B_{i}\right) \Lambda_{n}^{i-1} u_{n}-J_{R_{i}, \lambda_{i, n}}\left(I-\lambda_{i, n} B_{i}\right) p\right\|^{2} \\
& \quad \leq\left\langle\left(I-\lambda_{i, n} B_{i}\right) \Lambda_{n}^{i-1} u_{n}-\left(I-\lambda_{i, n} B_{i}\right) p, \Lambda_{n}^{i} u_{n}-p\right\rangle \\
& \quad=\frac{1}{2}\left(\left\|\left(I-\lambda_{i, n} B_{i}\right) \Lambda_{n}^{i-1} u_{n}-\left(I-\lambda_{i, n} B_{i}\right) p\right\|^{2}+\left\|\Lambda_{n}^{i} u_{n}-p\right\|^{2}\right.
\end{aligned}
$$




$$
\begin{aligned}
& \left.-\left\|\left(I-\lambda_{i, n} B_{i}\right) \Lambda_{n}^{i-1} u_{n}-\left(I-\lambda_{i, n} B_{i}\right) p-\left(\Lambda_{n}^{i} u_{n}-p\right)\right\|^{2}\right) \\
\leq & \frac{1}{2}\left(\left\|\Lambda_{n}^{i-1} u_{n}-p\right\|^{2}+\left\|\Lambda_{n}^{i} u_{n}-p\right\|^{2}-\left\|\Lambda_{n}^{i-1} u_{n}-\Lambda_{n}^{i} u_{n}-\lambda_{i, n}\left(B_{i} \Lambda_{n}^{i-1} u_{n}-B_{i} p\right)\right\|^{2}\right) \\
\leq & \frac{1}{2}\left(\left\|u_{n}-p\right\|^{2}+\left\|\Lambda_{n}^{i} u_{n}-p\right\|^{2}-\left\|\Lambda_{n}^{i-1} u_{n}-\Lambda_{n}^{i} u_{n}-\lambda_{i, n}\left(B_{i} \Lambda_{n}^{i-1} u_{n}-B_{i} p\right)\right\|^{2}\right) \\
\leq & \frac{1}{2}\left(\left\|x_{n}-p\right\|^{2}+\left\|\Lambda_{n}^{i} u_{n}-p\right\|^{2}-\left\|\Lambda_{n}^{i-1} u_{n}-\Lambda_{n}^{i} u_{n}-\lambda_{i, n}\left(B_{i} \Lambda_{n}^{i-1} u_{n}-B_{i} p\right)\right\|^{2}\right),
\end{aligned}
$$

which immediately leads to

$$
\begin{aligned}
& \left\|\Lambda_{n}^{i} u_{n}-p\right\|^{2} \\
& \quad \leq\left\|x_{n}-p\right\|^{2}-\left\|\Lambda_{n}^{i-1} u_{n}-\Lambda_{n}^{i} u_{n}-\lambda_{i, n}\left(B_{i} \Lambda_{n}^{i-1} u_{n}-B_{i} p\right)\right\|^{2} \\
& =\left\|x_{n}-p\right\|^{2}-\left\|\Lambda_{n}^{i-1} u_{n}-\Lambda_{n}^{k} u_{n}\right\|^{2}-\lambda_{i, n}^{2}\left\|B_{i} \Lambda_{n}^{i-1} u_{n}-B_{i} p\right\|^{2} \\
& \quad+2 \lambda_{i, n}\left\langle\Lambda_{n}^{i-1} u_{n}-\Lambda_{n}^{i} u_{n}, B_{i} \Lambda_{n}^{i-1} u_{n}-B_{i} p\right\rangle \\
& \leq\left\|x_{n}-p\right\|^{2}-\left\|\Lambda_{n}^{i-1} u_{n}-\Lambda_{n}^{i} u_{n}\right\|^{2}+2 \lambda_{i, n}\left\|\Lambda_{n}^{i-1} u_{n}-\Lambda_{n}^{i} u_{n}\right\|\left\|B_{i} \Lambda_{n}^{i-1} u_{n}-B_{i} p\right\| .
\end{aligned}
$$

Combining (3.20) and (3.23) we conclude that

$$
\begin{aligned}
\left\|y_{n}-p\right\|^{2} \leq & \beta_{n}\left\|x_{n}-p\right\|^{2}+\left(1-\beta_{n}\right)\left\|v_{n}-p\right\|^{2}+\alpha_{n} b\|p\|\left(2\left\|v_{n}-p\right\|+\alpha_{n} b\|p\|\right) \\
\leq & \beta_{n}\left\|x_{n}-p\right\|^{2}+\left(1-\beta_{n}\right)\left\|\Lambda_{n}^{i} u_{n}-p\right\|^{2}+\alpha_{n} b\|p\|\left(2\left\|v_{n}-p\right\|+\alpha_{n} b\|p\|\right) \\
\leq & \beta_{n}\left\|x_{n}-p\right\|^{2}+\left(1-\beta_{n}\right)\left[\left\|x_{n}-p\right\|^{2}-\left\|\Lambda_{n}^{i-1} u_{n}-\Lambda_{n}^{i} u_{n}\right\|^{2}\right. \\
& \left.+2 \lambda_{i, n}\left\|\Lambda_{n}^{i-1} u_{n}-\Lambda_{n}^{i} u_{n}\right\|\left\|B_{i} \Lambda_{n}^{i-1} u_{n}-B_{i} p\right\|\right] \\
& +\alpha_{n} b\|p\|\left(2\left\|v_{n}-p\right\|+\alpha_{n} b\|p\|\right) \\
\leq & \left\|x_{n}-p\right\|^{2}-\left(1-\beta_{n}\right)\left\|\Lambda_{n}^{i-1} u_{n}-\Lambda_{n}^{i} u_{n}\right\|^{2} \\
& +2 \lambda_{i, n}\left\|\Lambda_{n}^{i-1} u_{n}-\Lambda_{n}^{i} u_{n}\right\|\left\|B_{i} \Lambda_{n}^{i-1} u_{n}-B_{i} p\right\| \\
& +\alpha_{n} b\|p\|\left(2\left\|v_{n}-p\right\|+\alpha_{n} b\|p\|\right),
\end{aligned}
$$

which yields

$$
\begin{aligned}
(1- & \left.\beta_{n}\right)\left\|\Lambda_{n}^{i-1} u_{n}-\Lambda_{n}^{i} u_{n}\right\|^{2} \\
\leq & \left\|x_{n}-p\right\|^{2}-\left\|y_{n}-p\right\|^{2}+2 \lambda_{i, n}\left\|\Lambda_{n}^{i-1} u_{n}-\Lambda_{n}^{i} u_{n}\right\|\left\|B_{i} \Lambda_{n}^{i-1} u_{n}-B_{i} p\right\| \\
& +\alpha_{n} b\|p\|\left(2\left\|v_{n}-p\right\|+\alpha_{n} b\|p\|\right) \\
\leq & \left\|x_{n}-y_{n}\right\|\left(\left\|x_{n}-p\right\|+\left\|y_{n}-p\right\|\right)+2 \lambda_{i, n}\left\|\Lambda_{n}^{i-1} u_{n}-\Lambda_{n}^{i} u_{n}\right\|\left\|B_{i} \Lambda_{n}^{i-1} u_{n}-B_{i} p\right\| \\
& +\alpha_{n} b\|p\|\left(2\left\|v_{n}-p\right\|+\alpha_{n} b\|p\|\right) .
\end{aligned}
$$

Since $\left\{\beta_{n}\right\} \subset[c, d] \subset(0,1),\left\{\lambda_{i, n}\right\} \subset\left[a_{i}, b_{i}\right] \subset\left(0,2 \eta_{i}\right), i=1,2, \ldots, N$, and $\left\{u_{n}\right\},\left\{v_{n}\right\},\left\{x_{n}\right\}$ and $\left\{y_{n}\right\}$ are bounded sequences, we deduce from (3.17), (3.21), and $\alpha_{n} \rightarrow 0$ that

$$
\lim _{n \rightarrow \infty}\left\|\Lambda_{n}^{i-1} u_{n}-\Lambda_{n}^{i} u_{n}\right\|=0, \quad \forall i \in\{1,2, \ldots, N\}
$$


Also, combining (3.3), (3.20), and (3.22) we deduce that

$$
\begin{aligned}
\left\|y_{n}-p\right\|^{2} \leq & \beta_{n}\left\|x_{n}-p\right\|^{2}+\left(1-\beta_{n}\right)\left\|v_{n}-p\right\|^{2}+\alpha_{n} b\|p\|\left(2\left\|v_{n}-p\right\|+\alpha_{n} b\|p\|\right) \\
\leq & \beta_{n}\left\|x_{n}-p\right\|^{2}+\left(1-\beta_{n}\right)\left\|u_{n}-p\right\|^{2}+\alpha_{n} b\|p\|\left(2\left\|v_{n}-p\right\|+\alpha_{n} b\|p\|\right) \\
\leq & \beta_{n}\left\|x_{n}-p\right\|^{2}+\left(1-\beta_{n}\right)\left\|\Delta_{n}^{k} x_{n}-p\right\|^{2}+\alpha_{n} b\|p\|\left(2\left\|v_{n}-p\right\|+\alpha_{n} b\|p\|\right) \\
\leq & \beta_{n}\left\|x_{n}-p\right\|^{2}+\left(1-\beta_{n}\right)\left[\left\|x_{n}-p\right\|^{2}-\left\|\Delta_{n}^{k-1} x_{n}-\Delta_{n}^{k} x_{n}\right\|^{2}\right. \\
& \left.+2 r_{k, n}\left\|\Delta_{n}^{k-1} x_{n}-\Delta_{n}^{k} x_{n}\right\|\left\|A_{k} \Delta_{n}^{k-1} x_{n}-A_{k} p\right\|\right] \\
& +\alpha_{n} b\|p\|\left(2\left\|v_{n}-p\right\|+\alpha_{n} b\|p\|\right) \\
\leq & \left\|x_{n}-p\right\|^{2}-\left(1-\beta_{n}\right)\left\|\Delta_{n}^{k-1} x_{n}-\Delta_{n}^{k} x_{n}\right\|^{2} \\
& +2 r_{k, n}\left\|\Delta_{n}^{k-1} x_{n}-\Delta_{n}^{k} x_{n}\right\|\left\|A_{k} \Delta_{n}^{k-1} x_{n}-A_{k} p\right\| \\
& +\alpha_{n} b\|p\|\left(2\left\|v_{n}-p\right\|+\alpha_{n} b\|p\|\right),
\end{aligned}
$$

which yields

$$
\begin{aligned}
& \left(1-\beta_{n}\right)\left\|\Delta_{n}^{k-1} x_{n}-\Delta_{n}^{k} x_{n}\right\|^{2} \\
& \leq\left\|x_{n}-p\right\|^{2}-\left\|y_{n}-p\right\|^{2}+2 r_{k, n}\left\|\Delta_{n}^{k-1} x_{n}-\Delta_{n}^{k} x_{n}\right\|\left\|A_{k} \Delta_{n}^{k-1} x_{n}-A_{k} p\right\| \\
& \quad+\alpha_{n} b\|p\|\left(2\left\|v_{n}-p\right\|+\alpha_{n} b\|p\|\right) \\
& \leq\left\|x_{n}-y_{n}\right\|\left(\left\|x_{n}-p\right\|+\left\|y_{n}-p\right\|\right)+2 r_{k, n}\left\|\Delta_{n}^{k-1} x_{n}-\Delta_{n}^{k} x_{n}\right\|\left\|A_{k} \Delta_{n}^{k-1} x_{n}-A_{k} p\right\| \\
& \quad+\alpha_{n} b\|p\|\left(2\left\|v_{n}-p\right\|+\alpha_{n} b\|p\|\right) .
\end{aligned}
$$

Since $\left\{\beta_{n}\right\} \subset[c, d] \subset(0,1),\left\{r_{k, n}\right\} \subset\left[c_{k}, d_{k}\right] \subset\left(0,2 \mu_{k}\right)$ for $k=1,2, \ldots, M$, and $\left\{v_{n}\right\},\left\{x_{n}\right\},\left\{y_{n}\right\}$ are bounded sequences, we deduce from (3.17), (3.21), and $\alpha_{n} \rightarrow 0$ that

$$
\lim _{n \rightarrow \infty}\left\|\Delta_{n}^{k-1} x_{n}-\Delta_{n}^{k} x_{n}\right\|=0, \quad \forall k \in\{1,2, \ldots, M\} .
$$

Hence from (3.24) and (3.25) we get

$$
\begin{aligned}
\left\|x_{n}-u_{n}\right\| & =\left\|\Delta_{n}^{0} x_{n}-\Delta_{n}^{M} x_{n}\right\| \\
& \leq\left\|\Delta_{n}^{0} x_{n}-\Delta_{n}^{1} x_{n}\right\|+\left\|\Delta_{n}^{1} x_{n}-\Delta_{n}^{2} x_{n}\right\|+\cdots+\left\|\Delta_{n}^{M-1} x_{n}-\Delta_{n}^{M} x_{n}\right\| \\
& \rightarrow 0 \quad \text { as } n \rightarrow \infty
\end{aligned}
$$

and

$$
\begin{aligned}
\left\|u_{n}-v_{n}\right\| & =\left\|\Lambda_{n}^{0} u_{n}-\Lambda_{n}^{N} u_{n}\right\| \\
& \leq\left\|\Lambda_{n}^{0} u_{n}-\Lambda_{n}^{1} u_{n}\right\|+\left\|\Lambda_{n}^{1} u_{n}-\Lambda_{n}^{2} u_{n}\right\|+\cdots+\left\|\Lambda_{n}^{N-1} u_{n}-\Lambda_{n}^{N} u_{n}\right\| \\
& \rightarrow 0 \quad \text { as } n \rightarrow \infty,
\end{aligned}
$$

respectively. Thus, from (3.26) and (3.27) we obtain

$$
\begin{aligned}
\left\|x_{n}-v_{n}\right\| & \leq\left\|x_{n}-u_{n}\right\|+\left\|u_{n}-v_{n}\right\| \\
& \rightarrow 0 \quad \text { as } n \rightarrow \infty
\end{aligned}
$$


On the other hand, note that $\Gamma=\operatorname{VI}(C, \nabla f)$. Then, utilizing Lemma 2.1 and the $\frac{1}{L}$-inverse strong monotonicity of $\nabla f$, we deduce from (2.1) that

$$
\begin{aligned}
\left\|t_{n}-p\right\|^{2} \leq & \left\|\left(I-\lambda_{n} \nabla f_{\alpha_{n}}\right) v_{n}-\left(I-\lambda_{n} \nabla f\right) p\right\|^{2} \\
= & \left\|v_{n}-p-\lambda_{n}\left(\nabla f\left(v_{n}\right)-\nabla f(p)\right)-\lambda_{n} \alpha_{n} v_{n}\right\|^{2} \\
\leq & \left\|v_{n}-p-\lambda_{n}\left(\nabla f\left(v_{n}\right)-\nabla f(p)\right)\right\|^{2} \\
& \quad-2 \lambda_{n} \alpha_{n}\left\langle v_{n},\left(I-\lambda_{n} \nabla f_{\alpha_{n}}\right) v_{n}-\left(I-\lambda_{n} \nabla f\right) p\right\rangle \\
\leq & \left\|v_{n}-p\right\|^{2}+\lambda_{n}\left(\lambda_{n}-\frac{2}{L}\right)\left\|\nabla f\left(v_{n}\right)-\nabla f(p)\right\|^{2} \\
& +2 \alpha_{n} b\left\|v_{n}\right\|\left\|v_{n}-p-\lambda_{n}\left(\nabla f_{\alpha_{n}}\left(v_{n}\right)-\nabla f(p)\right)\right\| .
\end{aligned}
$$

Combining (3.4), (3.20), and (3.29) we obtain

$$
\begin{aligned}
\left\|y_{n}-p\right\|^{2} \leq & \beta_{n}\left\|x_{n}-p\right\|^{2}+\left(1-\beta_{n}\right)\left\|t_{n}-p\right\|^{2} \\
\leq & \beta_{n}\left\|x_{n}-p\right\|^{2}+\left(1-\beta_{n}\right)\left[\left\|v_{n}-p\right\|^{2}+\lambda_{n}\left(\lambda_{n}-\frac{2}{L}\right)\left\|\nabla f\left(v_{n}\right)-\nabla f(p)\right\|^{2}\right. \\
& \left.+2 \alpha_{n} b\left\|v_{n}\right\|\left\|v_{n}-p-\lambda_{n}\left(\nabla f_{\alpha_{n}}\left(v_{n}\right)-\nabla f(p)\right)\right\|\right] \\
\leq & \beta_{n}\left\|x_{n}-p\right\|^{2}+\left(1-\beta_{n}\right)\left[\left\|x_{n}-p\right\|^{2}+\lambda_{n}\left(\lambda_{n}-\frac{2}{L}\right)\left\|\nabla f\left(v_{n}\right)-\nabla f(p)\right\|^{2}\right. \\
& \left.+2 \alpha_{n} b\left\|v_{n}\right\|\left\|v_{n}-p-\lambda_{n}\left(\nabla f_{\alpha_{n}}\left(v_{n}\right)-\nabla f(p)\right)\right\|\right] \\
\leq & \left\|x_{n}-p\right\|^{2}+\left(1-\beta_{n}\right) \lambda_{n}\left(\lambda_{n}-\frac{2}{L}\right)\left\|\nabla f\left(v_{n}\right)-\nabla f(p)\right\|^{2} \\
& +2 \alpha_{n} b\left\|v_{n}\right\|\left\|v_{n}-p-\lambda_{n}\left(\nabla f_{\alpha_{n}}\left(v_{n}\right)-\nabla f(p)\right)\right\|
\end{aligned}
$$

which, together with $\left\{\lambda_{n}\right\} \subset[a, b] \subset\left(0, \frac{2}{L}\right)$ and $\left\{\beta_{n}\right\} \subset[c, d] \subset(0,1)$, leads to

$$
\begin{aligned}
(1 & -d) a\left(\frac{2}{L}-b\right)\left\|\nabla f\left(v_{n}\right)-\nabla f(p)\right\|^{2} \\
& \leq\left(1-\beta_{n}\right) \lambda_{n}\left(\frac{2}{L}-\lambda_{n}\right)\left\|\nabla f\left(v_{n}\right)-\nabla f(p)\right\|^{2} \\
& \leq\left\|x_{n}-p\right\|^{2}-\left\|y_{n}-p\right\|^{2}+2 \alpha_{n} b\left\|v_{n}\right\|\left\|v_{n}-p-\lambda_{n}\left(\nabla f_{\alpha_{n}}\left(v_{n}\right)-\nabla f(p)\right)\right\| \\
& \leq\left\|x_{n}-y_{n}\right\|\left(\left\|x_{n}-p\right\|+\left\|y_{n}-p\right\|\right)+2 \alpha_{n} b\left\|v_{n}\right\|\left\|v_{n}-p-\lambda_{n}\left(\nabla f_{\alpha_{n}}\left(v_{n}\right)-\nabla f(p)\right)\right\| .
\end{aligned}
$$

Since $\left\{v_{n}\right\},\left\{x_{n}\right\}$, and $\left\{y_{n}\right\}$ are bounded sequences, we deduce from (3.17) and $\alpha_{n} \rightarrow 0$ that

$$
\lim _{n \rightarrow \infty}\left\|\nabla f\left(v_{n}\right)-\nabla f(p)\right\|=0
$$

So, it is clear that

$$
\lim _{n \rightarrow \infty}\left\|\nabla f_{\alpha_{n}}\left(v_{n}\right)-\nabla f(p)\right\|=0
$$


Again, utilizing Proposition 2.1(iii), from $t_{n}=P_{C}\left(I-\lambda_{n} \nabla f_{\alpha_{n}}\right) v_{n}$ and $p=P_{C}\left(I-\lambda_{n} \nabla f\right) p$, we get

$$
\begin{aligned}
\| t_{n}- & p \|^{2} \\
= & \left\|P_{C}\left(I-\lambda_{n} \nabla f_{\alpha_{n}}\right) v_{n}-P_{C}\left(I-\lambda_{n} \nabla f\right) p\right\|^{2} \\
\leq & \left\langle\left(I-\lambda_{n} \nabla f_{\alpha_{n}}\right) v_{n}-\left(I-\lambda_{n} \nabla f\right) p, t_{n}-p\right\rangle \\
= & \frac{1}{2}\left(\left\|\left(I-\lambda_{n} \nabla f_{\alpha_{n}}\right) v_{n}-\left(I-\lambda_{n} \nabla f\right) p\right\|^{2}+\left\|t_{n}-p\right\|^{2}\right. \\
& \left.-\left\|\left(I-\lambda_{n} \nabla f_{\alpha_{n}}\right) v_{n}-\left(I-\lambda_{n} \nabla f\right) p-\left(t_{n}-p\right)\right\|^{2}\right) \\
= & \frac{1}{2}\left(\left\|\left(I-\lambda_{n} \nabla f_{\alpha_{n}}\right) v_{n}-\left(I-\lambda_{n} \nabla f_{\alpha_{n}}\right) p-\lambda_{n} \alpha_{n} p\right\|^{2}+\left\|t_{n}-p\right\|^{2}\right. \\
& \left.-\left\|\left(I-\lambda_{n} \nabla f_{\alpha_{n}}\right) v_{n}-\left(I-\lambda_{n} \nabla f\right) p-\left(t_{n}-p\right)\right\|^{2}\right) \\
= & \frac{1}{2}\left(\left\|\left(I-\lambda_{n} \nabla f_{\alpha_{n}}\right) v_{n}-\left(I-\lambda_{n} \nabla f_{\alpha_{n}}\right) p\right\|^{2}\right. \\
& -2 \lambda_{n} \alpha_{n}\left(p,\left(I-\lambda_{n} \nabla f_{\alpha_{n}}\right) v_{n}-\left(I-\lambda_{n} \nabla f\right) p\right\rangle \\
& \left.+\left\|t_{n}-p\right\|^{2}-\left\|\left(I-\lambda_{n} \nabla f_{\alpha_{n}}\right) v_{n}-\left(I-\lambda_{n} \nabla f\right) p-\left(t_{n}-p\right)\right\|^{2}\right) \\
\leq & \frac{1}{2}\left(\left\|v_{n}-p\right\|^{2}-2 \lambda_{n} \alpha_{n}\left(p,\left(I-\lambda_{n} \nabla f_{\alpha_{n}}\right) v_{n}-\left(I-\lambda_{n} \nabla f\right) p\right\rangle+\left\|t_{n}-p\right\|^{2}\right. \\
& \left.-\left\|\left(I-\lambda_{n} \nabla f_{\alpha_{n}}\right) v_{n}-\left(I-\lambda_{n} \nabla f\right) p-\left(t_{n}-p\right)\right\|^{2}\right) \\
\leq & \frac{1}{2}\left(\left\|v_{n}-p\right\|^{2}+2 \lambda_{n} \alpha_{n}\|p\|\left\|\left(I-\lambda_{n} \nabla f_{\alpha_{n}}\right) v_{n}-\left(I-\lambda_{n} \nabla f\right) p\right\|+\left\|t_{n}-p\right\|^{2}\right. \\
& \left.-\left\|v_{n}-t_{n}-\lambda_{n}\left(\nabla f_{\alpha_{n}}\left(v_{n}\right)-\nabla f(p)\right)\right\|^{2}\right),
\end{aligned}
$$

which immediately leads to

$$
\begin{aligned}
&\left\|t_{n}-p\right\|^{2} \leq\left\|v_{n}-p\right\|^{2}+2 \lambda_{n} \alpha_{n}\|p\|\left\|\left(I-\lambda_{n} \nabla f_{\alpha_{n}}\right) v_{n}-\left(I-\lambda_{n} \nabla f\right) p\right\| \\
&-\left\|v_{n}-t_{n}-\lambda_{n}\left(\nabla f_{\alpha_{n}}\left(v_{n}\right)-\nabla f(p)\right)\right\|^{2} .
\end{aligned}
$$

Combining (3.4), (3.20), and (3.31) we obtain

$$
\begin{aligned}
\left\|y_{n}-p\right\|^{2} \leq & \beta_{n}\left\|x_{n}-p\right\|^{2}+\left(1-\beta_{n}\right)\left\|t_{n}-p\right\|^{2} \\
\leq & \beta_{n}\left\|x_{n}-p\right\|^{2}+\left(1-\beta_{n}\right)\left[\left\|v_{n}-p\right\|^{2}\right. \\
& +2 \lambda_{n} \alpha_{n}\|p\|\left\|\left(I-\lambda_{n} \nabla f_{\alpha_{n}}\right) v_{n}-\left(I-\lambda_{n} \nabla f\right) p\right\| \\
& \left.-\left\|v_{n}-t_{n}-\lambda_{n}\left(\nabla f_{\alpha_{n}}\left(v_{n}\right)-\nabla f(p)\right)\right\|^{2}\right] \\
\leq & \beta_{n}\left\|x_{n}-p\right\|^{2}+\left(1-\beta_{n}\right)\left[\left\|x_{n}-p\right\|^{2}\right. \\
& +2 \lambda_{n} \alpha_{n}\|p\|\left\|\left(I-\lambda_{n} \nabla f_{\alpha_{n}}\right) v_{n}-\left(I-\lambda_{n} \nabla f\right) p\right\| \\
& \left.-\left\|v_{n}-t_{n}-\lambda_{n}\left(\nabla f_{\alpha_{n}}\left(v_{n}\right)-\nabla f(p)\right)\right\|^{2}\right] \\
\leq & \left\|x_{n}-p\right\|^{2}+2 \lambda_{n} \alpha_{n}\|p\|\left\|\left(I-\lambda_{n} \nabla f_{\alpha_{n}}\right) v_{n}-\left(I-\lambda_{n} \nabla f\right) p\right\| \\
& -\left(1-\beta_{n}\right)\left\|v_{n}-t_{n}-\lambda_{n}\left(\nabla f_{\alpha_{n}}\left(v_{n}\right)-\nabla f(p)\right)\right\|^{2},
\end{aligned}
$$


which immediately yields

$$
\begin{aligned}
& (1-d)\left\|v_{n}-t_{n}-\lambda_{n}\left(\nabla f_{\alpha_{n}}\left(v_{n}\right)-\nabla f(p)\right)\right\|^{2} \\
& \quad \leq\left(1-\beta_{n}\right)\left\|v_{n}-t_{n}-\lambda_{n}\left(\nabla f_{\alpha_{n}}\left(v_{n}\right)-\nabla f(p)\right)\right\|^{2} \\
& \quad \leq\left\|x_{n}-p\right\|^{2}-\left\|y_{n}-p\right\|^{2}+2 \lambda_{n} \alpha_{n}\|p\|\left\|\left(I-\lambda_{n} \nabla f_{\alpha_{n}}\right) v_{n}-\left(I-\lambda_{n} \nabla f\right) p\right\| \\
& \quad \leq\left\|x_{n}-y_{n}\right\|\left(\left\|x_{n}-p\right\|+\left\|y_{n}-p\right\|\right)+2 \alpha_{n} b\|p\|\left\|\left(I-\lambda_{n} \nabla f_{\alpha_{n}}\right) v_{n}-\left(I-\lambda_{n} \nabla f\right) p\right\| .
\end{aligned}
$$

Since $\left\{v_{n}\right\},\left\{x_{n}\right\}$, and $\left\{y_{n}\right\}$ are bounded sequences, we deduce from (3.17) and $\alpha_{n} \rightarrow 0$ that

$$
\lim _{n \rightarrow \infty}\left\|v_{n}-t_{n}-\lambda_{n}\left(\nabla f_{\alpha_{n}}\left(v_{n}\right)-\nabla f(p)\right)\right\|=0 .
$$

Observe that

$$
\left\|v_{n}-t_{n}\right\| \leq\left\|v_{n}-t_{n}-\lambda_{n}\left(\nabla f_{\alpha_{n}}\left(v_{n}\right)-\nabla f(p)\right)\right\|+\lambda_{n}\left\|\nabla f_{\alpha_{n}}\left(v_{n}\right)-\nabla f(p)\right\| .
$$

Thus, from (3.30) and (3.32) we have

$$
\lim _{n \rightarrow \infty}\left\|v_{n}-t_{n}\right\|=0
$$

Taking into account that $\left\|x_{n}-t_{n}\right\| \leq\left\|x_{n}-v_{n}\right\|+\left\|v_{n}-t_{n}\right\|$, from (3.28) and (3.33) we get

$$
\lim _{n \rightarrow \infty}\left\|x_{n}-t_{n}\right\|=0
$$

Utilizing the relation $y_{n}-x_{n}=\gamma_{n}\left(t_{n}-x_{n}\right)+\sigma_{n}\left(T t_{n}-x_{n}\right)$, we have

$$
\begin{aligned}
\left\|\sigma_{n}\left(T t_{n}-t_{n}\right)\right\| & =\left\|\sigma_{n}\left(T t_{n}-x_{n}\right)-\sigma_{n}\left(t_{n}-x_{n}\right)\right\| \\
& =\left\|y_{n}-x_{n}-\gamma_{n}\left(t_{n}-x_{n}\right)-\sigma_{n}\left(t_{n}-x_{n}\right)\right\| \\
& =\left\|y_{n}-x_{n}-\left(1-\beta_{n}\right)\left(t_{n}-x_{n}\right)\right\| \\
& \leq\left\|y_{n}-x_{n}\right\|+\left(1-\beta_{n}\right)\left\|t_{n}-x_{n}\right\| \\
& \leq\left\|y_{n}-x_{n}\right\|+\left\|t_{n}-x_{n}\right\|,
\end{aligned}
$$

which, together with (3.17) and (3.34), implies that

$$
\lim _{n \rightarrow \infty}\left\|\sigma_{n}\left(T t_{n}-t_{n}\right)\right\|=0
$$

Since $\liminf _{n \rightarrow \infty} \sigma_{n}>0$, we obtain

$$
\lim _{n \rightarrow \infty}\left\|t_{n}-T t_{n}\right\|=0
$$

Step 4. We prove that $\omega_{w}\left(x_{n}\right) \subset \Omega$.

Indeed, since $H$ is reflexive and $\left\{x_{n}\right\}$ is bounded, there exists at least a weak convergence subsequence of $\left\{x_{n}\right\}$. Hence we know that $\omega_{w}\left(x_{n}\right) \neq \emptyset$. Now, take an arbitrary $w \in \omega_{w}\left(x_{n}\right)$. Then there exists a subsequence $\left\{x_{n_{i}}\right\}$ of $\left\{x_{n}\right\}$ such that $x_{n_{i}} \rightarrow w$. From (3.24)-(3.26), (3.28), 
and (3.34) we have that $u_{n_{i}} \rightarrow w, v_{n_{i}} \rightarrow w, t_{n_{i}} \rightarrow w, \Lambda_{n_{i}}^{m} u_{n_{i}} \rightarrow w$ and $\Delta_{n_{i}}^{k} x_{n_{i}} \rightarrow w$, where $m \in\{1,2, \ldots, N\}$ and $k \in\{1,2, \ldots, M\}$. Utilizing Lemma 2.3(ii), we deduce from $t_{n_{i}} \rightarrow w$ and (3.35) that $w \in \operatorname{Fix}(T)$. Next, we prove that $w \in \bigcap_{m=1}^{N} \mathrm{I}\left(B_{m}, R_{m}\right)$. As a matter of fact, since $B_{m}$ is $\eta_{m}$-inverse-strongly monotone, $B_{m}$ is a monotone and Lipschitz-continuous mapping. It follows from Lemma 2.13 that $R_{m}+B_{m}$ is maximal monotone. Let $(v, g) \in$ $G\left(R_{m}+B_{m}\right)$, i.e., $g-B_{m} v \in R_{m} v$. Again, since $\Lambda_{n}^{m} u_{n}=J_{R_{m}, \lambda_{m, n}}\left(I-\lambda_{m, n} B_{m}\right) \Lambda_{n}^{m-1} u_{n}, n \geq 1$, $m \in\{1,2, \ldots, N\}$, we have

$$
\Lambda_{n}^{m-1} u_{n}-\lambda_{m, n} B_{m} \Lambda_{n}^{m-1} u_{n} \in\left(I+\lambda_{m, n} R_{m}\right) \Lambda_{n}^{m} u_{n}
$$

that is,

$$
\frac{1}{\lambda_{m, n}}\left(\Lambda_{n}^{m-1} u_{n}-\Lambda_{n}^{m} u_{n}-\lambda_{m, n} B_{m} \Lambda_{n}^{m-1} u_{n}\right) \in R_{m} \Lambda_{n}^{m} u_{n} .
$$

In terms of the monotonicity of $R_{m}$, we get

$$
\left\langle v-\Lambda_{n}^{m} u_{n}, g-B_{m} v-\frac{1}{\lambda_{m, n}}\left(\Lambda_{n}^{m-1} u_{n}-\Lambda_{n}^{m} u_{n}-\lambda_{m, n} B_{m} \Lambda_{n}^{m-1} u_{n}\right)\right\rangle \geq 0
$$

and hence

$$
\begin{aligned}
\langle v & \left.-\Lambda_{n}^{m} u_{n}, g\right\rangle \\
& \geq\left\langle v-\Lambda_{n}^{m} u_{n}, B_{m} v+\frac{1}{\lambda_{m, n}}\left(\Lambda_{n}^{m-1} u_{n}-\Lambda_{n}^{m} u_{n}-\lambda_{m, n} B_{m} \Lambda_{n}^{m-1} u_{n}\right)\right\rangle \\
& =\left\langle v-\Lambda_{n}^{m} u_{n}, B_{m} v-B_{m} \Lambda_{n}^{m} u_{n}+B_{m} \Lambda_{n}^{m} u_{n}-B_{m} \Lambda_{n}^{m-1} u_{n}+\frac{1}{\lambda_{m, n}}\left(\Lambda_{n}^{m-1} u_{n}-\Lambda_{n}^{m} u_{n}\right)\right\rangle \\
& \geq\left\langle v-\Lambda_{n}^{m} u_{n}, B_{m} \Lambda_{n}^{m} u_{n}-B_{m} \Lambda_{n}^{m-1} u_{n}\right\rangle+\left\langle v-\Lambda_{n}^{m} u_{n}, \frac{1}{\lambda_{m, n}}\left(\Lambda_{n}^{m-1} u_{n}-\Lambda_{n}^{m} u_{n}\right)\right\rangle .
\end{aligned}
$$

In particular,

$$
\begin{aligned}
\left\langle v-\Lambda_{n_{i}}^{m} u_{n_{i}}, g\right\rangle \geq & \left\langle v-\Lambda_{n_{i}}^{m} u_{n_{i}}, B_{m} \Lambda_{n_{i}}^{m} u_{n_{i}}-B_{m} \Lambda_{n_{i}}^{m-1} u_{n_{i}}\right\rangle \\
& +\left\langle v-\Lambda_{n_{i}}^{m} u_{n_{i}}, \frac{1}{\lambda_{m, n_{i}}}\left(\Lambda_{n_{i}}^{m-1} u_{n_{i}}-\Lambda_{n_{i}}^{m} u_{n_{i}}\right)\right\rangle .
\end{aligned}
$$

Since $\left\|\Lambda_{n}^{m} u_{n}-\Lambda_{n}^{m-1} u_{n}\right\| \rightarrow 0$ (due to (3.24)) and $\left\|B_{m} \Lambda_{n}^{m} u_{n}-B_{m} \Lambda_{n}^{m-1} u_{n}\right\| \rightarrow 0$ (due to the Lipschitz-continuity of $\left.B_{m}\right)$, we conclude from $\Lambda_{n_{i}}^{m} u_{n_{i}} \rightarrow w$ and $\left\{\lambda_{i, n}\right\} \subset\left[a_{i}, b_{i}\right] \subset\left(0,2 \eta_{i}\right)$ that

$$
\lim _{i \rightarrow \infty}\left\langle v-\Lambda_{n_{i}}^{m} u_{n_{i}}, g\right\rangle=\langle v-w, g\rangle \geq 0 .
$$

It follows from the maximal monotonicity of $B_{m}+R_{m}$ that $0 \in\left(R_{m}+B_{m}\right) w$, i.e., $w \in$ $\mathrm{I}\left(B_{m}, R_{m}\right)$. Therefore, $w \in \bigcap_{m=1}^{N} \mathrm{I}\left(B_{m}, R_{m}\right)$. Next we prove that $w \in \bigcap_{k=1}^{M} \operatorname{GMEP}\left(\Theta_{k}, \varphi_{k}, A_{k}\right)$. Since $\Delta_{n}^{k} x_{n}=T_{r_{k, n}}^{\left(\Theta_{k}, \varphi_{k}\right)}\left(I-r_{k, n} A_{k}\right) \Delta_{n}^{k-1} x_{n}, n \geq 1, k \in\{1,2, \ldots, M\}$, we have

$$
\begin{aligned}
& \Theta_{k}\left(\Delta_{n}^{k} x_{n}, y\right)+\varphi_{k}(y)-\varphi_{k}\left(\Delta_{n}^{k} x_{n}\right)+\left\langle A_{k} \Delta_{n}^{k-1} x_{n}, y-\Delta_{n}^{k} x_{n}\right\rangle \\
& +\frac{1}{r_{k, n}}\left\langle y-\Delta_{n}^{k} x_{n}, \Delta_{n}^{k} x_{n}-\Delta_{n}^{k-1} x_{n}\right\rangle \geq 0 .
\end{aligned}
$$


By (A2), we have

$$
\begin{aligned}
& \varphi_{k}(y)-\varphi_{k}\left(\Delta_{n}^{k} x_{n}\right)+\left\langle A_{k} \Delta_{n}^{k-1} x_{n}, y-\Delta_{n}^{k} x_{n}\right\rangle+\frac{1}{r_{k, n}}\left\langle y-\Delta_{n}^{k} x_{n}, \Delta_{n}^{k} x_{n}-\Delta_{n}^{k-1} x_{n}\right\rangle \\
& \geq \Theta_{k}\left(y, \Delta_{n}^{k} x_{n}\right) .
\end{aligned}
$$

Let $z_{t}=t y+(1-t) w$ for all $t \in(0,1]$ and $y \in C$. This implies that $z_{t} \in C$. Then we have

$$
\begin{aligned}
\left\langle z_{t}-\right. & \left.\Delta_{n}^{k} x_{n}, A_{k} z_{t}\right\rangle \\
\geq & \varphi_{k}\left(\Delta_{n}^{k} x_{n}\right)-\varphi_{k}\left(z_{t}\right)+\left\langle z_{t}-\Delta_{n}^{k} x_{n}, A_{k} z_{t}\right\rangle-\left\langle z_{t}-\Delta_{n}^{k} x_{n}, A_{k} \Delta_{n}^{k-1} x_{n}\right\rangle \\
& \quad-\left\langle z_{t}-\Delta_{n}^{k} x_{n}, \frac{\Delta_{n}^{k} x_{n}-\Delta_{n}^{k-1} x_{n}}{r_{k, n}}\right\rangle+\Theta_{k}\left(z_{t}, \Delta_{n}^{k} x_{n}\right) \\
= & \varphi_{k}\left(\Delta_{n}^{k} x_{n}\right)-\varphi_{k}\left(z_{t}\right)+\left\langle z_{t}-\Delta_{n}^{k} x_{n}, A_{k} z_{t}-A_{k} \Delta_{n}^{k} x_{n}\right\rangle \\
& +\left\langle z_{t}-\Delta_{n}^{k} x_{n}, A_{k} \Delta_{n}^{k} x_{n}-A_{k} \Delta_{n}^{k-1} x_{n}\right\rangle \\
& \quad-\left\langle z_{t}-\Delta_{n}^{k} x_{n}, \frac{\Delta_{n}^{k} x_{n}-\Delta_{n}^{k-1} x_{n}}{r_{k, n}}\right\rangle+\Theta_{k}\left(z_{t}, \Delta_{n}^{k} x_{n}\right) .
\end{aligned}
$$

By (3.25), we have $\left\|A_{k} \Delta_{n}^{k} x_{n}-A_{k} \Delta_{n}^{k-1} x_{n}\right\| \rightarrow 0$ as $n \rightarrow \infty$. Furthermore, by the monotonicity of $A_{k}$, we obtain $\left\langle z_{t}-\Delta_{n}^{k} x_{n}, A_{k} z_{t}-A_{k} \Delta_{n}^{k} x_{n}\right\rangle \geq 0$. Then by (A4) we obtain

$$
\left\langle z_{t}-w, A_{k} z_{t}\right\rangle \geq \varphi_{k}(w)-\varphi_{k}\left(z_{t}\right)+\Theta_{k}\left(z_{t}, w\right) .
$$

Utilizing (A1), (A4), and (3.37), we obtain

$$
\begin{aligned}
0 & =\Theta_{k}\left(z_{t}, z_{t}\right)+\varphi_{k}\left(z_{t}\right)-\varphi_{k}\left(z_{t}\right) \\
& \leq t \Theta_{k}\left(z_{t}, y\right)+(1-t) \Theta_{k}\left(z_{t}, w\right)+t \varphi_{k}(y)+(1-t) \varphi_{k}(w)-\varphi_{k}\left(z_{t}\right) \\
& \leq t\left[\Theta_{k}\left(z_{t}, y\right)+\varphi_{k}(y)-\varphi_{k}\left(z_{t}\right)\right]+(1-t)\left\langle z_{t}-w, A_{k} z_{t}\right\rangle \\
& =t\left[\Theta_{k}\left(z_{t}, y\right)+\varphi_{k}(y)-\varphi_{k}\left(z_{t}\right)\right]+(1-t) t\left\langle y-w, A_{k} z_{t}\right\rangle
\end{aligned}
$$

and hence

$$
0 \leq \Theta_{k}\left(z_{t}, y\right)+\varphi_{k}(y)-\varphi_{k}\left(z_{t}\right)+(1-t)\left\langle y-w, A_{k} z_{t}\right\rangle
$$

Letting $t \rightarrow 0$, we have, for each $y \in C$,

$$
0 \leq \Theta_{k}(w, y)+\varphi_{k}(y)-\varphi_{k}(w)+\left\langle y-w, A_{k} w\right\rangle .
$$

This implies that $w \in \operatorname{GMEP}\left(\Theta_{k}, \varphi_{k}, A_{k}\right)$ and hence $w \in \bigcap_{k=1}^{M} \operatorname{GMEP}\left(\Theta_{k}, \varphi_{k}, A_{k}\right)$. Thus, $w \in$ $\Omega=\bigcap_{n=1}^{\infty} \operatorname{Fix}\left(T_{n}\right) \cap \bigcap_{k=1}^{M} \operatorname{GMEP}\left(\Theta_{k}, \varphi_{k}, A_{k}\right) \cap \bigcap_{m=1}^{N} \mathrm{I}\left(B_{m}, R_{m}\right)$.

Furthermore, let us show that $w \in \Gamma$. In fact, define

$$
\widetilde{T} v= \begin{cases}\nabla f(v)+N_{C} v, & \text { if } v \in C, \\ \emptyset, & \text { if } v \notin C,\end{cases}
$$


where $N_{C} v=\{u \in H:\langle v-x, u\rangle \geq 0, \forall x \in C\}$. Then $\widetilde{T}$ is maximal monotone and $0 \in \widetilde{T} v$ if and only if $v \in \operatorname{VI}(C, \nabla f)$; see [29]. Let $(v, \tilde{v}) \in G(\widetilde{T})$. Then we have $\tilde{v} \in \widetilde{T} v=\nabla f(v)+N_{C} v$, and hence $\tilde{v}-\nabla f(v) \in N_{C} v$. So, we have $\langle v-x, \tilde{v}-\nabla f(v)\rangle \geq 0$ for all $x \in C$. On the other hand, from $t_{n}=P_{C}\left(v_{n}-\lambda_{n} \nabla f_{\alpha_{n}}\left(v_{n}\right)\right)$ and $v \in C$, we get $\left\langle v_{n}-\lambda_{n} \nabla f_{\alpha_{n}}\left(v_{n}\right)-t_{n}, t_{n}-v\right\rangle \geq 0$, and hence,

$$
\left\langle v-t_{n}, \frac{t_{n}-v_{n}}{\lambda_{n}}+\nabla f_{\alpha_{n}}\left(v_{n}\right)\right\rangle \geq 0 .
$$

Therefore, from $\tilde{v}-\nabla f(v) \in N_{C} v$ and $t_{n_{i}} \in C$, we have

$$
\begin{aligned}
\left\langle v-t_{n_{i}}, \tilde{v}\right\rangle \geq & \left\langle v-t_{n_{i}}, \nabla f(v)\right\rangle \\
\geq & \left\langle v-t_{n_{i}}, \nabla f(v)\right\rangle-\left\langle v-t_{n_{i}}, \frac{t_{n_{i}}-v_{n_{i}}}{\lambda_{n_{i}}}+\nabla f_{\alpha_{n_{i}}}\left(v_{n_{i}}\right)\right\rangle \\
= & \left\langle v-t_{n_{i}}, \nabla f(v)\right\rangle-\left\langle v-t_{n_{i}}, \frac{t_{n_{i}}-v_{n_{i}}}{\lambda_{n_{i}}}+\nabla f\left(v_{n_{i}}\right)\right\rangle-\alpha_{n_{i}}\left\langle v-t_{n_{i}}, v_{n_{i}}\right\rangle \\
= & \left\langle v-t_{n_{i}}, \nabla f(v)-\nabla f\left(t_{n_{i}}\right)\right\rangle+\left\langle v-t_{n_{i}}, \nabla f\left(t_{n_{i}}\right)-\nabla f\left(v_{n_{i}}\right)\right\rangle \\
& -\left\langle v-t_{n_{i}}, \frac{t_{n_{i}}-v_{n_{i}}}{\lambda_{n_{i}}}\right\rangle-\alpha_{n_{i}}\left\langle v-t_{n_{i}}, v_{n_{i}}\right\rangle \\
\geq & \left\langle v-t_{n_{i}}, \nabla f\left(t_{n_{i}}\right)-\nabla f\left(v_{n_{i}}\right)\right\rangle-\left\langle v-t_{n_{i}}, \frac{t_{n_{i}}-v_{n_{i}}}{\lambda_{n_{i}}}\right\rangle-\alpha_{n_{i}}\left\langle v-t_{n_{i}}, v_{n_{i}}\right\rangle .
\end{aligned}
$$

Hence, it is easy to see that $\langle v-w, \tilde{v}\rangle \geq 0$ as $i \rightarrow \infty$. Since $\widetilde{T}$ is maximal monotone, we have $w \in \widetilde{T}^{-1} 0$, and hence $w \in \operatorname{VI}(C, \nabla f)=\Gamma$. Consequently, $w \in \bigcap_{k=1}^{M} \operatorname{GMEP}\left(\Theta_{k}, \varphi_{k}, A_{k}\right) \cap$ $\bigcap_{i=1}^{N} \mathrm{I}\left(B_{i}, R_{i}\right) \cap \operatorname{Fix}(T) \cap \Gamma=: \Omega$. This shows that $\omega_{w}\left(x_{n}\right) \subset \Omega$.

Step 5. We prove that $x_{n} \rightarrow x^{*}$ where $\left\{x^{*}\right\}=\operatorname{VI}(\Omega, \gamma S-\mu F)$.

Indeed, take an arbitrary $w \in \omega_{w}\left(x_{n}\right)$. Then there exists a subsequence $\left\{x_{n_{i}}\right\}$ of $\left\{x_{n}\right\}$ such that $x_{n_{i}} \rightarrow w$. Utilizing (3.16), we find that, for all $p \in \Omega$,

$$
\begin{aligned}
&\left\|x_{n+1}-p\right\|^{2} \\
& \leq\left(1-\epsilon_{n} \frac{\tau^{2}-\gamma^{2}}{\tau}\right)\left(\left\|x_{n}-p\right\|+\alpha_{n} b\|p\|\right)^{2}-\frac{\beta_{n}\left(1-\epsilon_{n} \tau\right)}{1-\beta_{n}}\left\|y_{n}-x_{n}\right\|^{2} \\
&+2 \epsilon_{n} \delta_{n}\left((\gamma V p-\mu F p), x_{n+1}-p\right\rangle+2 \epsilon_{n}\left(1-\delta_{n}\right)\left\langle(\gamma S p-\mu F p), x_{n+1}-p\right\rangle \\
& \leq\left(\left\|x_{n}-p\right\|+\alpha_{n} b\|p\|\right)^{2}+2 \epsilon_{n} \delta_{n}\left\langle(\gamma V p-\mu F p), x_{n+1}-p\right\rangle \\
&+2 \epsilon_{n}\left(1-\delta_{n}\right)\left\langle(\gamma S p-\mu F p), x_{n+1}-p\right\rangle,
\end{aligned}
$$

which implies that

$$
\begin{aligned}
& \left\langle(\mu F-\gamma S) p, x_{n}-p\right\rangle \\
& \leq\left\langle(\mu F-\gamma S) p, x_{n}-x_{n+1}\right\rangle+\left\langle(\mu F-\gamma S) p, x_{n+1}-p\right\rangle \\
& \leq\|(\mu F-\gamma S) p\|\left\|x_{n}-x_{n+1}\right\|+\frac{\left(\left\|x_{n}-p\right\|+\alpha_{n} b\|p\|\right)^{2}-\left\|x_{n+1}-p\right\|^{2}}{2 \epsilon_{n}\left(1-\delta_{n}\right)} \\
& \quad+\frac{\delta_{n}}{1-\delta_{n}}\left\langle(\gamma V-\mu F) p, x_{n+1}-p\right\rangle
\end{aligned}
$$




$$
\begin{aligned}
\leq & \|(\mu F-\gamma S) p\|\left\|x_{n}-x_{n+1}\right\| \\
& +\frac{\left(\left\|x_{n}-x_{n+1}\right\|+\alpha_{n} b\|p\|\right)\left(\left\|x_{n}-p\right\|+\left\|x_{n+1}-p\right\|+\alpha_{n} b\|p\|\right)}{2 \epsilon_{n}\left(1-\delta_{n}\right)} \\
& +\frac{\delta_{n}}{1-\delta_{n}}\|(\gamma V-\mu F) p\|\left\|x_{n+1}-p\right\| .
\end{aligned}
$$

Since $\alpha_{n} \rightarrow 0, \frac{\alpha_{n}}{\delta_{n}} \rightarrow 0,\left\|x_{n}-x_{n+1}\right\| \rightarrow 0$, and

$$
\lim _{n \rightarrow \infty} \frac{\left\|x_{n}-x_{n+1}\right\|+\alpha_{n} b\|p\|}{\epsilon_{n}}=\lim _{n \rightarrow \infty} \frac{\left\|x_{n}-x_{n+1}\right\|+\alpha_{n} b\|p\|}{\delta_{n}} \cdot \frac{\delta_{n}}{\epsilon_{n}}=0
$$

from (3.38) we conclude that

$$
\begin{aligned}
\langle(\mu F-\gamma S) p, w-p\rangle & =\lim _{i \rightarrow \infty}\left\langle(\mu F-\gamma S) p, x_{n_{i}}-p\right\rangle \\
& \leq \limsup _{n \rightarrow \infty}\left\langle(\mu F-\gamma S) p, x_{n}-p\right\rangle \\
& \leq 0, \quad \forall p \in \Omega,
\end{aligned}
$$

that is,

$$
\langle(\mu F-\gamma S) p, w-p\rangle \leq 0, \quad \forall p \in \Omega
$$

Since $\mu F-\gamma S$ is $(\mu \eta-\gamma)$-strongly monotone and $(\mu \kappa+\gamma)$-Lipschitz-continuous, by Minty's lemma [39] we know that (3.39) is equivalent to the VIP

$$
\langle(\mu F-\gamma S) w, p-w\rangle \geq 0, \quad \forall p \in \Omega .
$$

This shows that $w \in \operatorname{VI}(\Omega, \mu F-\gamma S)$. Taking into account $\left\{x^{*}\right\}=\operatorname{VI}(\Omega, \mu F-\gamma S)$, we know that $w=x^{*}$. Thus, $\omega_{w}\left(x_{n}\right)=\left\{x^{*}\right\}$; that is, $x_{n} \rightarrow x^{*}$.

Next we prove that $\lim _{n \rightarrow \infty}\left\|x_{n}-x^{*}\right\|=0$. As a matter of fact, utilizing (3.16) with $p=x^{*}$, we get

$$
\begin{aligned}
&\left\|x_{n+1}-x^{*}\right\|^{2} \\
& \leq\left(1-\epsilon_{n} \frac{\tau^{2}-\gamma^{2}}{\tau}\right)\left(\left\|x_{n}-x^{*}\right\|+\alpha_{n} b\left\|x^{*}\right\|\right)^{2}-\frac{\beta_{n}\left(1-\epsilon_{n} \tau\right)}{1-\beta_{n}}\left\|y_{n}-x_{n}\right\|^{2} \\
&+2 \epsilon_{n} \delta_{n}\left(\left(\gamma V x^{*}-\mu F x^{*}\right), x_{n+1}-x^{*}\right\rangle+2 \epsilon_{n}\left(1-\delta_{n}\right)\left\langle\left(\gamma S x^{*}-\mu F x^{*}\right), x_{n+1}-x^{*}\right\rangle \\
& \leq\left(1-\epsilon_{n} \frac{\tau^{2}-\gamma^{2}}{\tau}\right)\left(\left\|x_{n}-x^{*}\right\|+\alpha_{n} b\left\|x^{*}\right\|\right)^{2}+2 \epsilon_{n} \delta_{n}\left\|(\gamma V-\mu F) x^{*}\right\|\left\|x_{n+1}-x^{*}\right\| \\
&\left.+2 \epsilon_{n}\left(1-\delta_{n}\right)(\gamma S-\mu F) x^{*}, x_{n+1}-x^{*}\right\rangle \\
&=\left(1-\epsilon_{n} \frac{\tau^{2}-\gamma^{2}}{\tau}\right)\left[\left\|x_{n}-x^{*}\right\|^{2}+\alpha_{n} b\left\|x^{*}\right\|\left(2\left\|x_{n}-x^{*}\right\|+\alpha_{n} b\left\|x^{*}\right\|\right)\right] \\
&+2 \epsilon_{n} \delta_{n}\left\|(\gamma V-\mu F) x^{*}\right\|\left\|x_{n+1}-x^{*}\right\|+2 \epsilon_{n}\left(1-\delta_{n}\right)\left\langle(\gamma S-\mu F) x^{*}, x_{n+1}-x^{*}\right\rangle \\
& \leq\left(1-\epsilon_{n} \frac{\tau^{2}-\gamma^{2}}{\tau}\right)\left\|x_{n}-x^{*}\right\|^{2}+2 \epsilon_{n} \delta_{n}\left\|(\gamma V-\mu F) x^{*}\right\|\left\|x_{n+1}-x^{*}\right\|
\end{aligned}
$$




$$
\begin{aligned}
& +2 \epsilon_{n}\left(1-\delta_{n}\right)\left\langle(\gamma S-\mu F) x^{*}, x_{n+1}-x^{*}\right\rangle+\alpha_{n} b\left\|x^{*}\right\|\left(2\left\|x_{n}-x^{*}\right\|+\alpha_{n} b\left\|x^{*}\right\|\right) \\
= & \left(1-\epsilon_{n} \frac{\tau^{2}-\gamma^{2}}{\tau}\right)\left\|x_{n}-x^{*}\right\|^{2}+\epsilon_{n} \frac{\tau^{2}-\gamma^{2}}{\tau} \cdot \frac{2 \tau}{\tau^{2}-\gamma^{2}}\left[\delta_{n}\left\|(\gamma V-\mu F) x^{*}\right\|\left\|x_{n+1}-x^{*}\right\|\right. \\
& \left.+\left(1-\delta_{n}\right)\left\langle(\gamma S-\mu F) x^{*}, x_{n+1}-x^{*}\right\rangle\right]+\alpha_{n} b\left\|x^{*}\right\|\left(2\left\|x_{n}-x^{*}\right\|+\alpha_{n} b\left\|x^{*}\right\|\right) .
\end{aligned}
$$

Since $\sum_{n=0}^{\infty} \alpha_{n}<\infty, \sum_{n=0}^{\infty} \epsilon_{n}=\infty$, and $\lim _{n \rightarrow \infty}\left\langle(\gamma S-\mu F) x^{*}, x^{*}-x_{n+1}\right\rangle=0$ (due to $x_{n} \rightarrow x^{*}$ ), we deduce that $\sum_{n=0}^{\infty} \alpha_{n} b\left\|x^{*}\right\|\left(2\left\|x_{n}-x^{*}\right\|+\alpha_{n} b\left\|x^{*}\right\|\right)<\infty, \sum_{n=0}^{\infty} \epsilon_{n} \frac{\tau^{2}-\gamma^{2}}{\tau}=\infty$, and

$$
\lim _{n \rightarrow \infty} \frac{2 \tau}{\tau^{2}-\gamma^{2}}\left[\delta_{n}\left\|(\gamma V-\mu F) x^{*}\right\|\left\|x_{n+1}-x^{*}\right\|+\left(1-\delta_{n}\right)\left\langle(\gamma S-\mu F) x^{*}, x_{n+1}-x^{*}\right\rangle\right]=0
$$

Therefore, applying Lemma 2.8 to (3.41) we infer that $\lim _{n \rightarrow \infty}\left\|x_{n}-x^{*}\right\|=0$. This completes the proof.

In Theorem 3.1, putting $M=1$ and $N=2$, we obtain the following.

Corollary 3.1 Let $C$ be a nonempty closed convex subset of a real Hilbert space $H$. Let $f$ : $C \rightarrow \mathbf{R}$ be a convex functional with L-Lipschitz-continuous gradient $\nabla f$. Let $\Theta_{1}$ be a bifunction from $C \times C$ to $\mathbf{R}$ satisfying (A1)-(A4), $\varphi_{1}: C \rightarrow \mathbf{R} \cup\{+\infty\}$ be a proper lower semicontinuous and convex function with restriction (B1) or (B2), and $A_{1}: H \rightarrow H$ be $\mu_{1}$-inversestrongly monotone. Let $R_{i}: C \rightarrow 2^{H}$ be a maximal monotone mapping and $B_{i}: C \rightarrow H$ be $\eta_{i}$-inverse-strongly monotone, for $i=1,2$. Let $T: C \rightarrow C$ be a $\xi$-strictly pseudocontractive mapping, $S: H \rightarrow H$ be a nonexpansive mapping and $V: H \rightarrow H$ be a $\rho$-contraction with coefficient $\rho \in[0,1)$. Let $F: H \rightarrow H$ be $\kappa$-Lipschitzian and $\eta$-strongly monotone with positive constants $\kappa, \eta>0$ such that $0 \leq \gamma<\tau$ and $0<\mu<\frac{2 \eta}{\kappa^{2}}$ where $\tau=1-\sqrt{1-\mu\left(2 \eta-\mu \kappa^{2}\right)}$. Assume that $\Omega:=\operatorname{GMEP}\left(\Theta_{1}, \varphi_{1}, A_{1}\right) \cap \mathrm{I}\left(B_{2}, R_{2}\right) \cap \mathrm{I}\left(B_{1}, R_{1}\right) \cap \operatorname{Fix}(T) \cap \Gamma \neq \emptyset$. Let $\left\{\lambda_{n}\right\} \subset$ $[a, b] \subset\left(0, \frac{2}{L}\right),\left\{\alpha_{n}\right\} \subset(0, \infty)$ with $\sum_{n=0}^{\infty} \alpha_{n}<\infty,\left\{\epsilon_{n}\right\},\left\{\delta_{n}\right\},\left\{\beta_{n}\right\},\left\{\gamma_{n}\right\},\left\{\sigma_{n}\right\} \subset(0,1)$ with $\beta_{n}+\gamma_{n}+\sigma_{n}=1$, and $\left\{r_{1, n}\right\} \subset\left[c_{1}, d_{1}\right] \subset\left(0,2 \mu_{1}\right),\left\{\lambda_{i, n}\right\} \subset\left[a_{i}, b_{i}\right] \subset\left(0,2 \eta_{i}\right)$ for $i=1,2$. For arbitrarily given $x_{0} \in H$, let $\left\{x_{n}\right\}$ be a sequence generated by

$$
\left\{\begin{array}{l}
\Theta\left(u_{n}, y\right)+\varphi(y)-\varphi\left(u_{n}\right)+\left\langle A x_{n}, y-u_{n}\right\rangle+\frac{1}{r_{n}}\left\langle y-u_{n}, u_{n}-x_{n}\right\rangle \geq 0, \quad \forall y \in C, \\
v_{n}=J_{R_{2}, \lambda_{2, n}}\left(I-\lambda_{2, n} B_{2}\right) J_{R_{1}, \lambda_{1, n}}\left(I-\lambda_{1, n} B_{1}\right) u_{n}, \\
y_{n}=\beta_{n} x_{n}+\gamma_{n} P_{C}\left(I-\lambda_{n} \nabla f_{\alpha_{n}}\right) v_{n}+\sigma_{n} T P_{C}\left(I-\lambda_{n} \nabla f_{\alpha_{n}}\right) v_{n} \\
x_{n+1}=\epsilon_{n} \gamma\left(\delta_{n} V x_{n}+\left(1-\delta_{n}\right) S x_{n}\right)+\left(I-\epsilon_{n} \mu F\right) y_{n}, \quad \forall n \geq 0
\end{array}\right.
$$

where $\nabla f_{\alpha_{n}}=\alpha_{n} I+\nabla f$ for all $n \geq 0$. Suppose that

(C1) $\lim _{n \rightarrow \infty} \epsilon_{n}=0, \sum_{n=0}^{\infty} \epsilon_{n}=\infty$ and $\lim _{n \rightarrow \infty} \frac{1}{\epsilon_{n}}\left|1-\frac{\delta_{n-1}}{\delta_{n}}\right|=0$;

(C2) $\lim \sup _{n \rightarrow \infty} \frac{\delta_{n}}{\epsilon_{n}}<\infty, \lim _{n \rightarrow \infty} \frac{1}{\epsilon_{n}}\left|\frac{1}{\delta_{n}}-\frac{1}{\delta_{n-1}}\right|=0$ and $\lim _{n \rightarrow \infty} \frac{1}{\delta_{n}}\left|1-\frac{\epsilon_{n-1}}{\epsilon_{n}}\right|=0$;

(C3) $\lim _{n \rightarrow \infty} \frac{\left|\beta_{n}-\beta_{n-1}\right|}{\epsilon_{n} \delta_{n}}=0$ and $\lim _{n \rightarrow \infty} \frac{\left|\gamma_{n}-\gamma_{n-1}\right|}{\epsilon_{n} \delta_{n}}=0$;

(C4) $\lim _{n \rightarrow \infty} \frac{\left|r_{1, n}-r_{1, n-1}\right|}{\epsilon_{n} \delta_{n}}=0$ and $\lim _{n \rightarrow \infty} \frac{\left|\lambda_{i, n}-\lambda_{i, n-1}\right|}{\epsilon_{n} \delta_{n}}=0$ for $i=1,2$;

(C5) $\lim _{n \rightarrow \infty} \frac{\left|\lambda_{n}-\lambda_{n-1}\right|}{\epsilon_{n} \delta_{n}}=0, \lim _{n \rightarrow \infty} \frac{\left|\lambda_{n} \alpha_{n}-\lambda_{n-1} \alpha_{n-1}\right|}{\epsilon_{n} \delta_{n}}=0$ and $\left(\gamma_{n}+\sigma_{n}\right) \xi \leq \gamma_{n}$ for all $n \geq 0$;

(C6) $\left\{\beta_{n}\right\} \subset[c, d] \subset(0,1), \lim _{n \rightarrow \infty} \frac{\alpha_{n}}{\delta_{n}}=0$ and $\liminf _{n \rightarrow \infty} \sigma_{n}>0$.

Then we have:

(i) $\lim _{n \rightarrow \infty} \frac{\left\|x_{n+1}-x_{n}\right\|}{\delta_{n}}=0$;

(ii) $\omega_{w}\left(x_{n}\right) \subset \Omega$; 
(iii) $\left\{x_{n}\right\}$ converges strongly to a minimizer $x^{*}$ of the CMP (1.2), which is a unique solution $x^{*}$ in $\Omega$ to the HVIP

$$
\left\langle(\mu F-\gamma S) x^{*}, p-x^{*}\right\rangle \geq 0, \quad \forall p \in \Omega
$$

In Theorem 3.1, putting $M=N=1$, we obtain the following.

Corollary 3.2 Let $C$ be a nonempty closed convex subset of a real Hilbert space $H$. Let $f: C \rightarrow \mathbf{R}$ be a convex functional with L-Lipschitz-continuous gradient $\nabla f$. Let $\Theta_{1}$ be a bifunction from $C \times C$ to $\mathbf{R}$ satisfying (A1)-(A4), $\varphi_{1}: C \rightarrow \mathbf{R} \cup\{+\infty\}$ be a proper lower semicontinuous and convex function with restriction (B1) or (B2), and $A_{1}: H \rightarrow H$ be $\mu_{1}$ inverse-strongly monotone. Let $R_{1}: C \rightarrow 2^{H}$ be a maximal monotone mapping and $B_{1}$ : $C \rightarrow H$ be $\eta_{1}$-inverse-strongly monotone. Let $T: C \rightarrow C$ be a $\xi$-strictly pseudocontractive mapping, $S: H \rightarrow H$ be a nonexpansive mapping and $V: H \rightarrow H$ be a $\rho$-contraction with coefficient $\rho \in[0,1)$. Let $F: H \rightarrow H$ be $\kappa$-Lipschitzian and $\eta$-strongly monotone with positive constants $\kappa, \eta>0$ such that $0 \leq \gamma<\tau$ and $0<\mu<\frac{2 \eta}{\kappa^{2}}$ where $\tau=1-\sqrt{1-\mu\left(2 \eta-\mu \kappa^{2}\right)}$. Assume that $\Omega:=\operatorname{GMEP}\left(\Theta_{1}, \varphi_{1}, A_{1}\right) \cap \mathrm{I}\left(B_{1}, R_{1}\right) \cap \operatorname{Fix}(T) \cap \Gamma \neq \emptyset$. Let $\left\{\lambda_{n}\right\} \subset[a, b] \subset\left(0, \frac{2}{L}\right)$, $\left\{\alpha_{n}\right\} \subset(0, \infty)$ with $\sum_{n=0}^{\infty} \alpha_{n}<\infty,\left\{\epsilon_{n}\right\},\left\{\delta_{n}\right\},\left\{\beta_{n}\right\},\left\{\gamma_{n}\right\},\left\{\sigma_{n}\right\} \subset(0,1)$ with $\beta_{n}+\gamma_{n}+\sigma_{n}=1$, and $\left\{r_{1, n}\right\} \subset\left[c_{1}, d_{1}\right] \subset\left(0,2 \mu_{1}\right),\left\{\lambda_{1, n}\right\} \subset\left[a_{1}, b_{1}\right] \subset\left(0,2 \eta_{1}\right)$. For arbitrarily given $x_{0} \in H$, let $\left\{x_{n}\right\}$ be a sequence generated by

$$
\left\{\begin{array}{l}
\Theta\left(u_{n}, y\right)+\varphi(y)-\varphi\left(u_{n}\right)+\left\langle A x_{n}, y-u_{n}\right\rangle+\frac{1}{r_{n}}\left\langle y-u_{n}, u_{n}-x_{n}\right\rangle \geq 0, \quad \forall y \in C, \\
v_{n}=J_{R_{1}, \lambda_{1, n}}\left(I-\lambda_{1, n} B_{1}\right) u_{n} \\
y_{n}=\beta_{n} x_{n}+\gamma_{n} P_{C}\left(I-\lambda_{n} \nabla f_{\alpha_{n}}\right) v_{n}+\sigma_{n} T P_{C}\left(I-\lambda_{n} \nabla f_{\alpha_{n}}\right) v_{n}, \\
x_{n+1}=\epsilon_{n} \gamma\left(\delta_{n} V x_{n}+\left(1-\delta_{n}\right) S x_{n}\right)+\left(I-\epsilon_{n} \mu F\right) y_{n}, \quad \forall n \geq 0,
\end{array}\right.
$$

where $\nabla f_{\alpha_{n}}=\alpha_{n} I+\nabla f$ for all $n \geq 0$. Suppose that

(C1) $\lim _{n \rightarrow \infty} \epsilon_{n}=0, \sum_{n=0}^{\infty} \epsilon_{n}=\infty$ and $\lim _{n \rightarrow \infty} \frac{1}{\epsilon_{n}}\left|1-\frac{\delta_{n-1}}{\delta_{n}}\right|=0$;

(C2) $\lim \sup _{n \rightarrow \infty} \frac{\delta_{n}}{\epsilon_{n}}<\infty, \lim _{n \rightarrow \infty} \frac{1}{\epsilon_{n}}\left|\frac{1}{\delta_{n}}-\frac{1}{\delta_{n-1}}\right|=0$ and $\lim _{n \rightarrow \infty} \frac{1}{\delta_{n}}\left|1-\frac{\epsilon_{n-1}}{\epsilon_{n}}\right|=0$;

(C3) $\lim _{n \rightarrow \infty} \frac{\left|\beta_{n}-\beta_{n-1}\right|}{\epsilon_{n} \delta_{n}}=0$ and $\lim _{n \rightarrow \infty} \frac{\left|\gamma_{n}-\gamma_{n-1}\right|}{\epsilon_{n} \delta_{n}}=0$;

(C4) $\lim _{n \rightarrow \infty} \frac{\left|\lambda_{1, n}-\lambda_{1, n-1}\right|}{\epsilon_{n} \delta_{n}}=0$ and $\lim _{n \rightarrow \infty} \frac{\left|r_{1, n}-r_{1, n-1}\right|}{\epsilon_{n} \delta_{n}}=0$;

(C5) $\lim _{n \rightarrow \infty} \frac{\left|\lambda_{n}-\lambda_{n-1}\right|}{\epsilon_{n} \delta_{n}}=0, \lim _{n \rightarrow \infty} \frac{\left|\lambda_{n} \alpha_{n}-\lambda_{n-1} \alpha_{n-1}\right|}{\epsilon_{n} \delta_{n}}=0$ and $\left(\gamma_{n}+\sigma_{n}\right) \xi \leq \gamma_{n}$ for all $n \geq 0$;

(C6) $\left\{\beta_{n}\right\} \subset[c, d] \subset(0,1), \lim _{n \rightarrow \infty} \frac{\alpha_{n}}{\delta_{n}}=0$ and $\liminf _{n \rightarrow \infty} \sigma_{n}>0$.

Then we have:

(i) $\lim _{n \rightarrow \infty} \frac{\left\|x_{n+1}-x_{n}\right\|}{\delta_{n}}=0$;

(ii) $\omega_{w}\left(x_{n}\right) \subset \Omega$;

(iii) $\left\{x_{n}\right\}$ converges strongly to a minimizer $x^{*}$ of the CMP (1.2), which is a unique solution $x^{*}$ in $\Omega$ to the HVIP

$$
\left\langle(\mu F-\gamma S) x^{*}, p-x^{*}\right\rangle \geq 0, \quad \forall p \in \Omega
$$

In Theorem 3.1, putting $M=N=1, \mu=2$, and $F \equiv \frac{1}{2} I$ where $I$ is the identity mapping on $H$, we obtain the following.

Corollary 3.3 Let $C$ be a nonempty closed convex subset of a real Hilbert space $H$. Let $f$ : $C \rightarrow \mathbf{R}$ be a convex functional with L-Lipschitz-continuous gradient $\nabla f$. Let $\Theta_{1}$ be a bifunc- 
tion from $C \times C$ to $\mathbf{R}$ satisfying (A1)-(A4), $\varphi_{1}: C \rightarrow \mathbf{R} \cup\{+\infty\}$ be a proper lower semicontinuous and convex function with restriction (B1) or (B2), and $A_{1}: H \rightarrow H$ be $\mu_{1}$-inversestrongly monotone. Let $R_{1}: C \rightarrow 2^{H}$ be a maximal monotone mapping and $B_{1}: C \rightarrow H$ be $\eta_{1}$-inverse-strongly monotone. Let $T: C \rightarrow C$ be a $\xi$-strictly pseudocontractive mapping, $S: H \rightarrow H$ be a nonexpansive mapping and $V: H \rightarrow H$ be a $\rho$-contraction with coefficient $\rho \in[0,1)$. Assume that $\Omega:=\operatorname{GMEP}\left(\Theta_{1}, \varphi_{1}, A_{1}\right) \cap \mathrm{I}\left(B_{1}, R_{1}\right) \cap \operatorname{Fix}(T) \cap \Gamma \neq \emptyset$. Let $0 \leq \gamma<1$, $\left\{\lambda_{n}\right\} \subset[a, b] \subset\left(0, \frac{2}{L}\right),\left\{\alpha_{n}\right\} \subset(0, \infty)$ with $\sum_{n=0}^{\infty} \alpha_{n}<\infty,\left\{\epsilon_{n}\right\},\left\{\delta_{n}\right\},\left\{\beta_{n}\right\},\left\{\gamma_{n}\right\},\left\{\sigma_{n}\right\} \subset(0,1)$ with $\beta_{n}+\gamma_{n}+\sigma_{n}=1$, and $\left\{r_{1, n}\right\} \subset\left[c_{1}, d_{1}\right] \subset\left(0,2 \mu_{1}\right),\left\{\lambda_{1, n}\right\} \subset\left[a_{1}, b_{1}\right] \subset\left(0,2 \eta_{1}\right)$. For arbitrarily given $x_{0} \in H$, let $\left\{x_{n}\right\}$ be a sequence generated by

$$
\left\{\begin{array}{l}
\Theta\left(u_{n}, y\right)+\varphi(y)-\varphi\left(u_{n}\right)+\left\langle A x_{n}, y-u_{n}\right\rangle+\frac{1}{r_{n}}\left\langle y-u_{n}, u_{n}-x_{n}\right\rangle \geq 0, \quad \forall y \in C, \\
v_{n}=J_{R_{1}, \lambda_{1, n}}\left(I-\lambda_{1, n} B_{1}\right) u_{n} \\
y_{n}=\beta_{n} x_{n}+\gamma_{n} P_{C}\left(I-\lambda_{n} \nabla f_{\alpha_{n}}\right) v_{n}+\sigma_{n} T P_{C}\left(I-\lambda_{n} \nabla f_{\alpha_{n}}\right) v_{n}, \\
x_{n+1}=\epsilon_{n} \gamma\left(\delta_{n} V x_{n}+\left(1-\delta_{n}\right) S x_{n}\right)+\left(1-\epsilon_{n}\right) y_{n}, \quad \forall n \geq 0,
\end{array}\right.
$$

where $\nabla f_{\alpha_{n}}=\alpha_{n} I+\nabla f$ for all $n \geq 0$. Suppose that

(C1) $\lim _{n \rightarrow \infty} \epsilon_{n}=0, \sum_{n=0}^{\infty} \epsilon_{n}=\infty$ and $\lim _{n \rightarrow \infty} \frac{1}{\epsilon_{n}}\left|1-\frac{\delta_{n-1}}{\delta_{n}}\right|=0$;

(C2) $\lim \sup _{n \rightarrow \infty} \frac{\delta_{n}}{\epsilon_{n}}<\infty, \lim _{n \rightarrow \infty} \frac{1}{\epsilon_{n}}\left|\frac{1}{\delta_{n}}-\frac{1}{\delta_{n-1}}\right|=0$ and $\lim _{n \rightarrow \infty} \frac{1}{\delta_{n}}\left|1-\frac{\epsilon_{n-1}}{\epsilon_{n}}\right|=0$;

(C3) $\lim _{n \rightarrow \infty} \frac{\left|\beta_{n}-\beta_{n-1}\right|}{\epsilon_{n} \delta_{n}}=0$ and $\lim _{n \rightarrow \infty} \frac{\left|\gamma_{n}-\gamma_{n-1}\right|}{\epsilon_{n} \delta_{n}}=0$;

(C4) $\lim _{n \rightarrow \infty} \frac{\left|\lambda_{1, n}-\lambda_{1, n-1}\right|}{\epsilon_{n} \delta_{n}}=0$ and $\lim _{n \rightarrow \infty} \frac{\left|n_{1, n}-r_{1, n-1}\right|}{\epsilon_{n} \delta_{n}}=0$;

(C5) $\lim _{n \rightarrow \infty} \frac{\left|\lambda_{n}-\lambda_{n-1}\right|}{\epsilon_{n} \delta_{n}}=0, \lim _{n \rightarrow \infty} \frac{\left|\lambda_{n} \alpha_{n}-\lambda_{n-1} \alpha_{n-1}\right|}{\epsilon_{n} \delta_{n}}=0$ and $\left(\gamma_{n}+\sigma_{n}\right) \xi \leq \gamma_{n}$ for all $n \geq 0$;

(C6) $\left\{\beta_{n}\right\} \subset[c, d] \subset(0,1), \lim _{n \rightarrow \infty} \frac{\alpha_{n}}{\delta_{n}}=0$ and $\liminf _{n \rightarrow \infty} \sigma_{n}>0$.

Then we have:

(i) $\lim _{n \rightarrow \infty} \frac{\left\|x_{n+1}-x_{n}\right\|}{\delta_{n}}=0$;

(ii) $\omega_{w}\left(x_{n}\right) \subset \Omega$;

(iii) $\left\{x_{n}\right\}$ converges strongly to a minimizer $x^{*}$ of the CMP (1.2), which is a unique solution $x^{*}$ in $\Omega$ to the HVIP

$$
\left\langle(I-\gamma S) x^{*}, p-x^{*}\right\rangle \geq 0, \quad \forall p \in \Omega .
$$

Remark 3.1 It is obvious that our iterative scheme (3.1) is very different from Xu's iterative one (1.4) and Yao et al.'s iterative one (1.8). Here, Xu's iterative scheme in [20, Theorem 5.2] is extended to develop our four-step iterative scheme (3.1) for the CMP (1.2) with constraints of finite many GMEPs, finite many variational inclusions and the fixed point problem of a strict pseudocontraction by combining Korpelevich's extragradient method, the viscosity approximation method, the hybrid steepest-descent method, Mann's iteration method, and the gradient-projection method (GPM) with regularization. It is worth pointing out that under the lack of the assumptions similar to those in [21, Theorem 3.2], e.g., $\left\{x_{n}\right\}$ is bounded, $\operatorname{Fix}(T) \cap \operatorname{int} C \neq \emptyset$ and $\|x-T x\| \geq k \operatorname{Dist}(x, \operatorname{Fix}(T))$, $\forall x \in C$ for some $k>0$, the sequence $\left\{x_{n}\right\}$ generated by (3.1) converges strongly to a point $x^{*} \in \bigcap_{k=1}^{M} \operatorname{GMEP}\left(\Theta_{k}, \varphi_{k}, A_{k}\right) \cap \bigcap_{i=1}^{N} \mathrm{I}\left(B_{i}, R_{i}\right) \cap \operatorname{Fix}(T) \cap \Gamma=: \Omega$, which is a unique solution of the HVIP (over the fixed point set of strictly pseudocontractive mapping $T$ ), i.e., $\left\langle(\mu F-\gamma S) x^{*}, p-x^{*}\right\rangle \geq 0, \forall p \in \Omega$.

Remark 3.2 Our Theorem 3.1 improves, extends, supplements, and develops Xu [20, Theorem 5.2] and Yao et al. [21, Theorems 3.1 and 3.2] in the following aspects: 
(a) Our HVIP with the unique solution $x^{*} \in \Omega$ satisfying

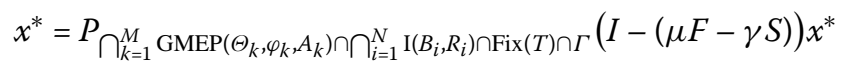

is more general than the problem of finding a point $\tilde{x} \in C$ satisfying $\tilde{x}=P_{\operatorname{Fix}(T)} S \tilde{x}$ in [21] and very different from the problem of finding a point $x^{*} \in \Gamma$ satisfying $x^{*}=P_{\Gamma} V x^{*}$ in [20, Theorem 5.2]. It is worth emphasizing that the nonexpansive mapping $T$ in [21, Theorems 3.1 and 3.2] is extended to the strict pseudocontraction $T$ and the CMP in [20, Theorem 5.2] is generalized to the setting of finitely many GMEPs, finitely many variational inclusions and the fixed point problem of a strict pseudocontraction $T$.

(b) Our four-step iterative scheme (3.1) for the CMP with constraints of several problems is more flexible, more advantageous and more subtle than Xu's one-step iterative one (1.4) and than Yao et al.'s two-step iterative one (1.8) because it can be used to solve two kinds of problems, e.g., the HVIP (over the fixed point set of strictly pseudocontractive mapping $T$ ), and the problem of finding a common point of four sets: $\bigcap_{k=1}^{M} \operatorname{GMEP}\left(\Theta_{k}, \varphi_{k}, A_{k}\right)$, $\bigcap_{i=1}^{N} \mathrm{I}\left(B_{i}, R_{i}\right)$, Fix $(T)$ and $\Gamma$. In addition, our Theorem 3.1 also drops the crucial requirements in [21, Theorem 3.2(v)] that $\operatorname{Fix}(T) \cap \operatorname{int} C \neq \emptyset$ and $\|x-T x\| \geq k \operatorname{Dist}(x, \operatorname{Fix}(T))$, $\forall x \in C$ for some $k>0$.

(c) The techniques for the argument in our Theorem 3.1 are very different from [21, Theorems 3.1 and 3.2] and from [20, Theorem 5.2] because we make use of the properties of strictly pseudocontractive mappings (see Lemmas 2.3 and 2.4), the ones of resolvent operators and maximal monotone mappings (see Proposition 2.2, Remark 2.2, and Lemmas 2.9-2.13), the ones of averaged mappings (see Propositions 2.3 and 2.4), the inclusion problem $0 \in \widetilde{T} v(\Leftrightarrow v \in \operatorname{VI}(C, A)$ for maximal monotone operator $\widetilde{T}$ ) (see (2.2)), and the contractive coefficient estimates for the contractions associated with nonexpansive mappings (see Lemma 2.7).

(d) Compared with the restrictions on the parameter sequences of [21, Theorem 3.2] and [20, Theorem 5.2], respectively, the hypotheses (C3)-(C6) in our Theorem 3.1 are added because our Theorem 3.1 involves the quite complex problem, i.e., the HVIP (over the fixed point set of strictly pseudocontractive mapping $T$ ) with constraints of several problems: CMP (1.2), finitely many GMEPs and finitely many variational inclusions.

Competing interests
The authors declare that they have no competing interests.

Authors' contributions

All authors read and approved the final manuscript.

\footnotetext{
Author details

${ }^{1}$ Department of Mathematics, Shanghai Normal University, Shanghai, 200234, China. ${ }^{2}$ Scientific Computing Key Laboratory of Shanghai Universities, Shanghai, 200234, China. ${ }^{3}$ Department of Information Management, Cheng Shiu University, Kaohsiung, 833, Taiwan. ${ }^{4}$ Center for Fundamental Science, Kaohsiung Medical University, Kaohsiung, 807, Taiwan.
}

\section{Acknowledgements}

LC Ceng was partially supported by the National Science Foundation of China (11071169), Innovation Program of Shanghai Municipal Education Commission (09ZZ133) and PhD Program Foundation of Ministry of Education of China (20123127110002). YC Liou was supported in part by the Grants NSC 101-2628-E-230-001-MY3 and NSC

103-2923-E-037-001-MY3. CF Wen was partially supported by the Grant NSC 103-2923-E-037-001-MY3. 


\section{References}

1. Lions, JL: Quelques Méthodes de Résolution des Problèmes aux Limites Non Linéaires. Dunod, Paris (1969)

2. Glowinski, R: Numerical Methods for Nonlinear Variational Problems. Springer, New York (1984)

3. Takahashi, W: Nonlinear Functional Analysis. Yokohama Publishers, Yokohama (2000)

4. Oden, JT: Quantitative Methods on Nonlinear Mechanics. Prentice Hall, Englewood Cliffs (1986)

5. Zeidler, E: Nonlinear Functional Analysis and Its Applications. Springer, New York (1985)

6. Korpelevich, GM: The extragradient method for finding saddle points and other problems. Matecon 12, 747-756 (1976)

7. Cai, G, Bu, SQ: Strong and weak convergence theorems for general mixed equilibrium problems and variational inequality problems and fixed point problems in Hilbert spaces. J. Comput. Appl. Math. 247, 34-52 (2013)

8. Zeng, LC, Yao, JC: Strong convergence theorem by an extragradient method for fixed point problems and variational inequality problems. Taiwan. J. Math. 10(5), 1293-1303 (2006)

9. Peng, JW, Yao, JC: A new hybrid-extragradient method for generalized mixed equilibrium problems, fixed point problems and variational inequality problems. Taiwan. J. Math. 12, 1401-1432 (2008)

10. Ceng, LC, Ansari, QH, Schaible, S: Hybrid extragradient-like methods for generalized mixed equilibrium problems, system of generalized equilibrium problems and optimization problems. J. Glob. Optim. 53, $69-96$ (2012)

11. Yao, Y, Liou, YC, Kang, SM: Approach to common elements of variational inequality problems and fixed point problems via a relaxed extragradient method. Comput. Math. Appl. 59, 3472-3480 (2010)

12. Ceng, LC, Ansari, QH, Wong, MM, Yao, JC: Mann type hybrid extragradient method for variational inequalities, variational inclusions and fixed point problems. Fixed Point Theory 13(2), 403-422 (2012)

13. Nadezhkina, N, Takahashi, W: Weak convergence theorem by an extragradient method for nonexpansive mappings and monotone mappings. J. Optim. Theory Appl. 128, 191-201 (2006)

14. Ceng, LC, Hadjisavvas, N, Wong, NC: Strong convergence theorem by a hybrid extragradient-like approximation method for variational inequalities and fixed point problems. J. Glob. Optim. 46, 635-646 (2010)

15. Ceng, LC, Yao, JC: A relaxed extragradient-like method for a generalized mixed equilibrium problem, a general system of generalized equilibria and a fixed point problem. Nonlinear Anal. 72, 1922-1937 (2010)

16. Ceng, LC, Wong, MM: Relaxed extragradient method for finding a common element of systems of variational inequalities and fixed point problems. Taiwan. J. Math. 17(2), $701-724$ (2013)

17. Ceng, LC, Liao, CW, Pang, CT, Wen, CF: Convex minimization with constraints of systems of variational inequalities, mixed equilibrium, variational inequality, and fixed point problems. J. Appl. Math. 2014, Article ID 105928 (2014)

18. Ceng, LC, Ansari, QH, Yao, JC: Relaxed extragradient iterative methods for variational inequalities. Appl. Math. Comput. 218, 1112-1123 (2011)

19. Ceng, LC, Petrusel, A: Relaxed extragradient-like method for general system of generalized mixed equilibria and fixed point problem. Taiwan. J. Math. 16(2), 445-478 (2012)

20. Xu, HK: Averaged mappings and the gradient-projection algorithm. J. Optim. Theory Appl. 150, 360-378 (2011)

21. Yao, Y, Liou, YC, Marino, G: Two-step iterative algorithms for hierarchical fixed point problems and variational inequality problems. J. Appl. Math. Comput. 31(1-2), 433-445 (2009)

22. Ceng, LC, Guu, SM, Yao, JC: Hybrid iterative method for finding common solutions of generalized mixed equilibrium and fixed point problems. Fixed Point Theory Appl. 2012, 92 (2012)

23. Ceng, $\mathrm{LC}, \mathrm{Hu}, \mathrm{HY}$, Wong, $\mathrm{MM}$ : Strong and weak convergence theorems for generalized mixed equilibrium problem with perturbation and fixed point problem of infinitely many nonexpansive mappings. Taiwan. J. Math. 15(3), 1341-1367 (2011)

24. Ceng, LC, Al-Homidan, S: Algorithms of common solutions for generalized mixed equilibria, variational inclusions, and constrained convex minimization. Abstr. Appl. Anal. 2014, Article ID 132053 (2014)

25. Takahashi, S, Takahashi, W: Strong convergence theorem for a generalized equilibrium problem and a nonexpansive mapping in a Hilbert space. Nonlinear Anal. 69, 1025-1033 (2008)

26. Ceng, LC, Yao, JC: A hybrid iterative scheme for mixed equilibrium problems and fixed point problems. J. Comput. Appl. Math. 214, 186-201 (2008)

27. Colao, V, Marino, G, Xu, HK: An iterative method for finding common solutions of equilibrium and fixed point problems. J. Math. Anal. Appl. 344, 340-352 (2008)

28. Ceng, LC, Petrusel, A, Yao, JC: Iterative approaches to solving equilibrium problems and fixed point problems of infinitely many nonexpansive mappings. J. Optim. Theory Appl. 143, 37-58 (2009)

29. Rockafellar, RT: Monotone operators and the proximal point algorithms. SIAM J. Control Optim. 14, 877-898 (1976)

30. Huang, NJ: A new completely general class of variational inclusions with noncompact valued mappings. Comput. Math. Appl. 35(10), 9-14 (1998)

31. Zeng, LC, Guu, SM, Yao, JC: Characterization of $\mathrm{H}$-monotone operators with applications to variational inclusions. Comput. Math. Appl. 50(3-4), 329-337 (2005)

32. Ceng, LC, Guu, SM, Yao, JC: Iterative approximation of solutions for a class of completely generalized set-valued quasi-variational inclusions. Comput. Math. Appl. 56, 978-987 (2008)

33. Zhang, SS, Lee, HWJ, Chan, CK: Algorithms of common solutions for quasi-variational inclusions and fixed point problems. Appl. Math. Mech. 29, 571-581 (2008)

34. Ceng, LC, Guu, SM, Yao, JC: Iterative algorithm for finding approximate solutions of mixed quasi-variational-like inclusions. Comput. Math. Appl. 56, 942-952 (2008)

35. Han, D, Lo, HK: Solving non-additive traffic assignment problems: a descent method for co-coercive variational inequalities. Eur. J. Oper. Res. 159, 529-544 (2004)

36. Byrne, C: A unified treatment of some iterative algorithms in signal processing and image reconstruction. Inverse Probl. 20, 103-120 (2004)

37. Combettes, $\mathrm{PL}$ : Solving monotone inclusions via compositions of nonexpansive averaged operators. Optimization 53(5-6), 475-504 (2004)

38. Marino, G, Xu, HK: Weak and strong convergence theorems for strict pseudo-contractions in Hilbert spaces. J. Math. Anal. Appl. 329, 336-346 (2007)

39. Goebel, K, Kirk, WA: Topics on Metric Fixed Point Theory. Cambridge University Press, Cambridge (1990) 
40. $\mathrm{Xu}, \mathrm{HK}, \mathrm{Kim}, \mathrm{TH}$ : Convergence of hybrid steepest-descent methods for variational inequalities. J. Optim. Theory Appl. $119(1), 185-201(2003)$

41. Xu, HK: Iterative algorithms for nonlinear operators. J. Lond. Math. Soc. (2) 66(1), 240-256 (2002)

42. Barbu, V: Nonlinear Semigroups and Differential Equations in Banach Spaces. Noordhoff, Groningen (1976)

43. Yamada, I: The hybrid steepest-descent method for the variational inequality problems over the intersection of the fixed-point sets of nonexpansive mappings. In: Batnariu, D, Censor, Y, Reich, S (eds.) Inherently Parallel Algorithms in Feasibility and Optimization and Their Applications, pp. 473-504. North-Holland, Amsterdam (2001)

44. Baillon, JB, Haddad, G: Quelques propriétés des opérateurs angle-bornés et $n$-cycliquement monotones. Isr. J. Math. $26,137-150(1977)$

10.1186/1029-242X-2014-444

Cite this article as: Ceng et al.: Extragradient method for convex minimization problem. Journal of Inequalities and Applications 2014, 2014:444

\section{Submit your manuscript to a SpringerOpen ${ }^{\circ}$ journal and benefit from:}

- Convenient online submission

- Rigorous peer review

- Immediate publication on acceptance

- Open access: articles freely available online

- High visibility within the field

- Retaining the copyright to your article 